\title{
Fluorescence correlation spectroscopy for studying intermediate filament assembly
}

\author{
Dissertation \\ for the award of the degree \\ "Doctor rerum naturalium" \\ of the Georg-August-Universität Göttingen
}

within the doctoral program

Göttingen Graduate School for Neurosciences, Biophysics, and Molecular Biosciences (GGNB) of the Georg-August University School of Science (GAUSS)

submitted by

Viktor Schroeder

from Rinteln

Göttingen 2017 


\section{Thesis Committee}

Prof. Dr. Sarah Köster

Institute for X-Ray Physics

Georg-August-University Göttingen

Prof. Dr. Dr. Detlev Schild

Department of Neurophysiology \& Cellular Biophysics

Georg-August-University Göttingen

Dr. Florian Rehfeldt

Third Institute of Physics

Georg-August-University Göttingen 


\section{Members of the Examination Board}

Referee: Prof. Dr. Sarah Köster

Institute for X-Ray Physics

Georg-August-University Göttingen

Second Referee: Dr. Florian Rehfeldt

Third Institute of Physics

Georg-August-University Göttingen

\section{Further members of the examination board}

Dr. Thomas Burg

Department for Micro- and Nanotechnology

Max Planck Institute for Biophysical Chemistry Göttingen

Prof. Dr. Jörg Enderlein

Third Institute of Physics

Georg-August-University Göttingen

Prof. Dr. Helmut Grubmüller

Department for Theoretical and Computational Biophysics

Max Planck Institute for Biophysical Chemistry Göttingen

Prof. Dr. Dr. Detlev Schild

Department of Neurophysiology \& Cellular Biophysics

Georg-August-University Göttingen

\section{Date of oral examination}

August $4^{\text {th }}, 2017$ 


\section{Abstract}

Intermediate filaments play a central role in the cytoskeleton of eukaryotic cells. Together with microtubules and actin filaments they determine the mechanical properties of cells. Microtubules are also the guiding tracks for molecular transport in cells while actin filaments play an essential role in cell motility. The diameter of intermediate filaments lies between the diameter of actin filaments and microtubules which led to their name. There is an enormous genetic variety of intermediate filaments. In humans there are over 70 different genes for intermediate filament proteins. The different types of intermediate filaments are celltype-specific. For example, the intermediate filament protein vimentin is found in cells of mesenchymal origin and keratins occur in epithelial cells. Mutations in genes coding for intermediate filaments are known to cause more than 80 diseases, among them Alexander disease and amyotrophic lateral sclerosis (ALS). Intermediate filaments share a common hierarchical assembly scheme. Several tetramers form unit length filaments (ULFs). The ULFs then anneal longitudinally to form elongated filaments.

Studies of the assembly are necessary to understand the structural properties of cells. In this thesis we aim to further understand the assembly of intermediate filaments. We use fluorescence correlation spectroscopy (FCS) which is a versatile technique to study the motility of molecules in solution. During the thesis project, we built a setup suitable for FCS. It allows for detecting the diffusive properties of the different states during the assembly. With the setup we study both the early and the long time scales of the assembly of vimentin filaments. For the long times we employ bulk measurements. To access the early time scales of the assembly, we employ microfluidic techniques. With the microfluidic mixing device we map the temporal axis to a spatial axis. This gives us the possibility to control the interaction of the molecules in a defined manner. We aim to observe the assembly reaction of vimentin intermediate filaments upon the addition of monovalent salt ions. 


\section{Contents}

\begin{tabular}{lll}
\hline 1 & Introduction & 1
\end{tabular}

2 State of the art and theoretical background 3

2.1 Cytoskeletal proteins . . . . . . . . . . . . . . . . 3

2.2 Intermediate filaments $\ldots \ldots \ldots \ldots$. . . . . . . . . . . . . 3

2.2 .1 Vimentin . . . . . . . . . . . . . . . . . . . 4

2.3 Microfluidic techniques . . . . . . . . . . . . . . . . 6

2.3 .1 Viscous flow . . . . . . . . . . . . . . . . . . 6

2.3 .2 Diffusive mixing . . . . . . . . . . . . . . . . . 7

2.3 .3 Microfluidic mixers . . . . . . . . . . . . . . . . . 8

2.4 Fluorescence correlation spectroscopy . . . . . . . . . . . . . . . . . 11

2.4 .1 Fluorescence . . . . . . . . . . . . . . . . . . . . . . . . . . . 11

2.4 .2 Theory of fluorescence correlation spectroscopy . . . . . . . . . . 13

$2.4 .3 \quad$ Further aspects of FCS . . . . . . . . . . . . . . 16

3 Materials and methods $\quad 19$

3.1 Preparation of vimentin $\ldots \ldots \ldots$. . . . . . . . . . . . . . . . . . . . . . 19

$3.1 .1 \quad$ Fluorescent labeling of vimentin . . . . . . . . . . . . . . . . . . . 19

3.1 .2 Label distribution . . . . . . . . . . . . . . . . . . . . . 20

3.1 .3 Dialysis and assembly . . . . . . . . . . . . . . . . . . 20

3.2 Numerical simulations . . . . . . . . . . . . . . . . . . . . . . . . 21

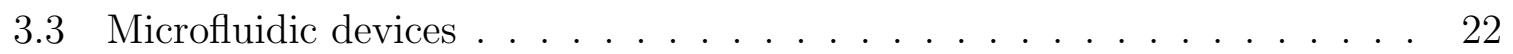

3.3.1 Photo lithography . . . . . . . . . . . . . . . . . . . . . . 22

3.3 .2 Soft lithography . . . . . . . . . . . . . . . . . . . . . . . 23

3.3 .3 Experimental setup . . . . . . . . . . . . . . . . . . 27

3.3 .4 Surface modifications . . . . . . . . . . . . . . . . . . . 27

3.4 Microscopy techniques . . . . . . . . . . . . . . . . . . . . . . . . 28 
\begin{tabular}{lll}
\hline 4 & Results & 31
\end{tabular}

4.1 Construction of the FCS setup . . . . . . . . . . . . . . . . . . 31

$4.1 .1 \quad$ Hardware setup . . . . . . . . . . . . . . . . . . . . . . 31

4.1 .2 Calibration of the setup . . . . . . . . . . . . . 36

4.2 Vimentin labeling . . . . . . . . . . . . . . . . . . . . . . . . . . 36

$4.2 .1 \quad$ Detection of free dye . . . . . . . . . . . . . . . . . . . 38

4.2 .2 Separation of labeled vimentin and free dye . . . . . . . . . . . . 40

4.3 Vimentin assembly in bulk . . . . . . . . . . . . . . . . . . . . . . . . . 41

$4.4 \quad$ Microfluidic device for protein assembly . . . . . . . . . . . . . . . . . . 51

4.4 .1 Criteria for microfluidic mixing . . . . . . . . . . . . . . . 51

4.4 .2 Simulations of microfluidic flow . . . . . . . . . . . . 56

$4.4 .3 \quad$ Fabrication of the final mixing device . . . . . . . . . . . . . . . . 61

4.5 Vimentin assembly in microfluidic flow . . . . . . . . . . . . . . . 63

4.5 .1 Unassembled vimentin in flow . . . . . . . . . . . . . . . . . 63

4.5 .2 Vimentin assembly in flow . . . . . . . . . . . . . . . . . . . . 67

$\begin{array}{llr}5 & \text { Discussion } & 69\end{array}$

$5.1 \quad$ FCS setup . . . . . . . . . . . . . . . . . . . . . . . . . 69

5.2 Vimentin labeling . . . . . . . . . . . . . . . . . . . . . . . . . . . . . . 69

5.3 Vimentin assembly in bulk . . . . . . . . . . . . . . . . . . . . . 70

5.4 Microfluidic device for protein assembly . . . . . . . . . . . . . . . . 71

5.5 Vimentin assembly in microfluidic flow . . . . . . . . . . . . . . 71

$\begin{array}{lll}6 & \text { Conclusions and Outlook } & 73\end{array}$

\begin{tabular}{lll}
\hline A & Data acquisition script & 77
\end{tabular} 


\section{List of Figures}

2.1 Sketch of vimentin assembly states . . . . . . . . . . . . . . . 4

2.2 Atomic model of the vimentin dimer f . . . . . . . . . . . . . . . 5

2.3 Sketch of a five-inlet mixing device . . . . . . . . . . . . . . . . . . . 9

2.4 Sketch of a three-inlet device with a bottleneck structure . . . . . . . . . 10

2.5 Diffusion coefficients of vimentin filaments . . . . . . . . . . . . . . . . . 11

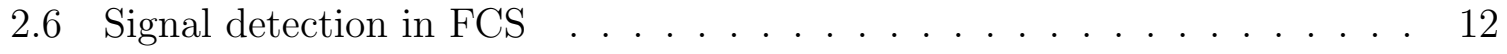

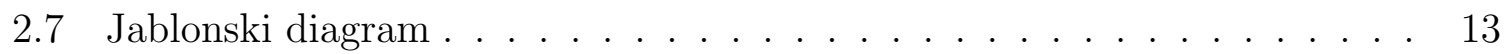

$3.1 \quad$ Sketch of the five-inlet device with constraining step . . . . . . . . . . . . 21

3.2 Photolithography steps for the fabrication of master wafer . . . . . . . . 22

3.3 Cross-section of PDMS channels . . . . . . . . . . . . . . . . . . . . . . . 24

3.4 Molding of step structure . . . . . . . . . . . . . . . . . . . . . . 25

3.5 Image of a molded step structure . . . . . . . . . . . . . . . . 25

3.6 Images of the microfluidic devices . . . . . . . . . . . . . . . 26

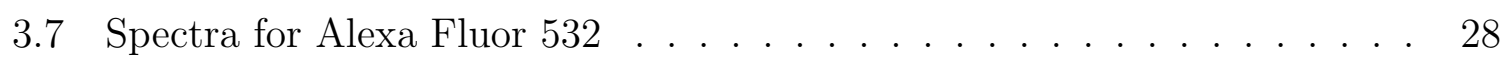

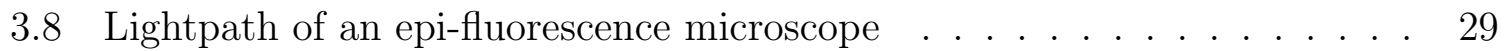

4.1 Beam path of the FCS setup . . . . . . . . . . . . . . . . . . . . 32

$4.2 \quad$ Spectra of ATTO532 and the fluorescence filters . . . . . . . . . . . . 33

4.3 Images of the FCS setup . . . . . . . . . . . . . . . . . . . . . 35

4.4 FCS curve for Rhodamine 6G $\ldots \ldots \ldots$. . . . . . . . . . . . . . 37

4.5 Fluorescence image of assembled vimentin filaments . . . . . . . . . . . . 37

$4.6 \quad$ FCS curve for Atto $532 \ldots \ldots \ldots \ldots$

$4.7 \quad$ FCS measurement of vimentin labeled with Atto $532 \quad \ldots \ldots$

4.8 Unassembled vimentin measured in continuous mode . . . . . . . . . . . 42

4.9 Vimentin assembly measured in continuous mode . . . . . . . . . . . . . 44

4.10 Diffusion coefficient of vimentin diluted after one minute of assembly . . 45 
List of Figures

4.11 Overlay of FCS curves for vimentin assembly . . . . . . . . . . . . . . . . 46

4.12 Vimentin assembly in the continuous mode and in the reaction tube mode 47

4.13 Dependence between storage time after dialysis and the ensuing vimentin assembly . . . . . . . . . . . . . . . . . . . . . . . . . . 48

4.14 Salt dependence of vimentin assembly . . . . . . . . . . . . . . . . . . 49

4.15 Temperature dependence of vimentin assembly . . . . . . . . . . . . . 50

4.16 Five-inlet mixing device . . . . . . . . . . . . . . . . 51

4.17 Focus cone in microfluidic channel . . . . . . . . . . . . . . . . . . . 53

4.18 Influence of flow on the autocorrelation curves . . . . . . . . . . . . . 55

4.19 Comparison of the simulations for devices with and without the constraining step . . . . . . . . . . . . . . . . . 58

4.20 Results of the vimentin flow simulation . . . . . . . . . . . . . . . . . . . 59

4.21 Simulation of protein and salt concentration along the channel . . . . . . 60

4.22 Five-inlet mixing device with constraining step . . . . . . . . . . . . . 61

4.23 Comparison of simulation and experiment for step structure . . . . . . . 62

4.24 Scan of the mixing area . . . . . . . . . . . . . . . . . . . . . . . . . 64

4.25 FCS scan of vimentin stream without assembly trigger at $2 \mathrm{~mm}$ downstream. 66

4.26 Comparison of vimentin at $5 \mathrm{~mm}$ and $10 \mathrm{~mm}$. . . . . . . . . . . 68 


\section{List of Tables}

2.1 Types of intermediate filaments . . . . . . . . . . . . . 4

$3.1 \quad$ Fluorescent dyes used in this thesis project. The molecular weight is given for the dyes with a maleimide group. . . . . . . . . . . . . . . . . . 20

3.2 Labeling ratios and expected number of labeled monomers in a tetramer 20

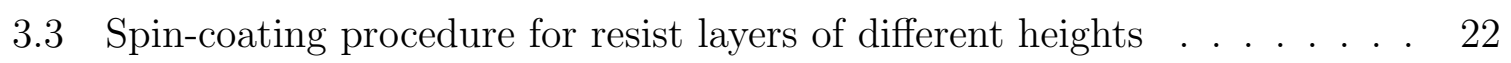

4.1 Estimation of the flow speed for FCS measurements . . . . . . . . . . . . 55 



\section{Introduction}

Intermediate filaments play a central role in the cytoskeleton of eukaryotic cells. Together with microtubules and actin filaments they determine the mechanical properties of cells. Microtubules are also the guiding tracks for molecular transport in cells while actin filaments play an essential role in cell motility. The diameter of intermediate filaments lies between the diameter of actin filaments and microtubules which led to their name. There is an enormous genetic variety of intermediate filaments. In humans there are over 70 different genes for intermediate filament proteins [1]. The different types of intermediate filaments are celltype-specific. For example, the intermediate filament protein vimentin is found in cells of mesenchymal origin and keratins occur in epithelial cells. Mutations in genes coding for intermediate filaments are known to cause more than 80 diseases, among them Alexander disease and amyotrophic lateral sclerosis (ALS) [2].

Studies of the cytoskeleton in vivo are difficult to perform, since the complexity of the cytoskeleton makes it hard identify the role of single molecules. By investigating the structure and function of individual components separately, a whole picture can be obtained.

In this thesis we aim to further understand the assembly of intermediate filaments. Studies of the assembly are necessary to understand the structural properties of cells. We use fluorescence correlation spectroscopy (FCS) which is a versatile technique to study the motility of molecules in solution. The technique takes advantage of the characteristic diffusive properties of molecules with different sizes. It allows for detecting the diffusive properties of the varying states during the assembly. To access the early time scales of the assembly we employ microfluidic techniques. With the microfluidic mixing device we map the temporal axis to a spatial axis. This gives us the possibility to control the interaction of the molecules in a defined manner. With the microfluidic device we aim to study the assembly of intermediate filaments.

We will give an overview on intermediate filaments and the relevant aspects of microfluidics and FCS in Chapter 2. In Chapter 3 we will show the materials and methods 


\section{Introduction}

used for the experiments in this thesis. The FCS setup that was built during this thesis project, the microfluidic techniques and the experiments on vimentin assembly are presented in Chapter 4. Also, the numerical simulations of the microfluidic mixing device are presented in this chapter. Afterwards, we will discuss the results of the thesis in context with the literature in Chapter 5 and give conclusions and outlooks in Chapter 6 . 


\section{State of the art and theoretical background}

\subsection{Cytoskeletal proteins}

The cytoskeleton is responsible for the mechanical integrity of the cell. In metazoan cells, it consists of three main filament components which are microtubules, actin filaments, and intermediate filaments. Microtubules (MTs) function as tracks for kinesin molecular motors. During cell division MTs build up the mitotic spindle that is responsible for separating the chromosomes. Micro-filaments are the second major component. They consist of actin. Cells move and contract by myosin molecules interacting with actin filaments [3]. Both micro-filaments and microtubules are polar structures. Motor proteins move along these filaments in a specific direction.

\subsection{Intermediate filaments}

Intermediate filaments (IFs) are one type of cytoskeletal filaments. Intermediate filaments lack a directionality and no associated motor proteins are known. The IF are grouped into five categories [4]. (see Table 2.1). While keratins are found in epithelial cells, vimentin is found in cells of mesenchymal origin. All intermediate filaments share a similar secondary structure with $\alpha$-helical 'rod' domain and unstructured 'head' and 'tail' domains. The assembly mechanism is similar for all intermediate filaments. The assembly mechanism will be described in detail below. The various IF proteins exhibit varying assembly speeds. For example, keratin K8/K18 assembles at the same speed as vimentin at a 100 times higher concentration [5].

Desmin and vimentin show a compaction step during assembly that is not found in keratins [6]. The diameter of vimentin changes from $17 \mathrm{~nm}$ to $11 \mathrm{~nm}$ during this 
Table 2.1: Types of intermediate filaments. IF proteins are grouped into five categories.

\begin{tabular}{ll}
\hline Type I & Acidic keratins \\
Type II & Basic keratins \\
Type III & Desmin, GFAP, vimentin \\
Type IV & Neurofilaments \\
Type V & Nuclear lamins \\
\hline
\end{tabular}
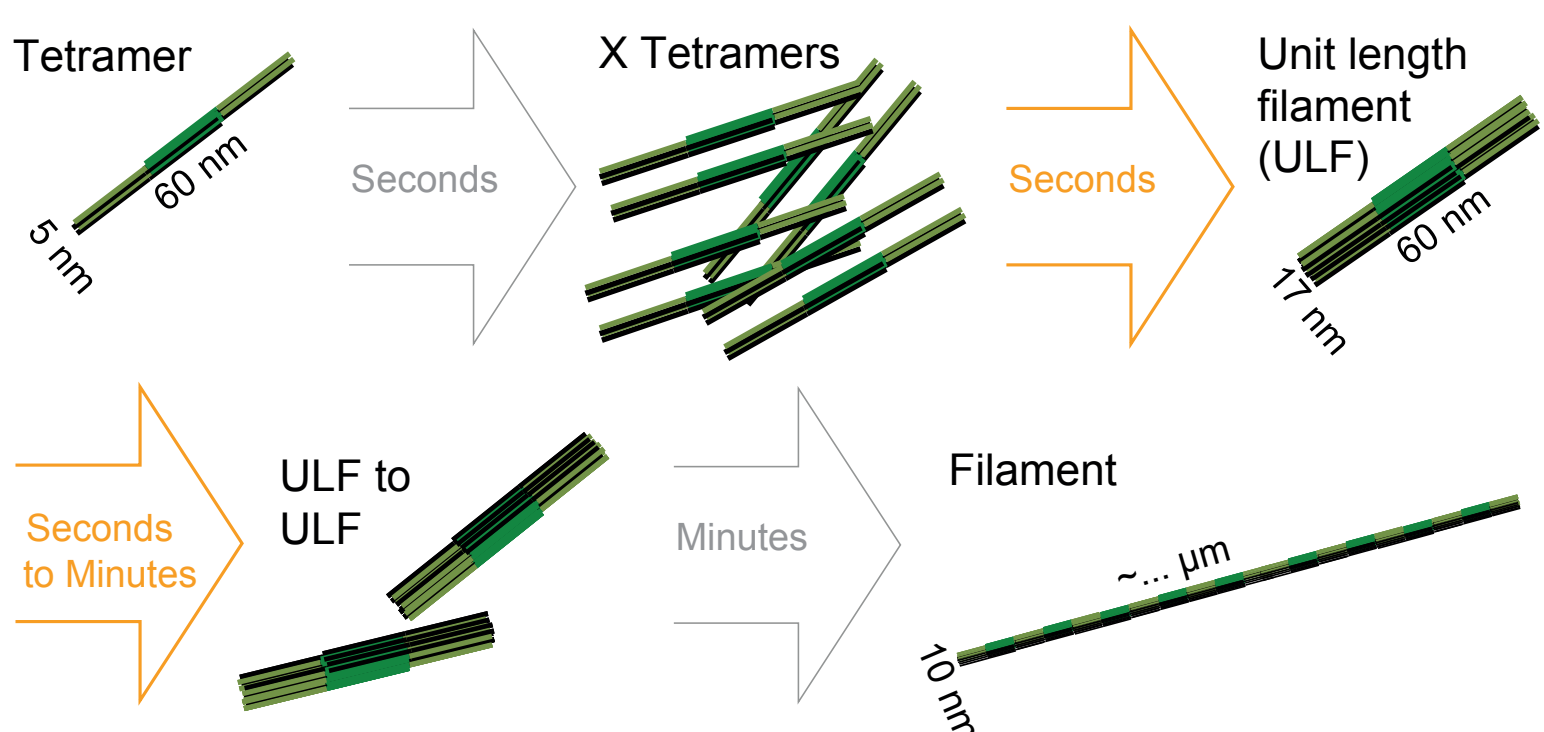

Filament

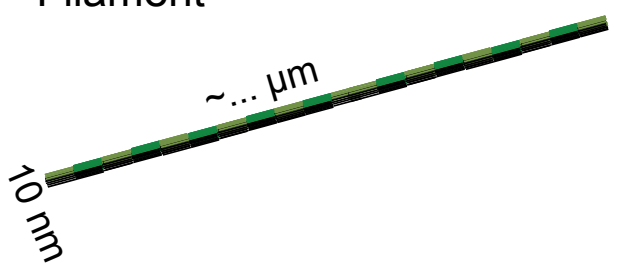

Figure 2.1: Sketch of vimentin assembly states. The assembly is initiated by the addition of monovalent ions. Tetramers align laterally to form unit-length filaments. Full filaments are formed by the end-to-end annealing of unitlength filaments.

compaction step. Mutations in genes coding for IFs are known to cause more than 80 diseases, among them Alexander disease and amyotrophic lateral sclerosis (ALS) [2]. A mutation in the vimentin gene is associated with a dominant, pulverulent cataract [7].

\subsubsection{Vimentin}

Vimentin is found in cells of mesenchymal origin like fibroblasts or endothelial cells [8]. The assembly into filaments takes place in a hierarchical manner. Two vimentin monomers dimerize into a coiled-coil structure (see Figure 2.2). Tetramers form by the alignment of two dimers in an anti-parallel way. Around eight tetramers then assemble 


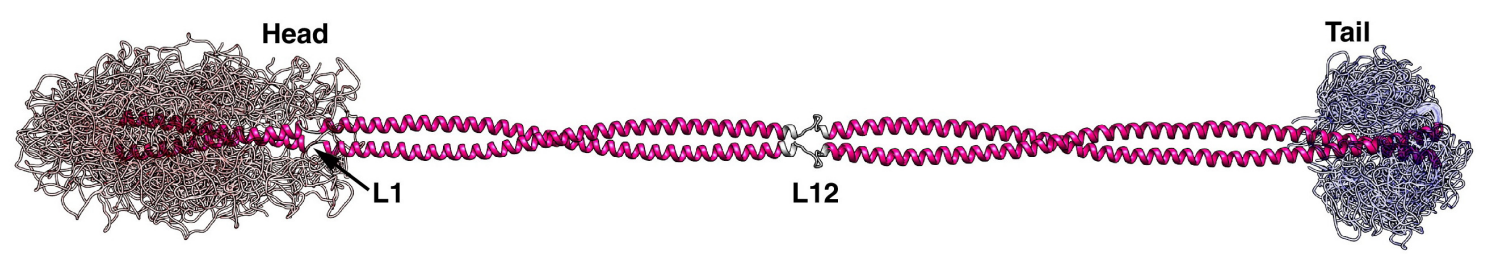

Figure 2.2: Atomic model of the vimentin dimer. The 'rod' domain is separated by the linkers L1 and L12 into three coils. The structure was found by computational modeling. The unordered head and tail domains are represented each by a cloud of 40 possible configurations. Figure taken with permission from [13].

into a unit-length filament (ULF) (see Figure 2.1). The number of tetramers depends on the buffer conditions. ULFs have a length of around $60 \mathrm{~nm}$ and a diameter of $17 \mathrm{~nm}$ [9]. Elongated filaments form by longitudinal annealing of ULFs. The filaments are non-polar because of the anti-parallelity of the tetramer. It was shown that assembled vimentin exchanges subunits with a pool of tetramers [10, 11]. This exchange process is slow compared to the hierachical assembly scheme described above. The homodimer vimentin molecule has a length of $44 \mathrm{~nm}$. In the assembled filament, the axial repeat length for vimentin is $43 \mathrm{~nm}$ [12].

In vitro, the assembly of vimentin is started by the addition of monovalent salts like potassium chloride or sodium chloride. Also, the addition of divalent ions leads to filament formation [14, 15. The assembly behaviour of IF proteins from different species have been investigated [16]. The temperature dependence shows the assembly is optimal at the body temperature of the respective species. When the optimal temperature is exceeded, filament formation is obstructed or inhibited. It was shown that the tail domain of vimetin is not necessary for the assembly [9]. After $10 \mathrm{~s}$ of assembly, ULFs are the dominant species at $0.1 \mathrm{~g} / \mathrm{l}$. Assembly of vimentin was shown even for concentrations as low as $0.005 \mathrm{~g} / 1$.

For studies of vimentin, two buffers systems are widely used. Assembly experiments are carried out mostly in a weak buffer containing phosphate and a varying salt concentration. This buffer has the advantage that the assembly is initiated only by changing the salt concentration in the buffer while the other parameters like $\mathrm{pH}$ value and buffer concentration are unchanged. For studying the vimentin structure, a buffer system containing 'TRIS' is widely used. In this buffer system, usually the buffer concentration, the salt concentration and the $\mathrm{pH}$ value are changed. Winheim et al. found that fila- 
ments in 'phosphate' buffer are thinner than in 'TRIS' buffer after an assembly time of 10 seconds at a concentration of $0.2 \mathrm{~g} / \mathrm{l}$ [17]. At this time point, the compaction step did not take place yet. Small changes to the amino acid sequence of vimentin can hinder the assembly into full filaments. The change of the tyrosine amino acid at position 117 into a leucine amino acid (mutant $Y 117 \mathrm{~L}$ ) causes the assembly to stop at the ULF stage [18.

The assembly of intermediate filaments has been studied with a variety of methods. For the assembly of tetramers to ULF, small angle X-ray scattering (SAXS) has been used [19, 20]. It was found that ULFs are formed on the time scale of one second. The assembly of ULFs to longer filaments can be tracked by transmission electron microscopy (TEM) 9] and atomic force microscopy (AFM) 21]. These techniques only allow for static measurements of the assembly states. Also dynamic light scattering (DLS) has been used to study the assembly of vimentin [22]. Full filaments have been studied with fluorescence microscopy 11, 17]. Portet et al. found with electron microscopy that the mean filament length for the assembly at $0.2 \mathrm{mg} / \mathrm{ml}$ after ten minutes is $480 \mathrm{~nm}$ or 11 ULF [23]. AFM was used to determine the persistence length [24]. The filaments were adsorbed to surfaces. This adsorption process alters the results of the persistence length measurement. The persistence length of vimentin filaments was measured with fluorescence microscopy techniques. A value of $2 \mu \mathrm{m}$ was found [25]. Here, the filaments were freely fluctuating in a constricted environment. The mechanics of assembled filaments was studied with AFM [24] and optical tweezers [26].

\subsection{Microfluidic techniques}

\subsubsection{Viscous flow}

The velocity field $\vec{v}$ of an incompressible, Newtonian fluid in a channel is governed by the Navier-Stokes equation:

$$
\rho\left(\frac{\partial \vec{v}}{\partial t}+(\vec{v} \nabla) \vec{v}\right)=-\nabla p+\eta \Delta \vec{v}+f_{\text {ext }}
$$

where $\rho$ is the fluid density, $\vec{v}$ the velocity field, $p$ the pressure, $\eta$ the dynamic viscosity and $f_{\text {ext }}$ an external force per volume. In the following, we introduce a renormalization 
to a characteristic velocity $U$, a characteristic time $\tau$ and a length scale $L$. The NavierStokes equation is then written in the dimensionless form without an external force and the primed quantities are the renormalized ones:

$$
\operatorname{Re}\left(\frac{\partial \vec{v}^{\prime}}{\partial t^{\prime}}+\left(\vec{v}^{\prime} \nabla^{\prime}\right) \vec{v}^{\prime}\right)=-\nabla^{\prime} p+\Delta^{\prime} \vec{v}
$$

Here, we also introduced the dimensionless Reynolds number:

$$
\operatorname{Re}:=\frac{\rho U L}{\eta}
$$

The Reynolds number is a measure for the ratio of the inertial and viscous forces in a hydrodynamic system. For Reynolds numbers above 1000 we get a turbulent flow, while below this number the flow is laminar. For microfluidic systems with water flowing in dimensions of $\mu \mathrm{m}$ and flow velocities of $\mathrm{mm} / \mathrm{s}$ we get $\mathrm{Re} \ll 1$. This value is way below the transitional Reynolds number which means that the flow in microflidic channels is mostly laminar.

For small Reynolds numbers, the term that stands for the inertial forces of Equation 2.2 vanishes and the flow is governed by the Stokes equation:

$$
\nabla p=\eta \Delta \vec{v}
$$

Without the inertial forces, only the pressure and the frictional term play a role. Since the inertia and time dependence are not contained in the equation anymore, no turbulence occurs. Thus we can assume laminar flow for microfluidic channels. In some microfluidic applications inertia plays a role [27].

\subsubsection{Diffusive mixing}

In the low Reynolds number regime, where no turbulences occur, mixing happens due to diffusion. The diffusion time $t$ of a molecule with diffusion coefficient $D$ along a distance 
$x$ in three dimensions is given by:

$$
\left\langle x^{2}\right\rangle=6 D \tau
$$

One measure for the diffusive mixing in a micro-channel is the Péclet number [28]. When two solutions are brought into contact at a channel junction, the time $\tau$ until complete diffusive mixing is given by:

$$
\tau \sim \frac{w^{2}}{D}
$$

with $w$ being the channel width and $D$ being the diffusion coefficient. During this time $\tau$ the solution moves down the channel with the flow velocity $v$.

$$
s=v \tau \sim \frac{v w^{2}}{D}
$$

By dividing by the channel width $w$ we arrive at:

$$
\frac{s}{w} \sim \frac{v w}{D}=: \mathrm{Pe}
$$

where we introduce the dimensionless Péclet number. It measures the distance along the channel after which a solution is fully mixed by diffusion in terms of the channel width.

For a microfluidic device with a typical length scale of $w=100 \mu \mathrm{m}$ and a flow velocity of $v=1 \mathrm{~mm} / \mathrm{s}$ and $D=10 \mathrm{\mu m}^{2} / \mathrm{s}$, the Péclet number is $10^{4}$. For mixing of salt molecules ( $D=1000 \mathrm{\mu m}^{2} / \mathrm{s}$ ), the Péclet number is 100 . This means that the mixing of the smaller particles happens faster than for the larger molecules in the same channel at the same flow velocity.

\subsubsection{Microfluidic mixers}

Microfluidic techniques are an emerging tool for studying the dynamics of proteins. One advantage is the fact that only small amounts of protein are used. The reactants come into contact after short dead times. Microfluidic mixers for studying protein kinetics use a continuous flow scheme. The protein stream comes into contact with a buffer solution that contains trigger molecules. Microfluidic mixers have been used for studying protein 


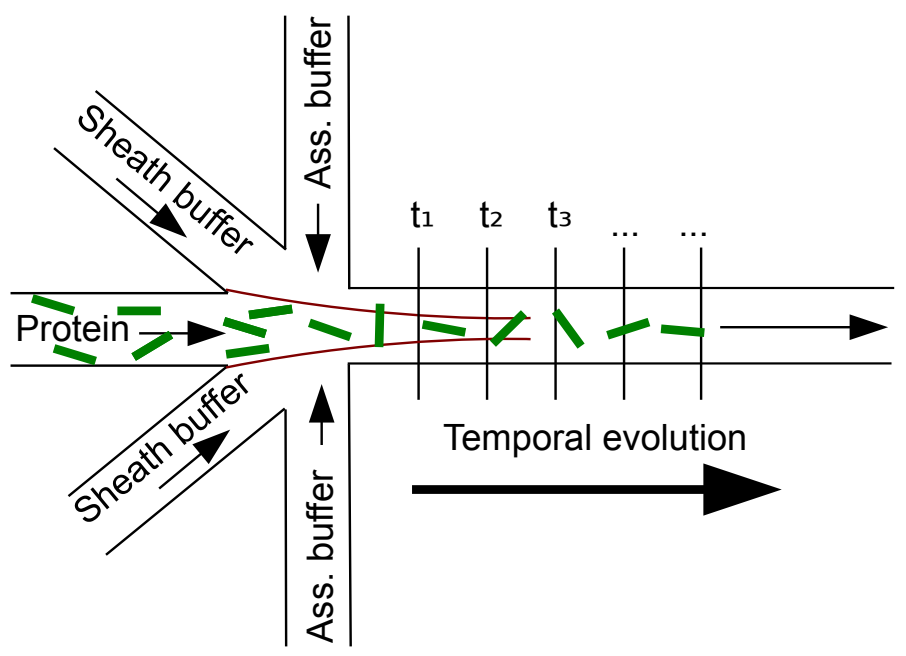

Figure 2.3: Sketch of a five-inlet mixing device. The diagonal sheath flows focus the central jet before it comes into contact with the side flows that contain salt. The distribution of reaction times lowered by this approach.

folding. For example, the protein stream contains a chaotropic agent that keeps the protein in an unfolded state [29]. The folding is initiated by unmixing the chaotropic agent from the protein jet. It is also possible to study the reaction of a protein to the addition of a trigger molecule, for example the binding of $\mathrm{Ca}^{2+}$ to calmodulin [30]. By adjusting the flow rates, the inner stream can be narrowed down to a small width. This effect is called hydrodynamic focusing [31, 32]. Trigger molecules from the outer stream only need to diffuse short distances to fully spread into the inner stream. This effect is increased by narrowing down the width of the inlets [33, 34]. To achieve fast mixing and maintaining a slow enough flow velocity in the measuring outlet, one can employ a device where the channel is very narrow directly after the mixing region and then widens up when the fluid is completely mixed (see Figure 2.4). The narrow channel ensures a short mixing time. This geometry ensures sufficient residence times of the molecules in the detection volume to measure the fluorescent signal, while the mixing happens on a fast time scale.

The basic layout for a microfluidic mixer is comprised of three inlets. By adding additional diagonal inlets between the center and the side inlets the central stream is hydrodynamically focused before the diffusive mixing starts [35]. A sketch of the fiveinlet mixer is depicted in Figure 2.3. To prevent protein adsorption in a microfluidic mixer there is the possibility of decreasing the height of the protein inlet [20, $36-38]$. 


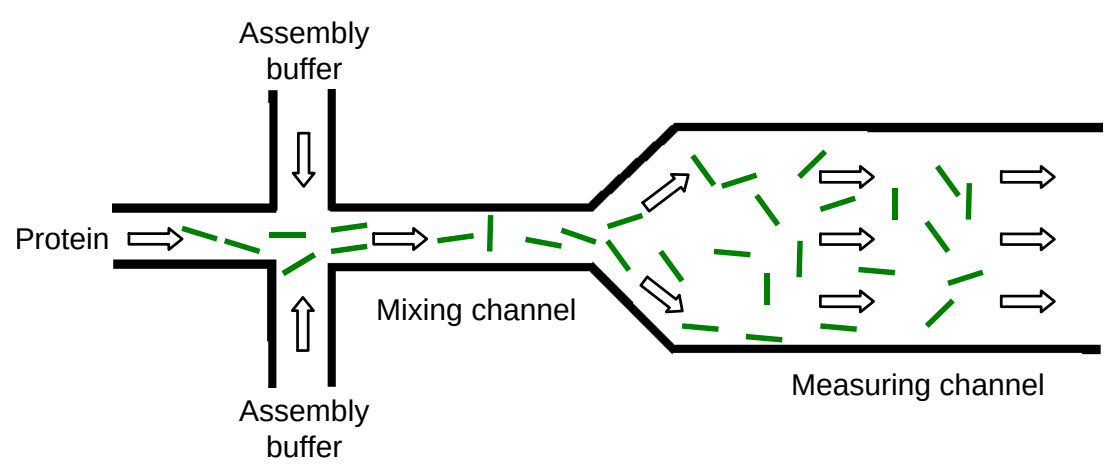

Figure 2.4: Sketch of a three-inlet device with a bottleneck structure. The solutions are mixed in the narrow channel after the mixing region via diffusion. The following wider channel leads to a lower flow velocity. Measurements of the sample take place in this region.

The outer streams surround the inner stream in this configuration also from the top and the bottom and the contact of the inner stream to the channel walls is minimized.

Chaotic mixing is used in the so-called herringbone mixer [39 41]. Diagonal grooves on the top and bottom side of the channel cause the fluid stream to rotate. This leads to a continuous folding of fluid layers. The distance that reactants need to diffuse in order to spread evenly is minimized. This leads to lower mixing times. For this kind of mixer a complex design and fabrication process is necessary.

Diffusion of cylindrical rod The diffusion coefficient of a stiff cylindrical rod is described by the following equation 42,44$]$ :

$$
D=\frac{k_{\mathrm{B}} T}{3 \pi \eta_{0} L} \cdot\left(\ln \left(\frac{L}{d}\right)+0.3+0.6 \frac{d}{L}-0.1 \frac{d^{2}}{L^{2}}\right)
$$

where $L$ and $d$ are the length and the diameter of the cylinder, respectively, $\eta_{0}$ is the viscosity of water, $k_{\mathrm{B}} T$ is the Boltzmann factor. An estimation for the dependency of the diffusion coefficient of a vimentin filament on the filament length is shown in Figure 2.5. The curve does not take into account the the varying diameter of short filaments. The decrease of the diffusion coefficient for filaments between $2 \mu \mathrm{m}$ and $10 \mu \mathrm{m}$ is only small. 


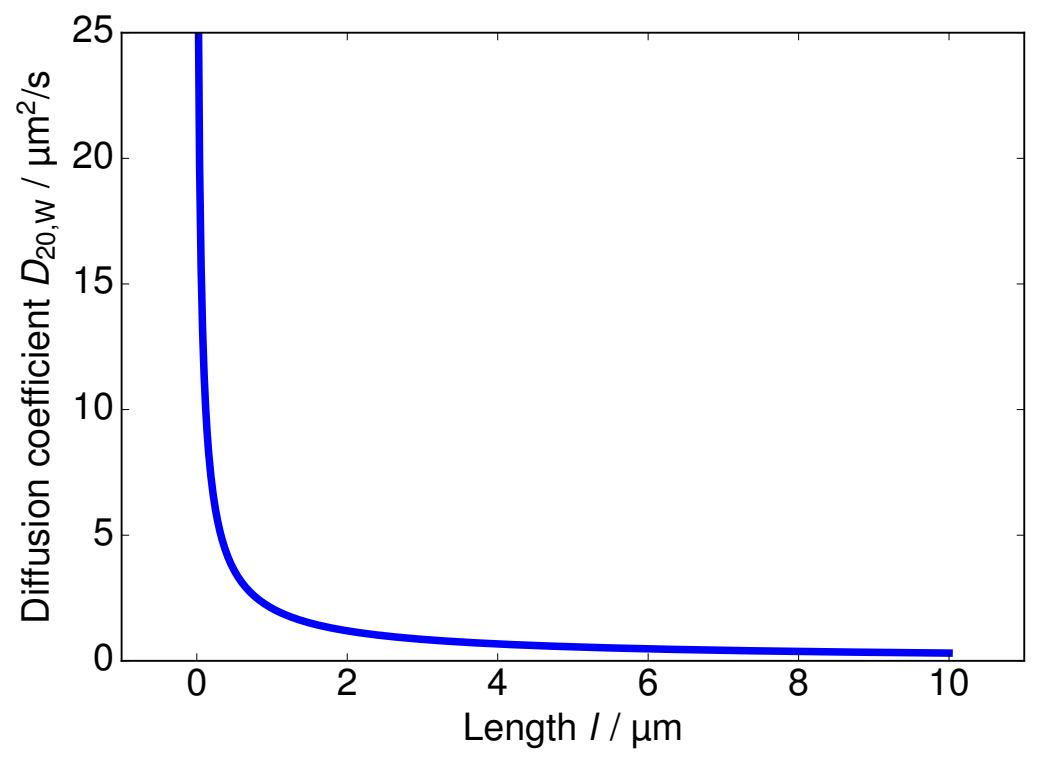

Figure 2.5: Diffusion coefficients of vimentin filaments Estimation of the diffusion coefficient for vimentin filaments. The curve does not take into account the varying diameter of short filaments.

\subsection{Fluorescence correlation spectroscopy}

Fluorescence correlation spectroscopy (FCS) is a versatile technique to study the dynamics of fluorescent molecules. The technique is based on measuring the fluctuations of a fluorescent signal in a small detection volume 45 48. Fluorescent molecules are excited with laser light (see Figure 2.6). The emitted light is detected.

For further analysis, the correlation function of the detected signal is calculated. The shape of the resulting correlation curve is governed by the molecular system and the geometry of the detection volume. The geometry of the detection volume is assumed to have a Gaussian shape. In order to detect the fluctuations of molecules diffusing in and out of the detection volume, the number of particles needs to be low. Otherwise the fluctuations are to small in comparison to the total signal.

\subsubsection{Fluorescence}

Fluorescence is the emission of a photon with a longer wavelength after absorption of an incident photon. During the excitation electrons are elevated to a higher energy state. 


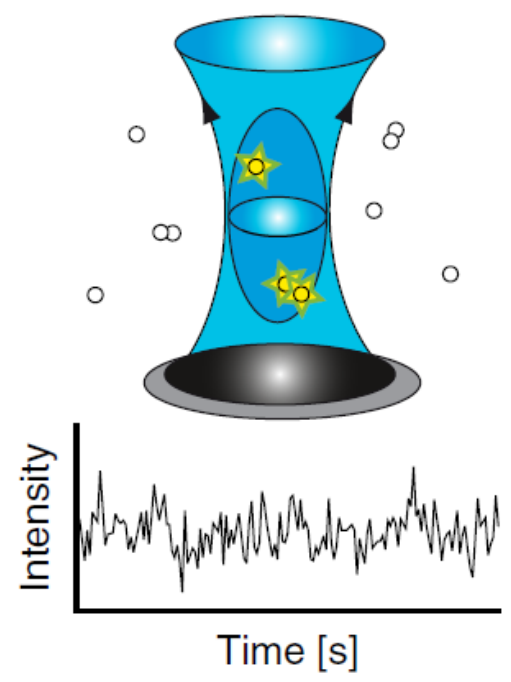

Figure 2.6: Signal detection in FCS. Fluorescent molecules that diffuse in and out of a confocal volume cause intensity fluctuations. These fluctuations are analysed in FCS. Image with permission from 49$]$.

Higher vibrational states relax to the lowest vibrational state without the emission of light. A photon is emitted when the electron relaxes to the ground state. The lifetime of these states is of the order of nanoseconds. The difference between the excitation wavelength and the emission wavelength is called Stokes shift.

$$
\Delta \lambda_{\text {Stokes }}=\lambda_{\text {exc }}-\lambda_{\text {emi }}
$$

The energy states in the molecule are represented in a Jablonski diagram (see Figure 2.7). The triplet state is reached by intersystem crossing. The triplet state dynamics play a role in the FCS measurements (see below).

The confocal volume is shaped by focusing a Gaussian laser beam. The radius $r_{0}$ of the focused beam is approximated by [50]:

$$
r_{0} \approx \frac{f \lambda_{\mathrm{exc}}}{n \pi R}
$$

where $f$ is the focal length of the objective, $\lambda_{\text {exc }}$ is the wavelength of the excitation light, $n$ is the refraction index of the medium, and $R$ is the radius of the incident beam. 


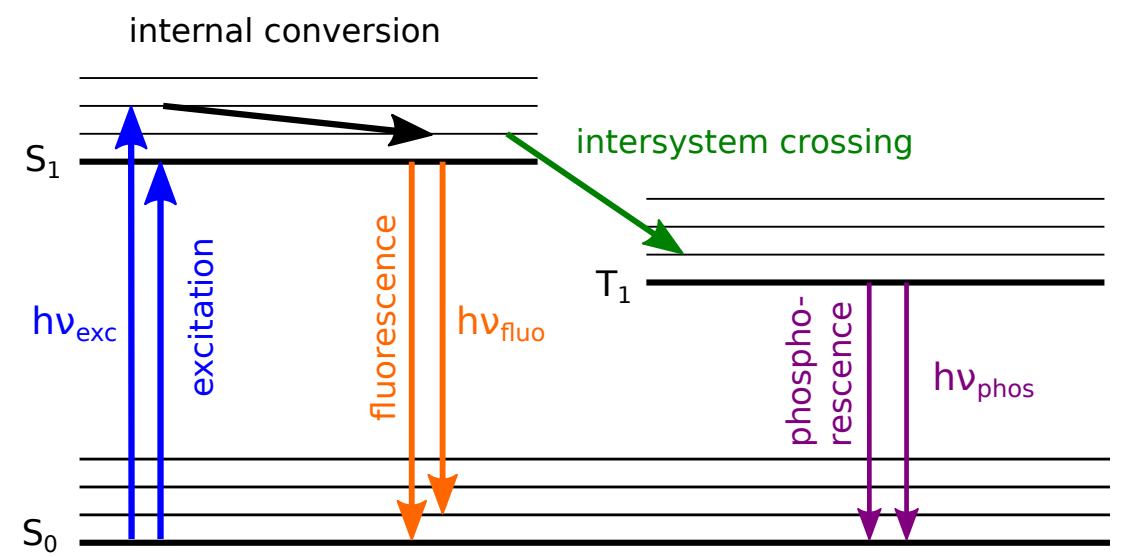

Figure 2.7: Jablonski diagram The energy states of a fluorescent molecule are depicted in the Jablonski diagram. The transition between the singlet states $S_{0}$ and $S_{1}$ represent the emission of fluorescence. The triplet states are reached from the singlet states by intersystem-crossing.

\subsubsection{Theory of fluorescence correlation spectroscopy}

The fluorescent signal depends on the time dependent distribution of fluorophores in the detection volume $c(\vec{r}, t)$ and the normalized instrumental detection efficiency $W(\vec{r})[51$. The total signal $F(t)$ is the integral over all photons coming from the detection volume.

$$
F(t)=q \int W(\vec{r}) c(\vec{r}, t) \mathrm{d} V
$$

The factor $q$ stands for the photo-physical properties of fluorescence emission like absorption efficiency and quantum yield.

In the following equations the angular brackets denote the time average. By applying the ergodic theorem which states that time average and the ensemble average are equivalent we get:

$$
\langle F(t)\rangle=\lim _{t \rightarrow \infty} \int F(t) \mathrm{d} t=q \int W(\vec{r}) c \mathrm{~d} V=q n
$$

The function for the detection volume $W(\vec{r})$ can be approximated by a Gaussian in three dimensions. The function decays to $1 / \mathrm{e}^{2}$ at a distance of $w_{\mathrm{xy}}$ from the center in 


\section{State of the art and theoretical background}

the lateral direction and at a distance of $w_{\mathrm{z}}$ in the axial direction.

$$
W(\vec{r})=\exp \left(-2 \frac{x^{2}+y^{2}}{w_{\mathrm{xy}}^{2}}\right) \exp \left(-2 \frac{z^{2}}{w_{\mathrm{z}}^{2}}\right)
$$

The normalized auto-correlation function is defined as:

$$
G(\tau)=\frac{\langle F(t) \cdot F(t+\tau)\rangle}{\langle F\rangle^{2}}-1=\frac{\langle\delta F(t) \cdot \delta F(t+\tau)\rangle}{\langle F\rangle^{2}}
$$

The signal is compared to itself after the lag time $\tau$. For a completely uncorrelated signal, the ACF yields a value of zero. When the signal contains a correlation, the value of the ACF is greater than zero.

The correlation function can be written with the diffusion propagator:

$$
\langle F(t) \cdot F(t+\tau)\rangle=\frac{q^{2}}{V} \iint W(\vec{r}) P_{\mathrm{d}}\left(\vec{r}^{\prime} \mid \vec{r}, \tau\right) W\left(\vec{r}^{\prime}\right) \mathrm{d} V \mathrm{~d} V^{\prime}
$$

The diffusion propagator is given by:

$$
P_{\mathrm{d}}\left(\vec{r}^{\prime} \mid \vec{r}, \tau\right)=\frac{1}{\left(4 \pi D_{\mathrm{t}} \tau\right)^{3 / 2}} \exp \left(-\frac{\left|\vec{r}^{\prime}-\vec{r}\right|}{4 D_{\mathrm{t}} \tau}\right)
$$

By introducing Equation (2.17) into Equation 2.16 we can write $G(\tau)$ as:

$$
G(\tau)=\frac{1}{N}\left(1+\frac{4 D_{\mathrm{t}} \tau}{w_{\mathrm{xy}}^{2}}\right)^{-1}\left(1+\frac{4 D_{\mathrm{t}} \tau}{w_{\mathrm{z}}^{2}}\right)^{-1 / 2}
$$

and with

$$
\tau_{\text {diff }}=\frac{w_{\mathrm{xy}}^{2}}{4 D_{\mathrm{t}}}, \gamma=\frac{w_{\mathrm{xy}}}{w_{\mathrm{z}}}
$$

we get:

$$
G(\tau)=\frac{1}{N}\left(1+\frac{\tau}{\tau_{\text {diff }}}\right)^{-1}\left(1+\frac{\tau}{\gamma^{2} \tau_{\text {diff }}}\right)^{-1 / 2}
$$

In the experiment, the values for $w_{\mathrm{xy}}$ and $w_{\mathrm{z}}$ have to be obtained by measuring the diffusion of a molecule with a known diffusion coefficient. Absolute values for the diffu- 
sion coefficient are obtained by comparing the value to a standard. With the knowledge of these values it is possible to determine absolute diffusion coefficients. The accuracy of the measurement of the calibration standard determines the absolute accuracy of the measurement. Techniques for determining absolute diffusion coefficients include dualfocus FCCS [52] and scanning FCS [53].

For two diffusing components with differing diffusion coefficients we use the following equation where $\rho$ describes the ratio between the components assuming they have the same brightness:

$$
G_{3 \mathrm{D}, \mathrm{comp} 2}(\tau)=\frac{1}{N}\left(\rho_{1} \cdot g_{1}(\tau)+\left(1-\rho_{1}\right) \cdot g_{2}(\tau)\right)
$$

with

$$
g_{i}(\tau)=\frac{1}{1+\tau / \tau_{\mathrm{diff}, i}} \sqrt{\frac{1}{1+\tau /\left(\gamma^{2} \tau_{\mathrm{diff}, i}\right)}}
$$

FCS in flow With FCS we can also measure molecules that are driven by an active flow. For uniform translation with a constant velocity $v$ and without diffusion $(D=0)$ the following equation holds [48]:

$$
G(\tau)=\frac{1}{N} \exp \left(-\left(\frac{v \tau}{w_{\mathrm{xy}}}\right)^{2}\right)
$$

For a uniform flow velocity and free diffusing molecules we use the following equation:

$$
G_{3 \mathrm{D}, \text { flow }}(\tau)=G_{3 \mathrm{D}}(\tau) \cdot \exp \left(-\left(\frac{\tau}{\tau_{\text {flow }}}\right)^{2} \cdot\left(1+\frac{\tau}{\tau_{\text {diff }}}\right)^{-1}\right)
$$

with

$$
\tau_{\text {flow }}=\frac{2 w_{\mathrm{xy}}}{v}
$$

The technique of FCS has been used to determine the flow profiles in microfluidic channels [54, 55]. With one focus FCS and a known coefficient of the flowing sample, the flow profile can be determined accurately [54]. In these studies the diffusion coefficient 
of the measured molecules was known and fixed in the fit. This improves the accuracy for determining the flow velocity in the fit.

\subsubsection{Further aspects of FCS}

Triplet dynamics Fluorescent molecules absorb light and emit light at a longer wavelength. During this process, electrons are excited from the ground state into higher states. They relax to the ground state by emitting photons. These processes have lifetimes on the order of nanoseconds. Triplet states are reached by intersystem crossing. The lifetime of the triplet state is on the order of microseconds. The triplet dynamics cause a correlation which shows up in the FCS measurements [56]. The triplet part of an ACF is described with the following term:

$$
T(\tau)=\frac{1-\theta_{\mathrm{T}}+\theta_{\mathrm{T}} \exp \left(-\tau / \tau_{\mathrm{T}}\right)}{1-\theta_{\mathrm{T}}}
$$

where $\theta_{\mathrm{T}}$ is equilibrium population and $\tau_{\mathrm{T}}$ is the lifetime of the triplet state.

Afterpulsing An aspect that needs to be considered during FCS measurements is afterpulsing. Afterpulsing is caused by feedback in the photon detector. A real detected photon can cause an afterpulse after a short time span. This effect shows up as a strong correlation in the ACF at short lag times. There are measures to treat this effect theoretically by introducing an additional component in the fit [57 Cross-correlation does not show afterpulsing because the afterpulsing effects from two detectors are statistically independent. When fitting the data, it is possible to only include the lag times that do not contain the afterpulsing effect.

Further developments of FCS The technique of FCS was developed further. In fluorescence cross-correlation (FCCS) the signal of two separate detectors is cross-correlated. With two-color FCCS it is possible to detect binding of differently labeled molecules [58]. When two differently labeled molecules show a cross-correlation, the two molecules are bound to each other. Two different molecules can be excited by separate laser sources or by the same laser source (single wavelength FCS, SW-FCCS) [59]. In dual focus FCCS two parallel focus spots are used [60,61]. The distance between the focus spots is fixed and can be calculated. This provides a possibility to calibrate the system without the 
measurement of a calibration standard.

In Scanning FCS (SFCS) the laser beam is scanned periodically across the sample [62]. In circular-scanning FCS, the scanning radius can be determined precisely [53]. This makes it possible to measure absolute diffusion coefficients. 
2 State of the art and theoretical background 


\section{Materials and methods}

\subsection{Preparation of vimentin}

\subsubsection{Fluorescent labeling of vimentin}

Human vimentin is expressed in Escherichia coli (E. coli) bacteria. The vimentin plasmid was provided by Harald Herrmann, DKFZ Heidelberg, Germany. The protein is produced following the protocol of Herrmann et al. [63].

We label vimentin with fluorescent dye molecules via a maleimide reaction. The target of the maleimide is the amino acid cysteine. Vimentin contains one cysteine at position 328. Since the binding of the dye in the rod domain of the protein interferes with the assembly process, we use a vimentin mutant that has the cysteine replaced by an alanine and a cysteine is added to the C-terminus. This mutant is referred to as vimentin-CYS hereafter.

The labeling protocol is based on Winheim et al. [17]. Vimentin-CYS stored in $8 \mathrm{M}$ urea is dialyzed into labeling buffer $(5 \mathrm{M}$ urea, $50 \mathrm{mM}$ phosphate buffer, $\mathrm{pH} 7)$ for two hours at room temperature. The protein concentration is diluted to $1 \mathrm{~g} / \mathrm{l}$ with labeling buffer. The maleimide dye is solubilized in water-free DMSO to a concentration of $10 \mathrm{mM}$. The dye is added to the protein in $5 \mu \mathrm{l}$ portions until a molar excess of 1:20 is reached. After each addition of dye the solution is briefly vortexed. The solution is incubated for two hours. Afterwards, free dye is captured by the addition of $1 \mathrm{M}$ cysteine in water to a concentration of $100 \mathrm{mM}$ and incubated for two hours. The free dye is separated from the labeled protein via size exclusion chromatography. A $30 \mathrm{~cm}$ column is filled with column resin (BioGel P-30, Bio-Rad) equilibrated in labeling buffer and the protein solution is added. The labeled protein is eluated before the free dye and collected in fractions. The protein and dye concentration are determined with a NanoDrop ND-1000 spectrophotometer (NanoDrop Technologies Inc., Wilmington, USA). Peak fractions are pooled. The labeled protein is dialyzed against storage buffer 
Table 3.1: Fluorescent dyes used in this thesis project. The molecular weight is given for the dyes with a maleimide group.

\begin{tabular}{cccccc}
\hline Dye & $\begin{array}{c}\lambda_{\text {exc }} / \\
\mathrm{nm}\end{array}$ & $\begin{array}{c}\lambda_{\text {emi }} / \\
\mathrm{nm}\end{array}$ & $\begin{array}{c}\text { Quantum } \\
\text { yield }\end{array}$ & $\begin{array}{c}\text { Ext. coeff. / } \\
\mathrm{cm}^{-1} \mathrm{M}^{-1}\end{array}$ & $\begin{array}{c}\text { Mol. weight / } \\
\mathrm{gmol}^{-1}\end{array}$ \\
\hline \hline Alexa Fluor 532 & 528 & 552 & 0.61 & 78,000 & 812.88 \\
ATTO 532 & 532 & 553 & 0.90 & 115,000 & 1063 \\
Dyomics DY-521XL & 523 & 668 & 0.02 & 50,000 & 752.80 \\
\hline
\end{tabular}

Table 3.2: Labeling ratios and expected number of labeled monomers in a tetramer

\begin{tabular}{cc}
\hline Ratio & Labeled monomers \\
\hline \hline 0.01 & 1.02 \\
0.025 & 1.04 \\
0.05 & 1.08 \\
0.1 & 1.16 \\
\hline
\end{tabular}

(8 $\mathrm{M}$ urea, $2 \mathrm{mM}$ phosphate buffer, $\mathrm{pH} 7.5$ ), aliquoted and stored at $-80^{\circ} \mathrm{C}$. The dyes used in this thesis are listed in Table 3.1.

\subsubsection{Label distribution}

The distribution of labeled monomers is given by the binomial distribution. The probability to have $k$ labeled molecules in a polymer consisting of $n$ monomers in total is:

$$
p_{n}^{k}=\left(\begin{array}{l}
n \\
k
\end{array}\right) r^{k}(1-r)^{n-k}
$$

The labeling ratios between labeled and unlabeled vimentin and corresponding expectation values of labeled monomers in a tetramer are shown in Table 3.2 .

\subsubsection{Dialysis and assembly}

Labeled and unlabeled vimentin are mixed in a molar ratios between 0.01 and 0.1 (labeled:unlabeled). The protein is dialyzed step-wise from $8 \mathrm{M}$ urea to $0 \mathrm{M}$ urea, $2 \mathrm{mM}$ phosphate buffer, pH 7.5 via the following steps: $6 \mathrm{M}, 4 \mathrm{M}, 2 \mathrm{M}, 1 \mathrm{M}$. Every step is carried out for 30 minutes at room temperature. Afterwards the protein is dialyzed against 
$2 \mathrm{mM}$ phosphate buffer, $\mathrm{pH} 7.5$ overnight at $10^{\circ} \mathrm{C}$ and for 30 minutes in degassed buffer the next day.

For bulk assembly, the vimentin solution is diluted with phosphate buffer to concentrations between $20 \mathrm{mg} / \mathrm{l}$ and $200 \mathrm{mg} / \mathrm{l}$ and mixed $1: 1$ with $200 \mathrm{mM} \mathrm{KCl}, 2 \mathrm{mM}$ phosphate buffer, pH 7.5. The mixed solution is incubated at $37^{\circ} \mathrm{C}$. For filament imaging, the vimentin is assembled for 24 hours.

\subsection{Numerical simulations}

Finite element simulations (FEM) are performed with COMSOL Multiphysics 5.0 (COMSOL, Göttingen). The software subdivides the model and solves the partial equations of the physical problem individually for every element. All channels of the mixing device have a width of $200 \mu \mathrm{m}$. The height of the central inlet channel (protein channel) is $50 \mu \mathrm{m}$, while the height of the other inlet channels and the outlet channel is $100 \mu \mathrm{m}$ (see Figure 3.1). The channel walls are represented by boundaries with a no-slip condition. The model is meshed with a minimum mesh size of $1.5 \mu \mathrm{m}$ and a maximum mesh size of $7.8 \mu \mathrm{m}$. Since the microfluidic device is symmetric in two axes, only a quarter of the device is simulated. The flow material is water with the addition of solutes. The diffusion of vimentin is modeled with a diffusion coefficient of $D_{\mathrm{Vim}}=24 \mu \mathrm{m}^{2} / \mathrm{s}$. For the potassium chloride ions, a diffusion coefficient of $D_{\mathrm{KCl}}=1850 \mu \mathrm{m}^{2} / \mathrm{s}$ is used [64].

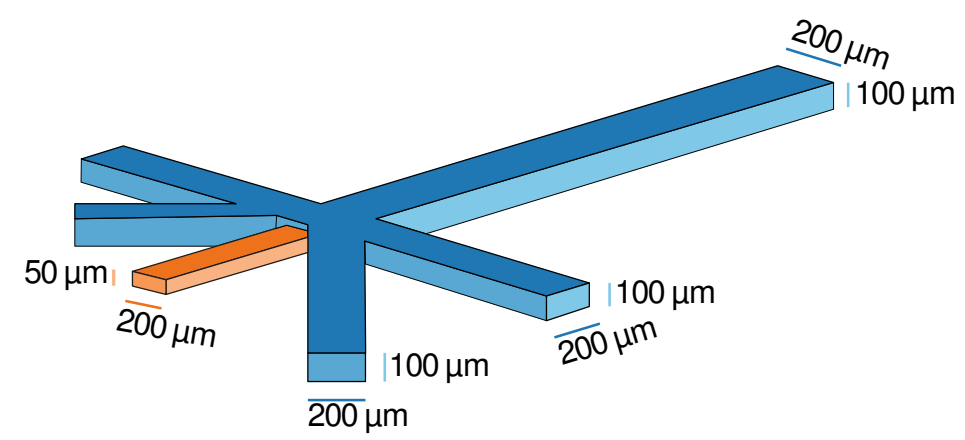

Figure 3.1: Sketch of the five-inlet device with constraining step. 
Table 3.3: Spin-coating procedure for resist layers of different heights

\begin{tabular}{ccccc}
\hline $\begin{array}{c}\text { Height / } \\
\mu \mathrm{m}\end{array}$ & Resist & $\begin{array}{c}\text { Duration / } \\
\mathrm{s}\end{array}$ & $\begin{array}{c}\text { Speed / } \\
\mathrm{rpm}\end{array}$ & $\begin{array}{c}\text { Ramp / } \\
\mathrm{rpm} / \mathrm{s}\end{array}$ \\
\hline \hline 25 & SU8-3025 & 5 & 500 & 100 \\
& & 30 & 3500 & 300 \\
\hline 75 & SU8-3050 & 5 & 500 & 100 \\
& & 30 & 1700 & 300 \\
\hline 100 & SU8-3050 & 5 & 500 & 100 \\
& & 30 & 1300 & 300 \\
\hline \hline
\end{tabular}

\subsection{Microfluidic devices}

\subsubsection{Photo lithography}

All lithography steps are carried out in the class 1000 clean room of the physics faculty at the University of Göttingen. 2-inch silicon wafers are washed with iso-propanol and blown dry with nitrogen. To evaporate remaining iso-propanol, the wafers are heated to $195^{\circ} \mathrm{C}$ for $20 \mathrm{~min}$. The photo resist (SU8-3025 or SU8-3050, MicroChem.) is spincoated with the sequence listed in Table 3.3 and softbaked at $95^{\circ} \mathrm{C}$ for $45 \mathrm{~min}$.

To produce the structures, the wafers are exposed with UV light $(365 \mathrm{~nm})$ through a photomask on a mask aligner (MJB4, Suess MicroTec, Garching, Germany, Vacuum mode: Vac, Prevac: $10 \mathrm{~s}$, Mainvac: $20 \mathrm{~s}$, Intensity: $22 \mathrm{~mW} / \mathrm{cm}^{2}$, Exposure time: $10 \mathrm{~s}$ for $25 \mu \mathrm{m}, 17 \mathrm{~s}$ of $75 \mu \mathrm{m}$, and $20 \mathrm{~s}$ for $100 \mu \mathrm{m}$. The wafers are then baked to further fixate the formed structure $\left(1 \mathrm{~min}\right.$ at $65^{\circ} \mathrm{C}, 10 \mathrm{~min}$ at $\left.95^{\circ} \mathrm{C}\right)$. Two-layer wafers are spincoated, soft baked, and exposed twice with the procedures corresponding to the desired layer

(a) Si wafer

(c) Exposure

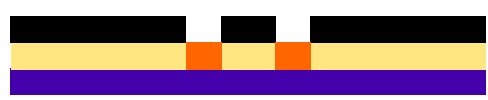

(b) Photo resist

(d) Development

Figure 3.2: Photo lithography steps for the fabrication of master wafer (a) Silicon wafer (2 inch). (b) Spincoating with photo resist. (c) Exposure through photo mask. (d) Development of the photo resist. 
height. The second photomask is aligned to the previous structure with the microscope of the mask aligner and exposed. Afterwards, the wafers are developed for 20 minutes with SU8 developer and hardbaked for 5 minutes at $150{ }^{\circ} \mathrm{C}$. The structured wafers are vaporcoated with (heptafluoro- propyl)-trimethylsilane (Aldrich, Steinheim, Germany) to facilitate the removal of the cured PDMS from the wafer. For this, $20 \mu l$ of the fluorosilane are placed in a small dish. The fluorosilane and the wafers are placed in a desiccator. The desiccator is evacuated for two hours or overnight.

\subsubsection{Soft lithography}

\section{PDMS molding}

We use polydimethylsiloxane (PDMS, Sylgard 184 elastomere kit, Dow Corning, Midland, MI, USA) to form the microfluidic channels [65]. To produce PDMS molds of the structured silicon wafers, a mixture of $10: 1$ of PDMS and crosslinker is poured onto the wafer. The PDMS is degassed in a desiccator to remove air bubbles. The PDMS is then cured for at least one hour at $65{ }^{\circ} \mathrm{C}$ in an oven. The cured PDMS is cut out from the wafer and cleaned with isopropanol (see Figure 3.6). The isopropanol is blown off with nitrogen and remaining isopropanol is removed by placing the PDMS onto a hotplate at $95^{\circ} \mathrm{C}$. Holes for connecting the tubings are punched into the PDMS channels. To bond the PDMS slab to a cover glass slide, both are exposed to a plasma in a plasma cleaner for 10 seconds (PDC-32G, Harrick Plasma, Ithaca, New York, USA). The PDMS slab is pressed onto the glass slide while sitting on the $95^{\circ} \mathrm{C}$ hotplate to form a covalent bond between the two pieces. Two cross-sections of channels in PDMS of an inlet channel and a two-step outlet channel are shown in Figure 3.3.

\section{Step structure for constraining the inflow}

To constrict the inflow of the protein jet, we produce a step structure on a glass slide using micromolding in capillaries (MIMIC) [66]. The process is depicted in Figure 3.4 . We use a PDMS slab with a channel that is cut open on one side and place a droplet of the UV-curable glue NOA83H (Norland Products, Inc., Cranbury, NJ, USA) at the open end. The channel is $200 \mu \mathrm{m}$ wide and $25 \mu \mathrm{m}$ high. The glue is then dragged into the channel by capillary forces. Because of the viscosity of the glue, this step takes several hours. The complete filling by capillary forces is faster for the gue NOA83H than for the 


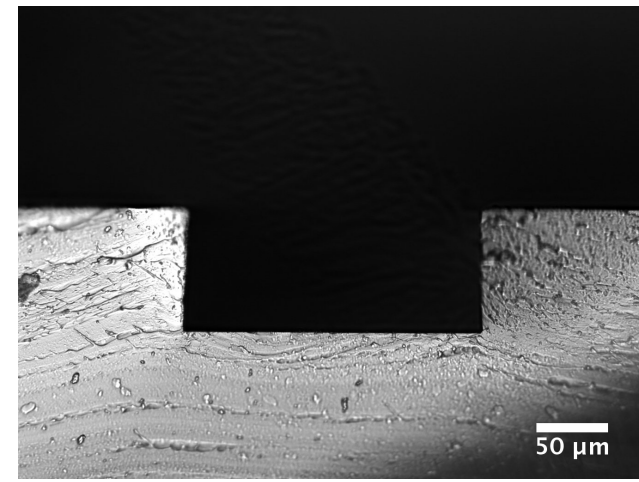

(a)

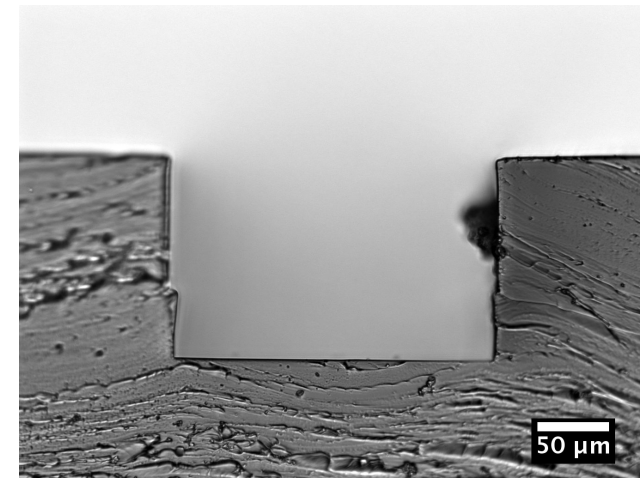

(b)

Figure 3.3: Cross-section of PDMS channels. The PDMS channel is cut perpendicular to the channel direction and imaged on a brightfield microscope. (a) Inlet channel width decreased height. (b) Outlet channel produced with a two-step procedure. The sidewalls of the lower part of the channel show the transition between the two layers in the SU8 master.

NOA81. The air in the channel moves into the PDMS while the channel is filled due to the gas permeability of the PDMS. When the channel is filled completely, the remaining glue at the inlet of the channel is removed with paper tissue and isopropanol. The glue is cured by exposing it to UV light of $365 \mathrm{~nm}$ overnight. Afterwards the PDMS slab is removed. For further hardening, the glue is again exposed to UV light for one hour (see Figure 3.5). After wiping off the remaining glue at the inlet, a line of glue remains at intersection of PDMS and glass. When the structured PDMS slab is applied, this glue bump can hold up the slab and lead to a connection between the inlet channels. Connections between the inlet channel may prevent the proper function of the flow device.

The catalyst in the NOA glue diffuses into the PDMS over time [67]. After some time the glue does not harden fully anymore, even after excessive exposure. Especially in the tip region of the channel the glue stays soft. To ensure complete hardening, exposure needs to start right after complete filling of the channel. The length of the channel should be as short as necessary to minimize filling time. 
(1)

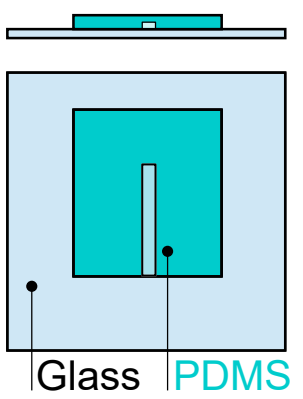

(4)

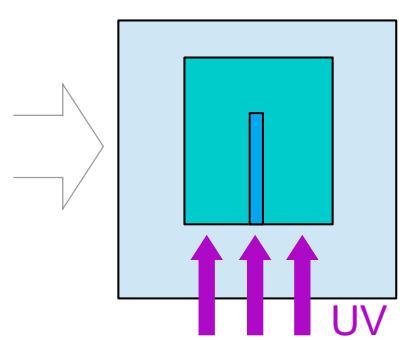

(2)

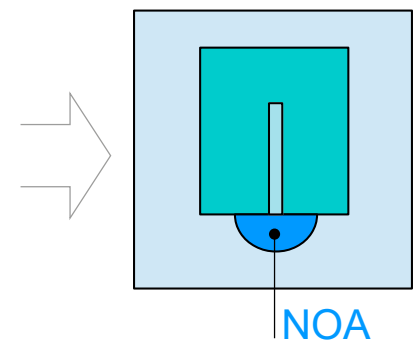

(5)

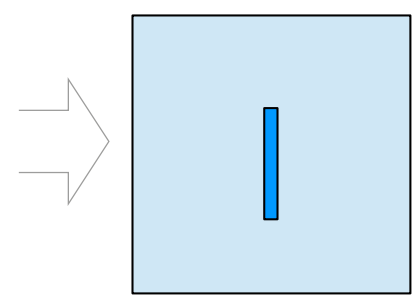

(3)

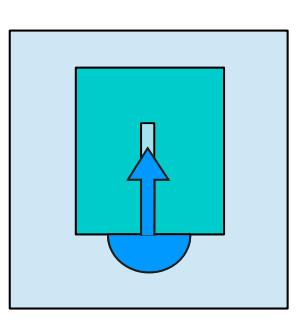

(6)

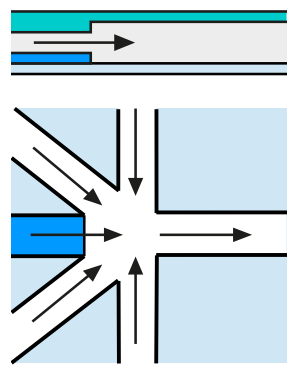

Figure 3.4: Molding of step structure. (1) A piece of PDMS with a channel structure is placed on glass. (2) A drop of UV-curable glue is placed at the inlet of the channel. (3) The glue is dragged into the channel by capillary forces. After complete filling of the channel, the remaining glue drop is removed. (4) The glue is hardened by exposing it to UV light. (5) The PDMS slab is removed. (6) Scheme of full microfluidic device.

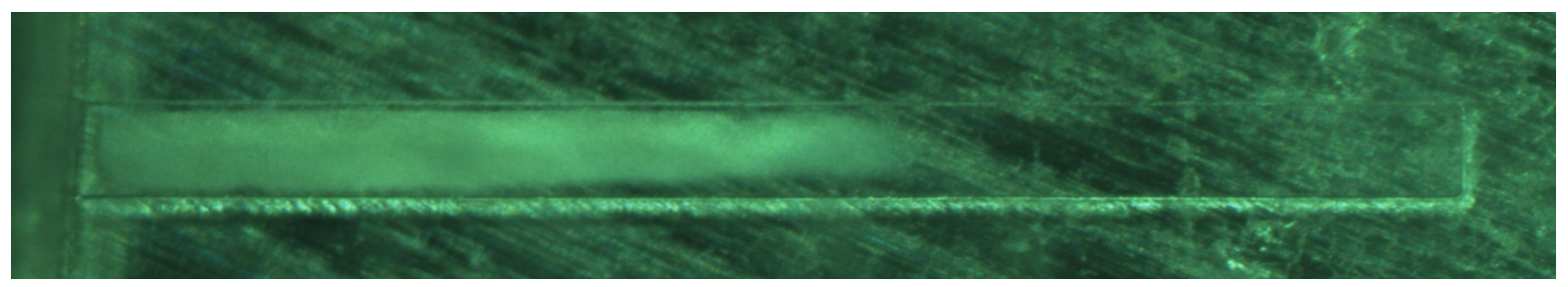

Figure 3.5: Image of a molded step structure. The edge of the PDMS slab is visible to the left side of the structure. 


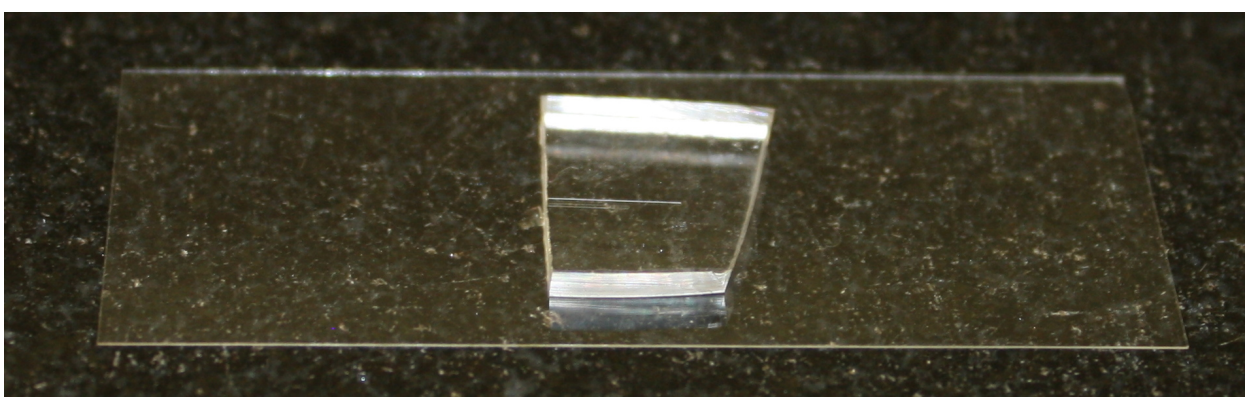

(a)

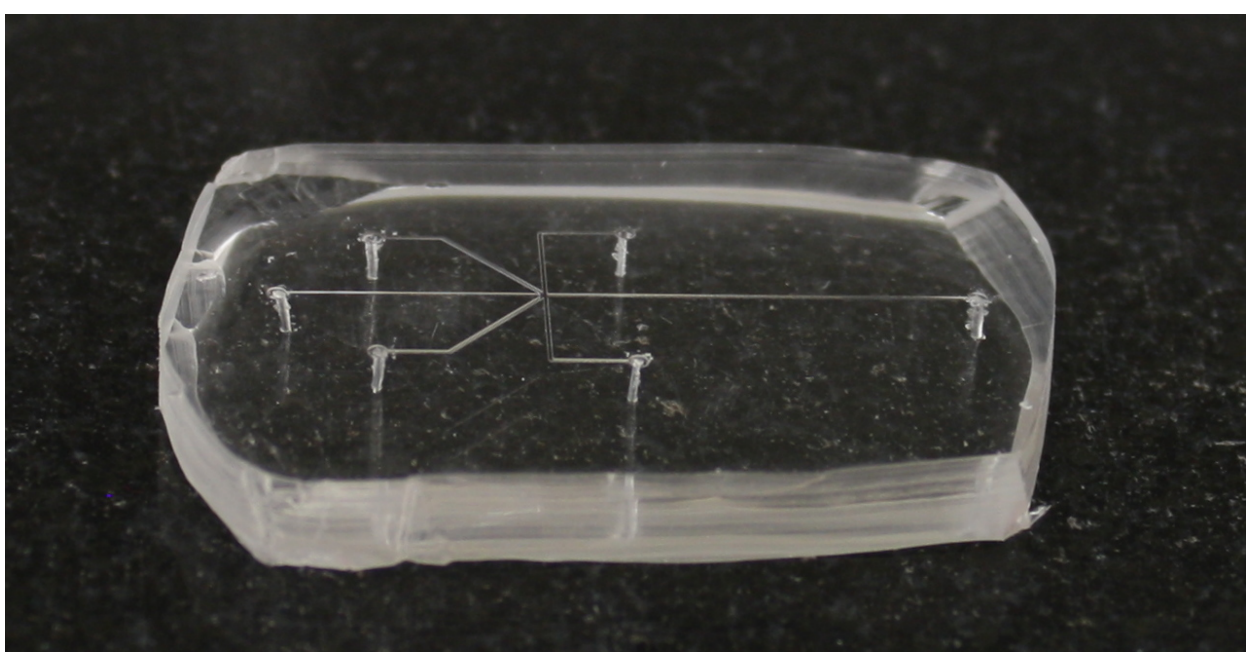

(b)

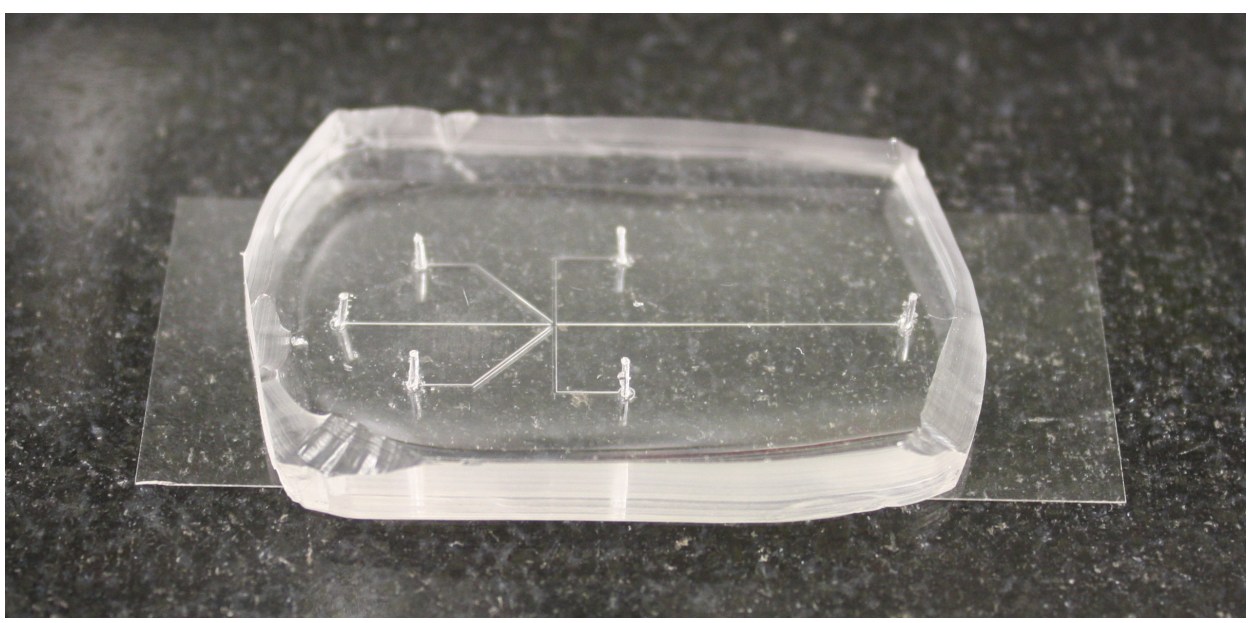

(c)

Figure 3.6: Images of the microfluidic device. (a) Image of the PDMS slab that is used to produce the NOA step structure. (b) Image of the PDMS slab with punched holes. (c) Image of the final PDMS-glass device. 


\subsubsection{Experimental setup}

The sample fluids are applied to the microfluidic device with gastight syringes (volume $100 \mu l, 250 \mu l, 500 \mu l$, Hamilton, Bonaduz, GR, Switzerland) connected with polyethylene tubing. The syringes are driven with high precision syringe pumps (Nemesys, Cetoni, Korbußen). The tubings for connecting the syringes to the device have a inner volume of $\sim 40 \mu l$. To use the full volume of the syringes, the tubings are filled with solution from another syringe before connecting the syringe used for the experiment.

\subsubsection{Surface modifications}

PLL-g-PEG For the surface treatment with PLL-g-PEG we use PLL(20)-g[3.5]-PEG(2) (SuSoS, Dübendorf, Switzerland). We flush a solution of $1 \mathrm{~g} / 1 \mathrm{PLL}-\mathrm{g}-\mathrm{PEG}$ in $2 \mathrm{mM}$ phosphate buffer, pH 7.5 through the freshly plasma cleaned and assembled microfluidic device for 20 minutes. The PLL-g-PEG covers the surface with a uniform layer. For the long term stability that is needed for the assembly experiments (several hours), the anti-stick properties are not sufficient. In the assembly experiments, the protein adsorbs to the wall after coming into contact with the salt ions that trigger the assembly.

Poloxamer 407 For the coating with Poloxamer 407, a solution with $0.2 \%$ Poloxamer 407 (Sigma-Aldrich, St. Louis, Missouri, USA) is flushed through the assembled mixing device for two hours. The blocking properties are not sufficient for the assembly experiments. The protein still adsorbs to the channel walls where it comes into contact with the salt ions.

BSA Bovine serum albumin (BSA) is dissolved at a concentration of $1 \mathrm{mg} / \mathrm{ml}$ in $2 \mathrm{mM}$ phosphate buffer at $\mathrm{pH}$ 7.5. The device is flushed with the BSA solution for 30 minutes. BSA adsorbs to the channel surfaces and forms a protective layer. The surface treatment with BSA does not prevent the adsorption of assembling vimentin for a time long enough to perform the assembly experiments in the microfluidic flow device.

Lipid coatings Dried lipids were provided by Hannes Witt (Department for Biophysical Chemistry, University of Göttingen). To produce unilamelar vesicles, the dried lipids are dissolved in $2 \mathrm{mM}$ phosphate buffer, $\mathrm{pH}$ 7.5. The solution with the dissolved lipids is sonicated with a probe sonicator (SonoPuls UW 2070, Bandelin, Berlin, Germany) 


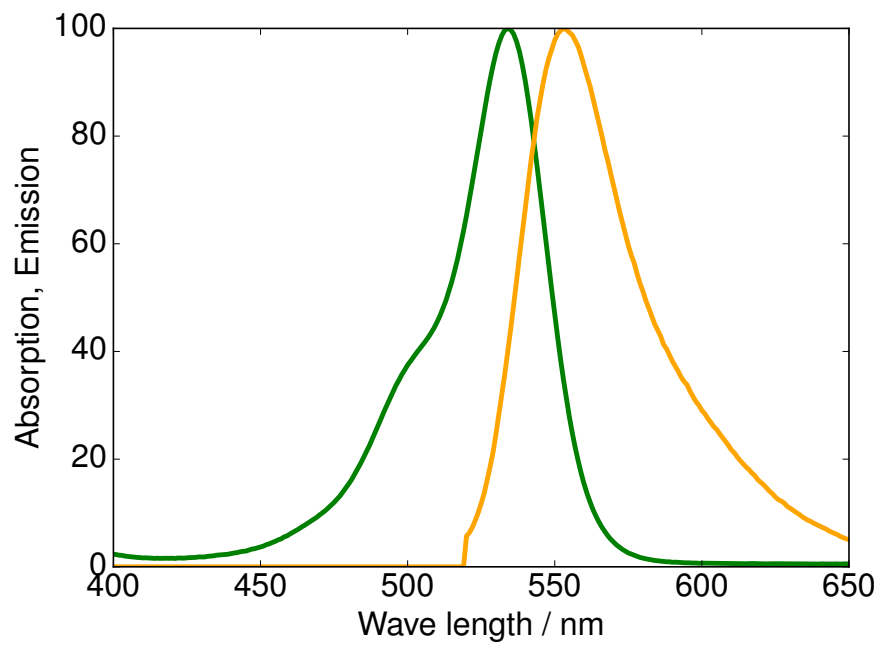

Figure 3.7: Absorption and emission spectra for the dye Alexa Fluor 532. Absorption is shown in green, emission is shown in orange. Data from [69].

for 20 minutes at a $10 \%$ duty cycle and $50 \%$ intensity. The reaction tube is placed in a water bath to avoid heating of the solution. The solution is then centrifuged at $12100 \mathrm{~g}$ for 30 minutes to remove titanium particles from the probe sonicator. The sediment is discarded. The microfluidic device is flushed with the lipid vesicles for 30 minutes.

\subsection{Microscopy techniques}

\section{Epi-fluorescence microscopy}

In epi-fluorescence microscopy the sample is illuminated with light of specific wavelength (see Figure 3.8). The light of a mercury arc lamp (X-Cite 120 PC Q, Excelitas Technologies) is guided onto a fluorescence filter cube, which selects the wavelength for excitation. The filtered light is guided towards the objective. The fluorescent light that is emitted by the sample is collected by the same objective and guided through the filter cube towards the camera or ocular. Images are acquired with a CCD-camera (Hamamatsu Orca R-2, Hamamatsu Photonics Deutschland GmbH, Herrsching am Ammersee, Germany) controlled by the software Micro-Manager 68]. 


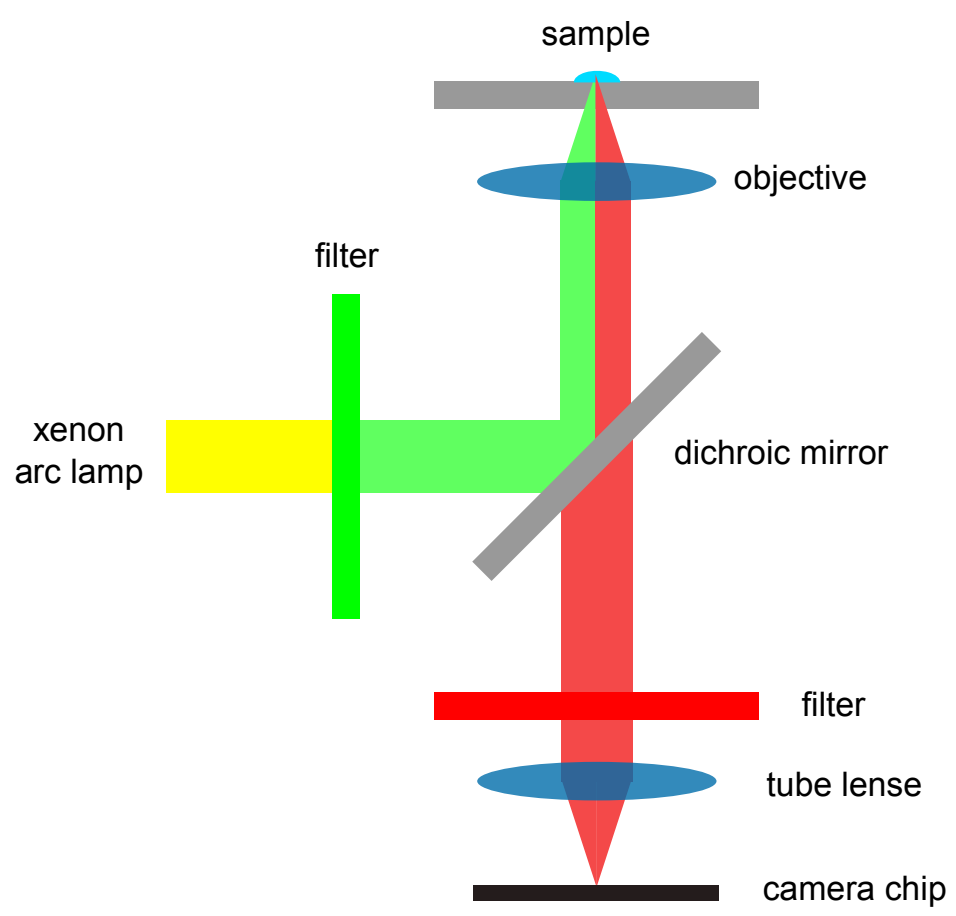

Figure 3.8: Lightpath of an epi-fluorescence microscope. The fluorescently labeled sample is illuminated through the objective with light of a specific wavelength and the emitted fluorescent light is collected by the same objective. The emitted light passes through the emission filters to block excitation light. The fluorescent signal is detected on a camera chip. 
3 Materials and methods

\section{Confocal microscopy}

In confocal microscopy, a focused laser beam is scanned across the sample and the fluorescence light is recorded. Off-axis light is blocked by a pinhole. We use a Olympus IX 81

microscope with the FV1000 extension. Images are analyzed with the software package Fiji 70]. 


\section{Results}

In this chapter, we will first describe the FCS setup that was built for this thesis project. Then, the results of the vimentin labeling procedure and the results of the vimentin assembly experiments in bulk are presented. Subsequently, the microfluidic device and the results of the vimentin assembly experiments in microfluidic flow are presented.

\subsection{Construction of the FCS setup}

The FCS setup was planned and built during this thesis project. The basic components of the setup - including the microscope body, laser source, photo detectors and the data acquisition card - was proposed by Bernd Nöding.

\subsubsection{Hardware setup}

The FCS setup is based on a Olympus IX 73 inverted microscope (Olympus, Hamburg, Germany). A schematic of the hardware setup is shown in Figure 4.1. The light path in the microscope is accessed via a side port in one of the filter turret slots (see Figure 4.3). The light of the continuous wave excitation laser source (Cobolt Samba 25, Cobolt AB, Solna, Sweden) with a wavelength of $532 \mathrm{~nm}$ and a maximum power of $25 \mathrm{~mW}$ is coupled into a single mode fiber (kineFLEX, Qioptiq Photonics GmbH \& Co KG, Göttingen). The single mode fiber spatially filters the light to provide a Gaussian beam profile. After exiting the single mode fiber, the laser beam has a diameter of $0.7 \mathrm{~mm}$ and is guided through a laser clean up filter which filters out light with a wavelength deviating from the main laser wavelength (HC Laser Cleanup 532/2, AHF, Tübingen). Figure 4.2 shows the spectra of the fluorescent dye ATTO532 and the spectra of the laser clean up filter and the main dichroic mirror. The laser beam is focused onto a $50 \mu \mathrm{m}$ pinhole (Qioptiq, Göttingen) by a lens with a focal length of $f=20 \mathrm{~mm}$ and is subsequently re-collimated with a $f=200 \mathrm{~mm}$ lens in order to clean up the beam. This is done in order to clean 


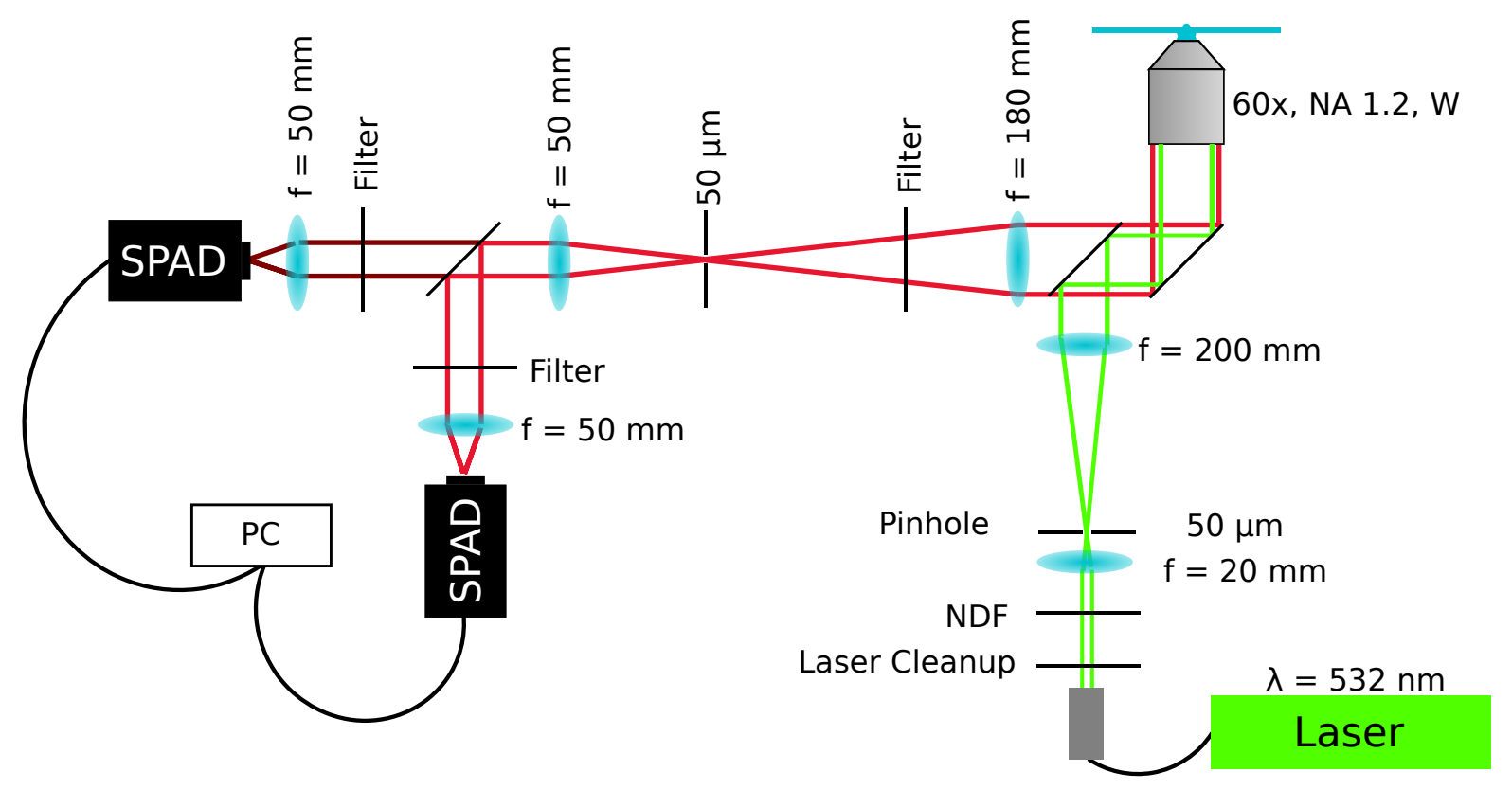

Figure 4.1: Beam path of the FCS setup. A laser beam is widened and guided onto the sample. The fluorescent light from the sample is collected and focused onto a pinhole. The signal is detected with single photon avalanche diodes and correlated with a hardware correlator. NDF - neutral density filter.

up the beam, in addition to the filtering by the single mode fiber, and to expand the beam width by a factor of 10. All lenses are purchased from Qioptiq Photonics GmbH \& Co. KG, Göttingen, unless stated otherwise. The laser beam is expanded in order to illuminate the full back aperture of the objective (diameter $d=7 \mathrm{~mm}$ ). We use an Olympus UPLSAPO 60XW water immersion objective with a numerical aperture of N.A. $=1.2$. The intensity of the laser is attenuated with neutral density filters, and the laser power at the objective is measured with a power meter (PM120D, Thorlabs $\mathrm{GmbH}$, Dachau/Munich). The laser power used for the experiments lies between $10 \mu \mathrm{W}$ and $20 \mu \mathrm{W}$. The widened beam is then reflected towards the microscope body by a dichroic mirror with high reflectivity at $532 \mathrm{~nm}$ (Laser-Beamsplitter 532, AHF, Tübingen). The emitted fluorescent light is collected with the same objective and is guided back through the same dichroic mirror. The excitation light is blocked in the emission beam path with a filter (RazorEdge LP 532, AHF, Tübingen). A $f=180 \mathrm{~mm}$ lens (AC508-180, Thorlabs GmbH, Dachau/Munich) focuses the emission light onto a pinhole with a radius of $r_{\text {pin }}=25 \mu \mathrm{m}$. The pinhole blocks the part of the emission light that is not coming from the focus of the excitation laser spot. The emission light is 


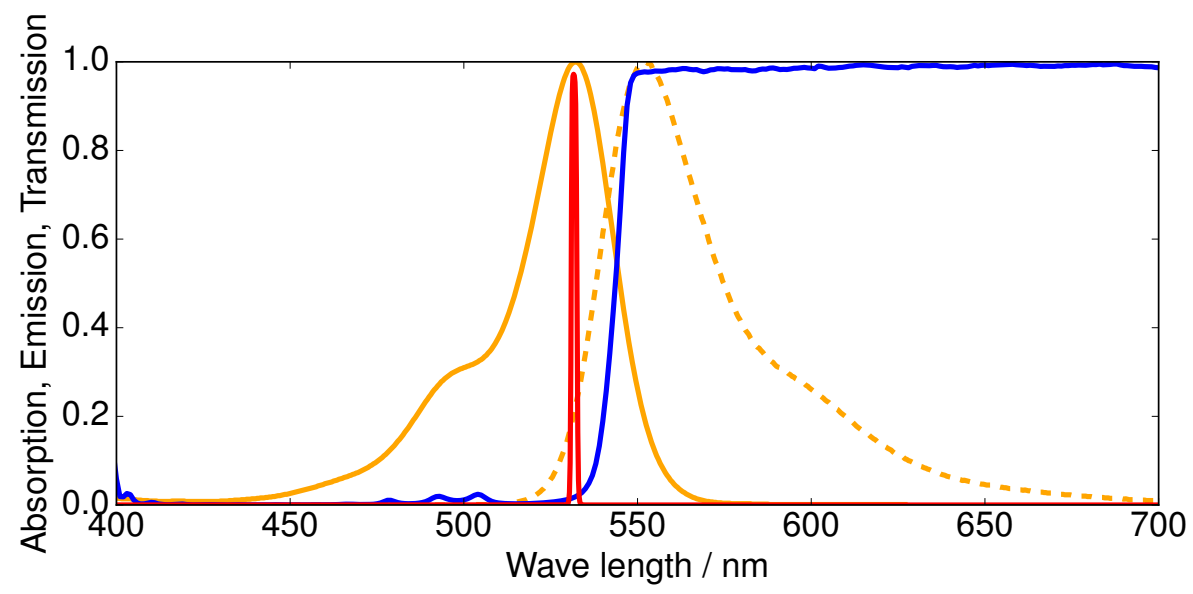

Figure 4.2: Spectra of ATTO532 and the fluorescence filters. Excitation (solid yellow line) and emission (dotted yellow line) spectra of ATTO532. Transmission spectrum of the main dichroic mirror (blue) and the laser clean up filter (red). Data from 7173

re-collimated with a $f=50 \mathrm{~mm}$ lens.

A filter cube guides the emission light into two directions. In both directions, lenses with $f=50 \mathrm{~mm}$ focus the light onto the active area (diameter $150 \mu \mathrm{m}$ ) of two singlephoton avalanche diodes (SPADs) with a maximum dark count rate of 20 counts per second ( $\tau$-SPAD, Picoquant GmbH, Berlin) for the detection of the fluorescent signal. The SPADs are connected to a hardware correlator (ALV-7004 USB, ALV-Laser Vertriebsgesellschaft $\mathrm{mbH}$, Langen). The hardware correlator calculates four correlation functions simultaneously: the auto-correlation functions of the individual signals of the two SPADs (ACF 0 and ACF 1), and the forward and backward cross-correlation functions between the two detector signals (CCF 0/1, CCF 1/0). With a 50:50 beam splitter placed in the filter cube before the photon detectors, the light is split independent of the wavelength. In this configuration, the cross-correlation between the two detectors estimates the ACF. This configuration has the advantage that the resulting curve is not influenced by the afterpulsing effect (see Section 16). The 50:50 beam splitter is used for the experiments in the following sections.

With a dichroic mirror in front of the photon detectors, the fluorescent light is split depending on the wavelength. This allows for calculating the cross-correlation of two distinct fluorescent dyes as used for two-color FCCS. With the single excitation laser configuration, the setup is suitable for single wavelength FCCS (SW-FCCS) [59]. For an 


\section{Results}

efficient detection of the fluorescent signal, we optimized the length of the beam path outside of the microscope body.

The ALV correlator additionally acquires the count rate traces for the two detectors with a sampling rate of $25 \mathrm{~Hz}$. This trace allows for detecting the transition of single bright particles through the focal volume. However, the sampling rate is too low for the analysis of the photon data. To access the raw photon arrival times we use a separate data acquisition card (NI PCI-6602, National Instruments, Austin, USA) and a customwritten program that builds on a python interface for the data acquisition card [74]. The photon arrival times are measured relative to an arbitrary start point. For acquisitions where only few photons per interval are detected, this mode is advantegeous. In this mode, the amount of data is lower than for the acquisition of photons per time interval. The acquired photon arrival times can be used for further analysis with methods like photon counting histogram or burst analysis.

For the measurements in microfluidic channels (see Section 4.4), we need to access different areas of the sample. For this, we use a automated sample stage (Prior Scientific Inc., Rockland, MA, USA). The stage is controlled via the ALV data acquisition software and is programmed to move along a pre-defined grid. At every point an FCS measurement is taken.

Short, individual measurements $(\approx 10 \mathrm{~s})$ are taken to be able to sort out measurements with perturbations caused by, for example, bright clusters. Single bright events can affect the curves strongly. These curves are characterized by not decreasing to zero for long lag times and are excluded from further analysis. For FCS measurements, data are analyzed with the program Quickfit 3.0 [75]. For measurements of diffusing molecules in three dimensions, the following fit function is used (see Section 2.4.1):

$$
G_{3 \mathrm{D}}(\tau)=\frac{1}{N} \cdot \frac{1}{1+\tau / \tau_{\text {diff }}} \sqrt{\frac{1}{1+\tau /\left(\gamma^{2} \tau_{\text {diff }}\right)}}
$$

For measurements with flow, the following fit function is used:

$$
G_{3 \mathrm{D}, \text { flow }}(\tau)=G_{3 \mathrm{D}}(\tau) \cdot \exp \left(-\left(\frac{\tau}{\tau_{\text {flow }}}\right)^{2} \cdot\left(1+\frac{\tau}{\tau_{\text {diff }}}\right)^{-1}\right)
$$




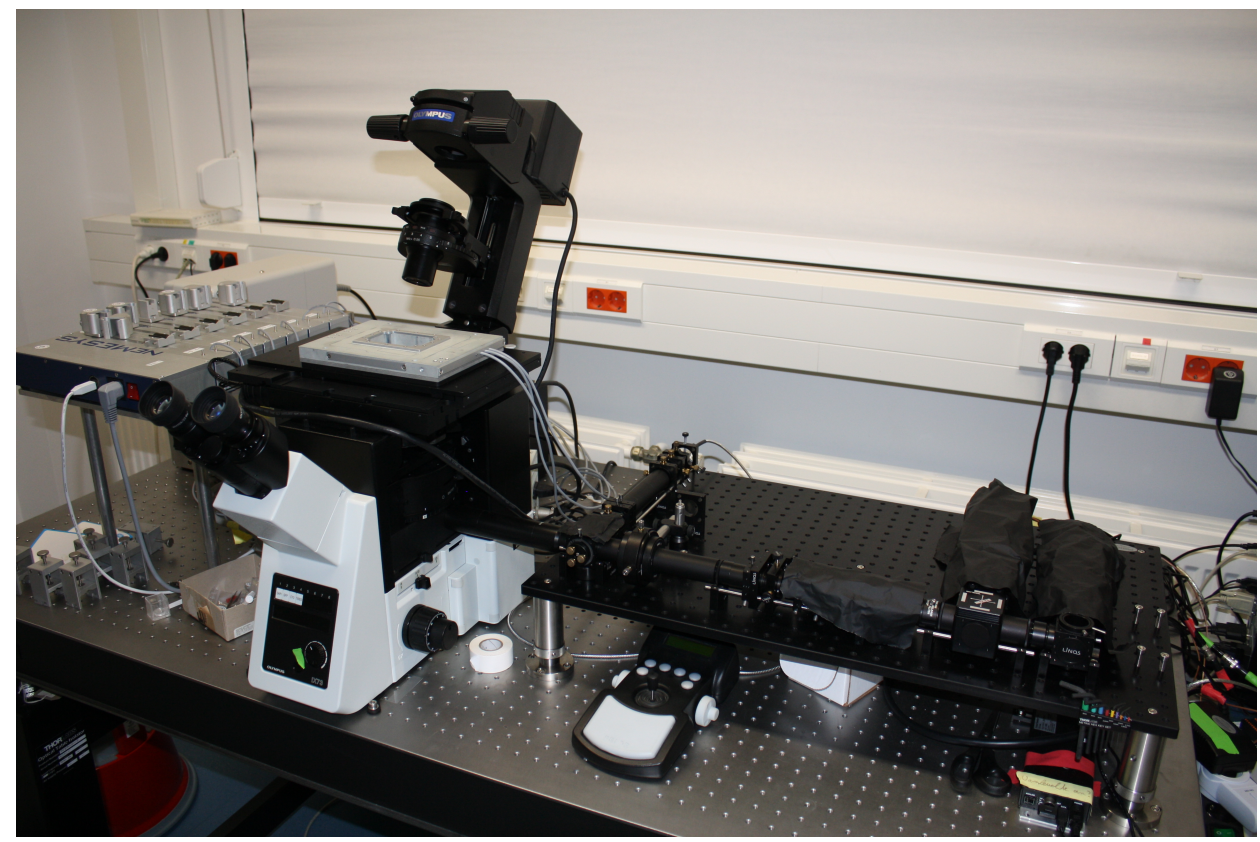

(a)

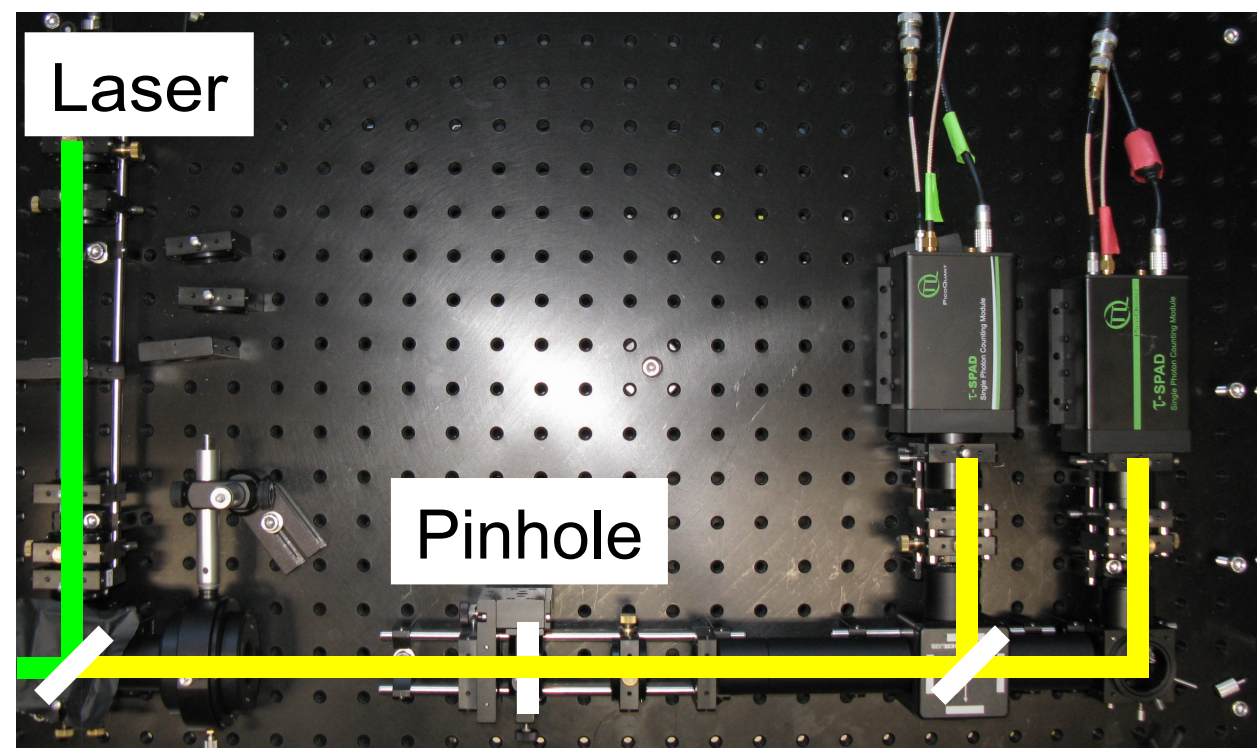

(b)

Figure 4.3: Images of the FCS setup. (a) Overview image of the whole setup. (b) The image shows the optical beam path and the two photon counting modules on the right side. The green ray leads to the microscope to the left side. 


\subsubsection{Calibration of the setup}

For determining absolute values for the diffusion coefficient, the FCS setup needs to be calibrated. The FCS measurements yield only diffusion times, but when the detection volume is known, absolute diffusion coefficients can be calculated. For this, we need to determine the size of the detection volume. This is achieved by measuring the diffusion time of a molecule with a known diffusion coefficient. We use the fluorescent dye Rhodamine 6G (Thermo Fisher, R634) dissolved in deionized water. The diffusion coefficient of this dye was determined with dual-focus FCCS to be $D_{25}{ }^{\circ} \mathrm{C}=(414 \pm 5) \mu \mathrm{m}^{2} / \mathrm{s}[52$. The diffusion coefficient depends on the temperature. At the temperature of $21^{\circ} \mathrm{C}$ in our lab, this corresponds to $D_{21}{ }^{\circ} \mathrm{C}=372 \mu \mathrm{m}^{2} / \mathrm{s}$. When the diffusion coefficient $D$ and the diffusion time $\tau_{\text {diff }}$ are known, the radius of the detection volume $w_{\mathrm{xy}}$ is calculated with the following equation

$$
w_{\mathrm{xy}}=\sqrt{4 D \tau_{\text {diff }}} .
$$

For Rhodamine $6 \mathrm{G}$ we measure a diffusion time of $45 \mu \mathrm{s}$. Thus, the calibration with Rhodamine $6 \mathrm{G}$ yields for the detection volume (see Figure 4.4):

$$
w_{\mathrm{xy}}=(260 \pm 10) \mathrm{nm} .
$$

At a laser power at the objective of $P=12 \mu \mathrm{W}$, we measure a count rate per molecule of $45 \mathrm{kHz}$. The count rate per molecule is calculated by dividing the total count rate by the number of molecules $N$ in the focus volume. The number of molecules $N$ is obtained from the fit of the ACF.

\subsection{Vimentin labeling}

The labeling procedure is described in Section 3.1.1. In short, vimentin molecules are fluorescently labeled via a maleimide reaction and purified by size exclusion chromatography. Figure 4.5 shows assembled vimentin filaments with fluorescence microscopy. Here, we show the outcome of the labeling procedure. 


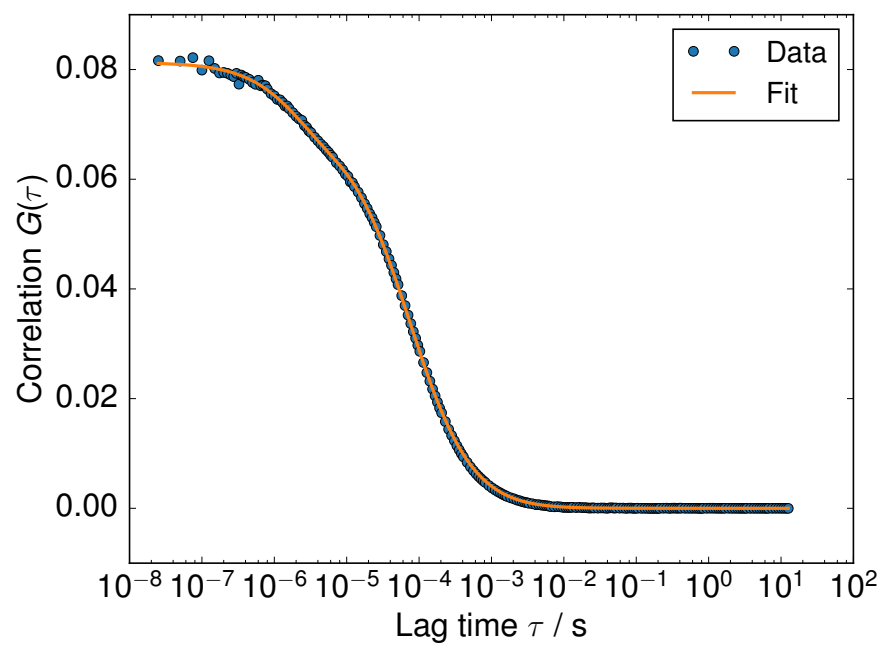

Figure 4.4: FCS curve for Rhodamine 6G. For the calibration of the setup we measured a solution containing $38 \mathrm{nM}$ of Rhodamine 6G. CCF with 50:50 beam splitter. The fit contains a triplet component.

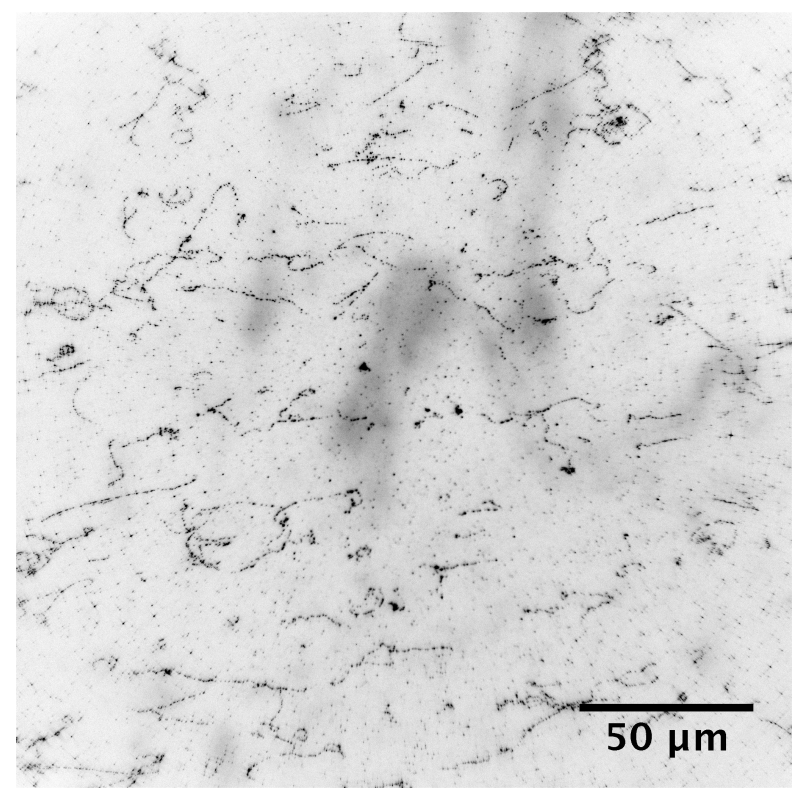

Figure 4.5: Fluorescence image of assembled vimentin filaments. Vimentin filaments were assembled for 24 hours. The filaments appear spotted due to the sparse amount of labeled monomers. 


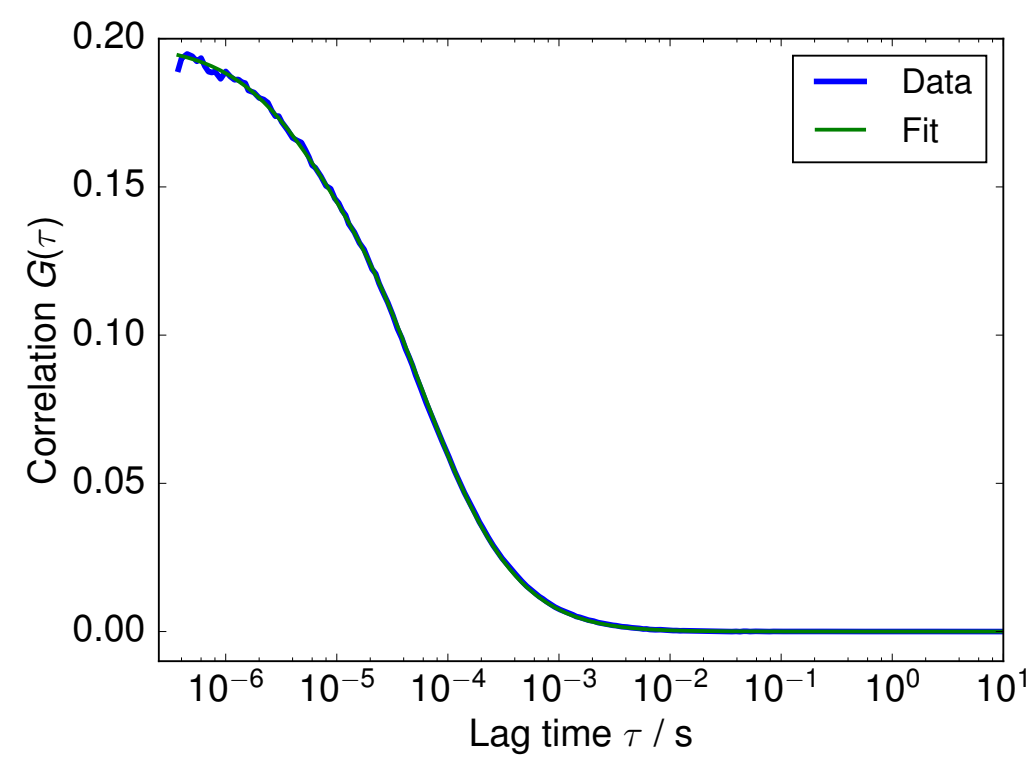

Figure 4.6: FCS curve for Atto 532. The fit yields a value of $D=320 \mu \mathrm{m}^{2} / \mathrm{s}$ for Atto 532.

\subsubsection{Detection of free dye}

After the labeling procedure the solution contains labeled vimentin and free dye molecules. Solutions of labeled vimentin are only fitted well when we assume several diffusing species. We measure the diffusion coefficient of free Atto 532 molecules to be $D_{\text {Atto532 }}=$ $320 \mathrm{\mu m}^{2} / \mathrm{s}$ (see Figure 4.6). The FCS curve for labeled vimentin is fitted with a twocomponent fit function:

$$
G_{3 \mathrm{D}, \mathrm{comp} 2}(\tau)=\frac{1}{N}\left(\rho_{1} \cdot g_{1}(\tau)+\left(1-\rho_{1}\right) \cdot g_{2}(\tau)\right)
$$

where

$$
g_{i}(\tau)=\frac{1}{1+\tau / \tau_{\text {diff }, i}} \sqrt{\frac{1}{1+\tau /\left(\gamma^{2} \tau_{\text {diff }, i}\right)}} .
$$

For the fit procedure the diffusion coefficient of the first component is set to the value of free Atto 532 dye $\left(D=320 \mu^{2} / \mathrm{s}\right.$, see Figure 4.7). The second component is then assumed to be the labeled vimentin molecules. For unassembled vimentin we measure a diffusion coefficient of $D_{\mathrm{Vim}, 20 \mathrm{~W}}=(25 \pm 2) \mu \mathrm{m}^{2} / \mathrm{s}$. 
4.2 Vimentin labeling

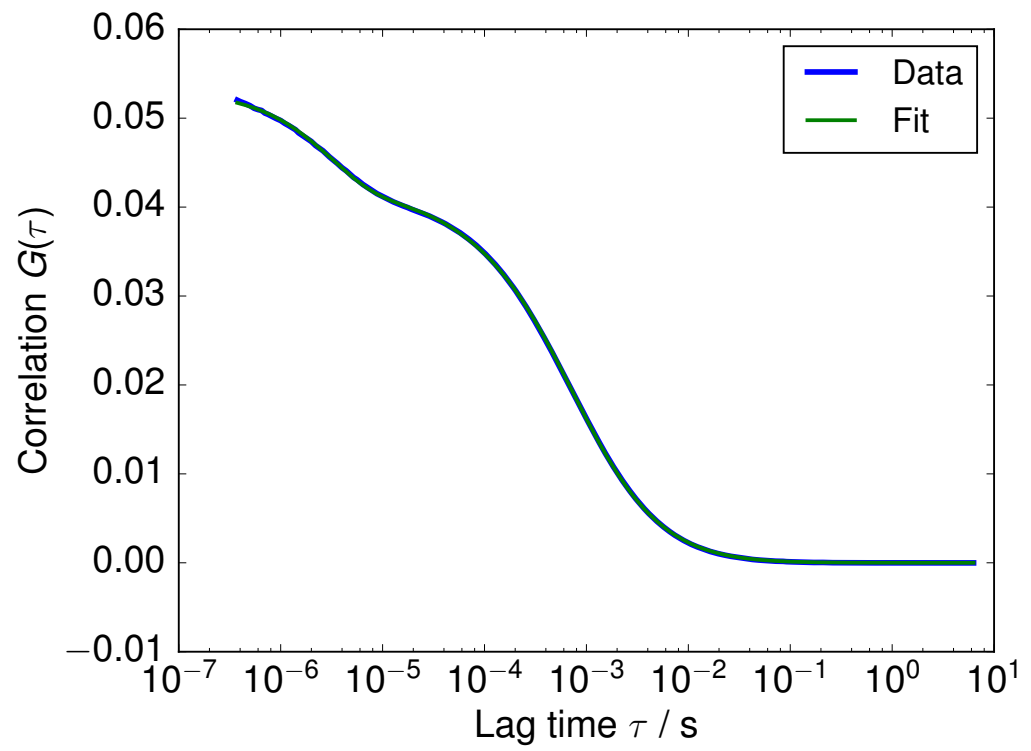

Figure 4.7: FCS measurement of vimentin labeled with Atto 532. A twocomponent fit is used where one component is fixed at $D=320 \mu^{2} / \mathrm{s}$ for free Atto 532, and the other component is vimentin tetramers with: $D_{\text {Vim }, 20 \mathrm{~W}}=(25 \pm 2) \mu \mathrm{m}^{2} / \mathrm{s}$. The fit accounts for the triplet state dynamics visible at a lag times around $10^{-6}$ seconds. 


\subsubsection{Separation of labeled vimentin and free dye}

For the separation of the labeled vimentin and the free dye we tested several procedures.

Before the assembly experiments the protein needs to be dialyzed from $8 \mathrm{M}$ urea to $2 \mathrm{mM}$ phosphate buffer. The dialysis tubing has a molecular weight cut-off of $50 \mathrm{kDa}$, and the free dye molecules have a molecular weight of around $1 \mathrm{kDa}$. Therefore, the free dye molecules are able to diffuse out of the protein solution. We extended the dialysis time to several days and checked for the free dye content with FCS measurements, but a significant decrease in the free dye content could not be detected. We also used size exclusion columns of different length and found that the amount of free dye can be decreased by using a longer column. 
4.3 Vimentin assembly in bulk

\subsection{Vimentin assembly in bulk}

With vimentin assembly 'in bulk' we describe the experiments in a static volume, in contrast to the experiments in a microfluidic mixer that will be described in Section 4.5 . For vimentin assembly in bulk we employ two different routines that we describe as the 'continuous method' and the 'reaction tube method'. In both routines we start the assembly process by mixing dialyzed vimentin $1: 1$ with phosphate buffer that contains $\mathrm{KCl}$ in the specific concentrations for the experiments (for vimentin dialysis see Section 3.1.3). The solution then is incubated at $22^{\circ} \mathrm{C}$ or $37^{\circ} \mathrm{C}$ and measured with one of the two routines.

In the continuous method we record data at $22^{\circ} \mathrm{C}$. After starting the assembly process, we place the protein solution directly on the sample holder of the FCS setup and measure the sample continuously with data acquisitions of 10 seconds. Contrary to the reaction tube method (see below) the sample is not diluted, so that we measure at the same vimentin concentration at which the assembly process was started.

To check for the temporal stability of the vimentin FCS measurement, we measure unassembled vimentin with the continuous method. Figure 4.8 shows the resulting graphs for fluorescent intensity, diffusion coefficient, particle concentration in focus and count rate per molecule. For the diffusion coefficient we find a value of around $25 \mathrm{\mu m}^{2} / \mathrm{s}$ which is the expected value for vimentin tetramers. All graphs show only small fluctuations over the measurement time of four hours. The errors of the evaluation are determined from the variance-covariance matrix of the least-squares fit. We assume the experiment to be stable enough so that we can attribute large changes to the assembly process. The countrate per molecule is around $40 \mathrm{kHz}$.

Figure 4.9 shows the assembly of vimentin at $c_{\mathrm{vim}}=200 \mathrm{mg} / 1, c_{\mathrm{KCl}}=100 \mathrm{mM}$ and a temperature of $T=22{ }^{\circ} \mathrm{C}$. The assembly is measured over 240 minutes. During this time, the count rate is decreasing slightly. The large peak in the beginning occured during the pipetting of the solution onto the cover slide. The diffusion coefficient shows a decrease from $20 \mathrm{\mu m}^{2} / \mathrm{s}$ to $7 \mathrm{\mu m}^{2} / \mathrm{s}$, indicating an increase in the particle size. The particle concentration is decreasing over time, while the count rate per molecule is ascending. This corresponds to the binding of labeled molecules, which is expected for the assembly process. The particle concentration in focus and the count rate per molecule show similar fluctuations. This could indicate that the fluctuations in the count rate trace are caused by fluctuations of the particle concentration. The fluctuations in the 


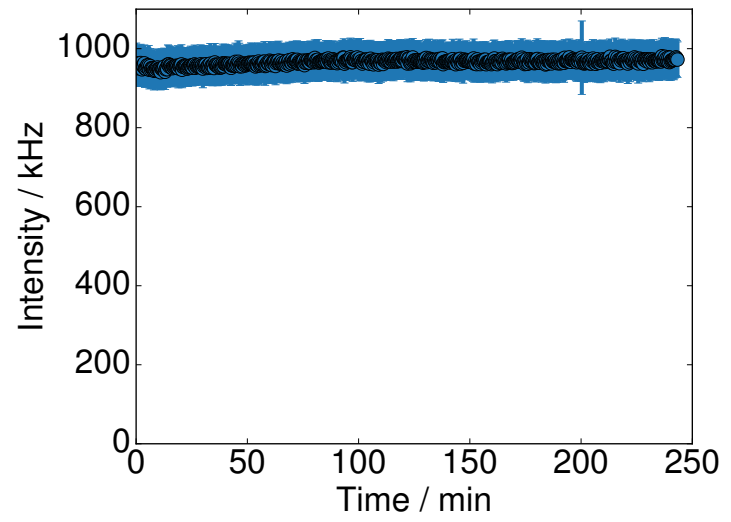

(a)

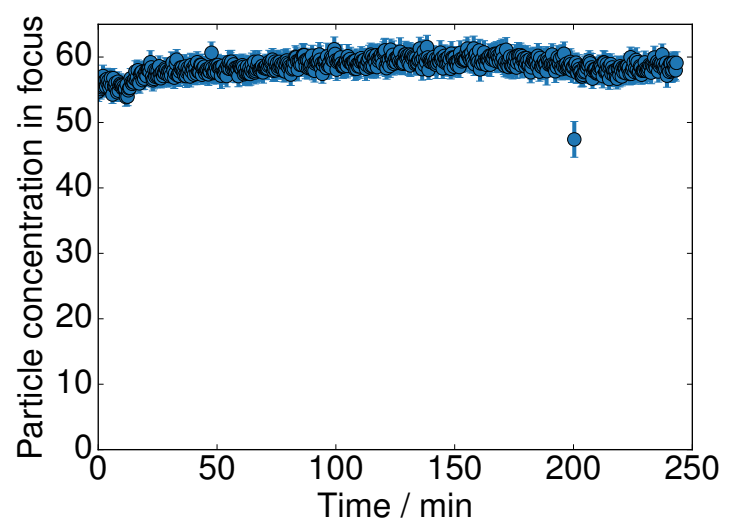

(c)

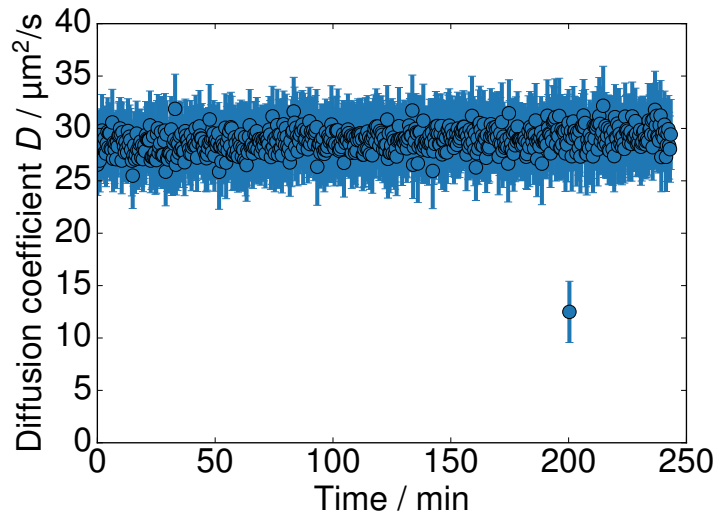

(b)

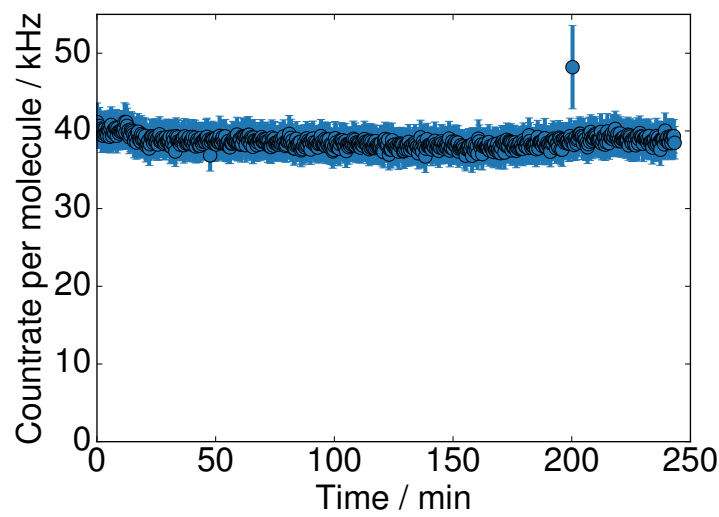

(d)

Figure 4.8: Unassembled vimentin measured in continuous mode. The protein concentration is $200 \mathrm{mg} / \mathrm{l}$. (a) Fluorescent intensity. (b) Diffusion coefficient. (c) Particle concentration in focus. (d) Count rate per molecule. 
particle concentration might be due to drift. 


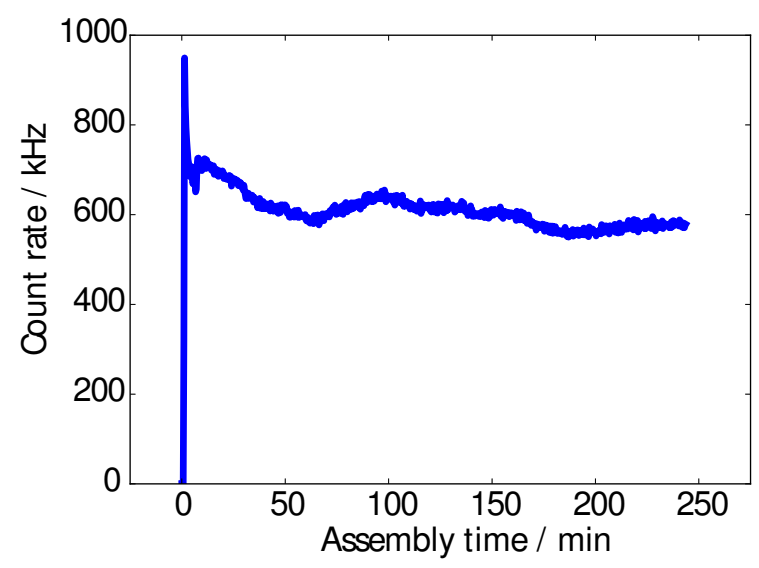

(a)

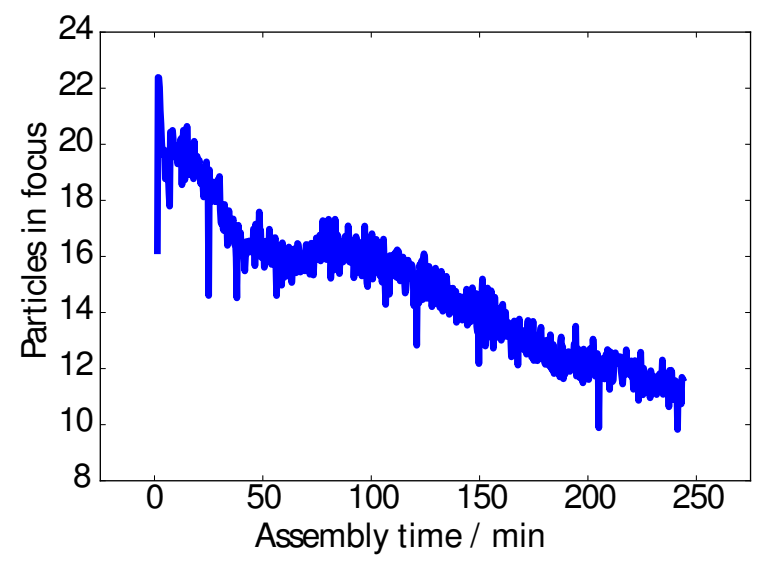

(c)

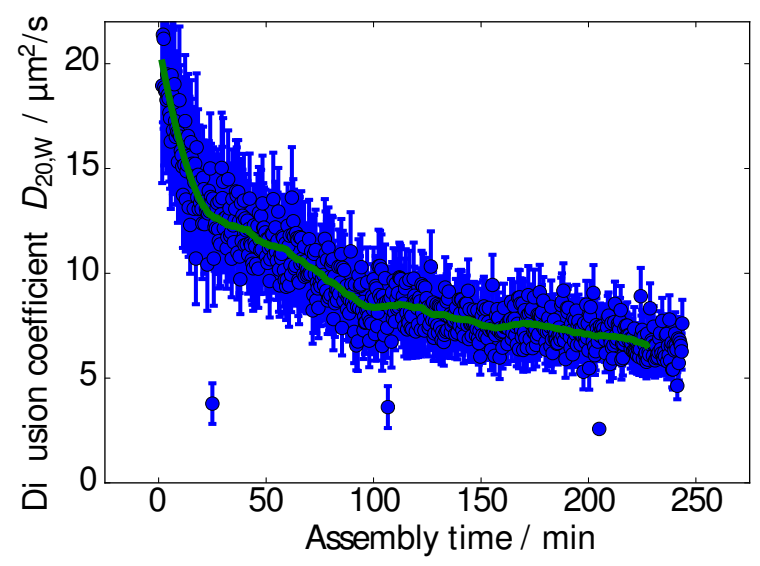

(b)

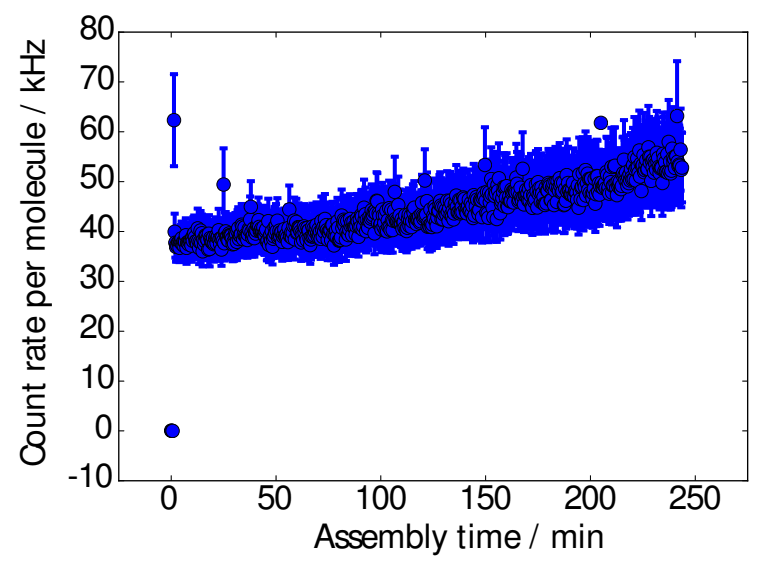

(d)

Figure 4.9: Vimentin assembly measured in continuous mode. The protein concentration is $c_{\text {Vim }}=200 \mathrm{mg} / 1, c_{\mathrm{KCl}}=100 \mathrm{mM}$, temperature $T=22^{\circ} \mathrm{C}$. (a) Count rate. (b) Diffusion coefficient. (c) Particle concentration in focus. (d) Count rate per molecule. 


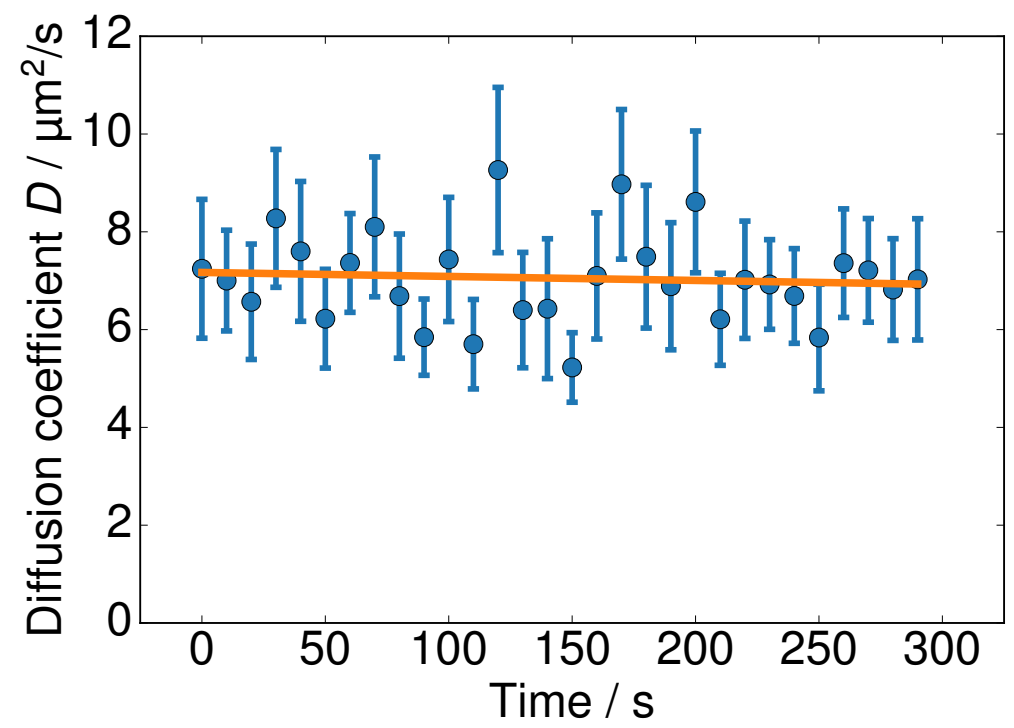

Figure 4.10: Diffusion coefficient of vimentin diluted after one minute of assembly. Blue - Data points, Orange - Linear regression. Vimentin is assembled for one minute at $200 \mathrm{mg} / \mathrm{l}$ at $37^{\circ} \mathrm{C}$ and $100 \mathrm{mM} \mathrm{KCl}$, then diluted to $20 \mathrm{mg} / \mathrm{l}$. The linear regression shows a slight decrease over the time course of one measurement (slope $p=-8 \cdot 10^{-4}$ ).

In the reaction tube method the solution is incubated at $22^{\circ} \mathrm{C}$ and $37^{\circ} \mathrm{C}$. During the incubation we take samples after 1, 3, 5, and 10 minutes and then in steps of 10 minutes until a total time of 60 minutes. We immediately dilute the samples $1: 10$ maintaining the $\mathrm{KCl}$ concentration after taking the samples from the incubation solution. We measure every sample in total for 5 minutes and determine the diffusion coefficient from the fit. Each measurement is divided into 30 acquisitions of 10 seconds each.

To check how the assembly continues during this measurement, we assemble vimentin for one minute at a temperature of $37^{\circ} \mathrm{C}$, a $\mathrm{KCl}$ concentration of $100 \mathrm{mM}$ and a protein concentration of $200 \mathrm{mg} / \mathrm{l}$. After dilution to $20 \mathrm{mg} / \mathrm{l}$ the sample is measured (see Figure 4.10p. The linear regression shows a slight decrease over time. This decrease is typically smaller than the difference between the samples of different assembly times. Thus we can use the averaged value of 5 minutes for the representation in the assembly graph over 60 minutes. 


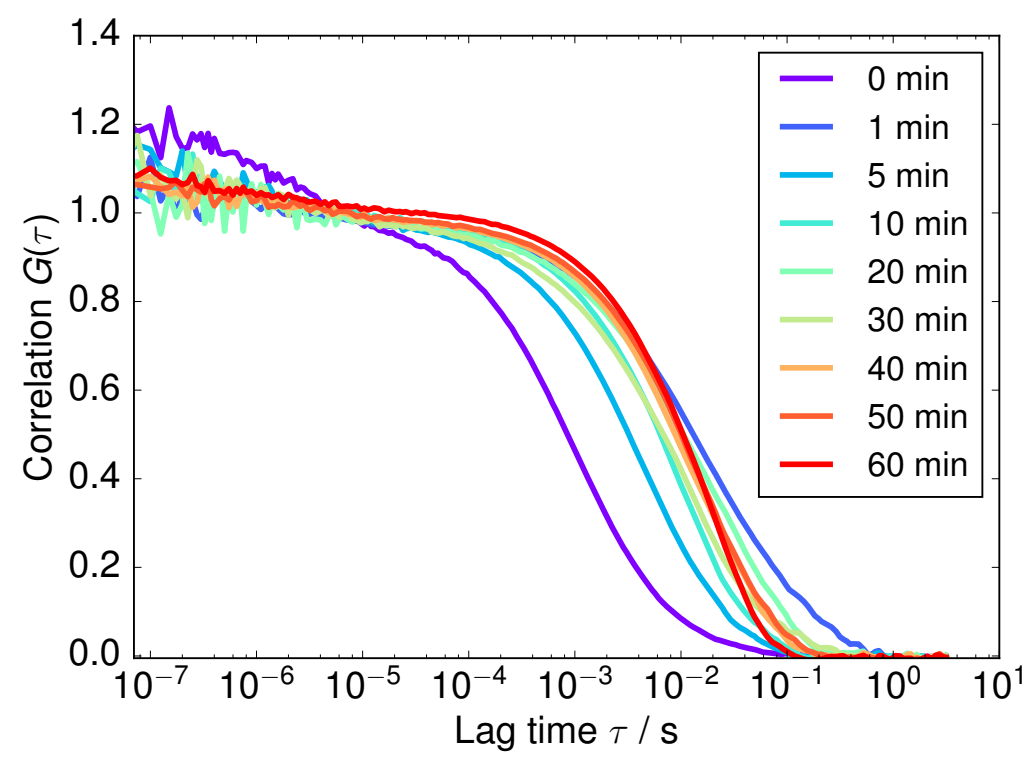

Figure 4.11: Overlay of FCS curves for vimentin assembly. Vimentin was assembled at $37^{\circ} \mathrm{C}$ at a concentration of $50 \mathrm{mg} / \mathrm{l}$ by adding salt to a final concentration of $100 \mathrm{mM} \mathrm{KCl}$. Samples were taken from the solution at the indicated time points, diluted $1: 10$ and measured.

An overlay of all curves from a typical measurement for the reaction tube method is shown in Figure 4.11. Vimentin was assembled at $37^{\circ} \mathrm{C}$ at a concentration of $50 \mathrm{mg} / \mathrm{l}$ by adding salt to a final concentration of $100 \mathrm{mM} \mathrm{KCl}$. The curves are normalized to the same particle number. The triplet part is not taken into account for the normalization. The curves shift to the right with increasing assembly time, indicating a decrease in particle size. The largest shift occurs between the first and the second curve, corresponding to $0 \mathrm{~min}$ and $1 \mathrm{~min}$. To compare the two methods described above we perform experiments with the same parameters (see Figure 4.12). The two methods show a similar behavior for the decreasing diffusion coefficient. The diffusion coefficient decreases to a value around $10 \mathrm{\mu m}^{2} / \mathrm{s}$ after 60 minutes. 


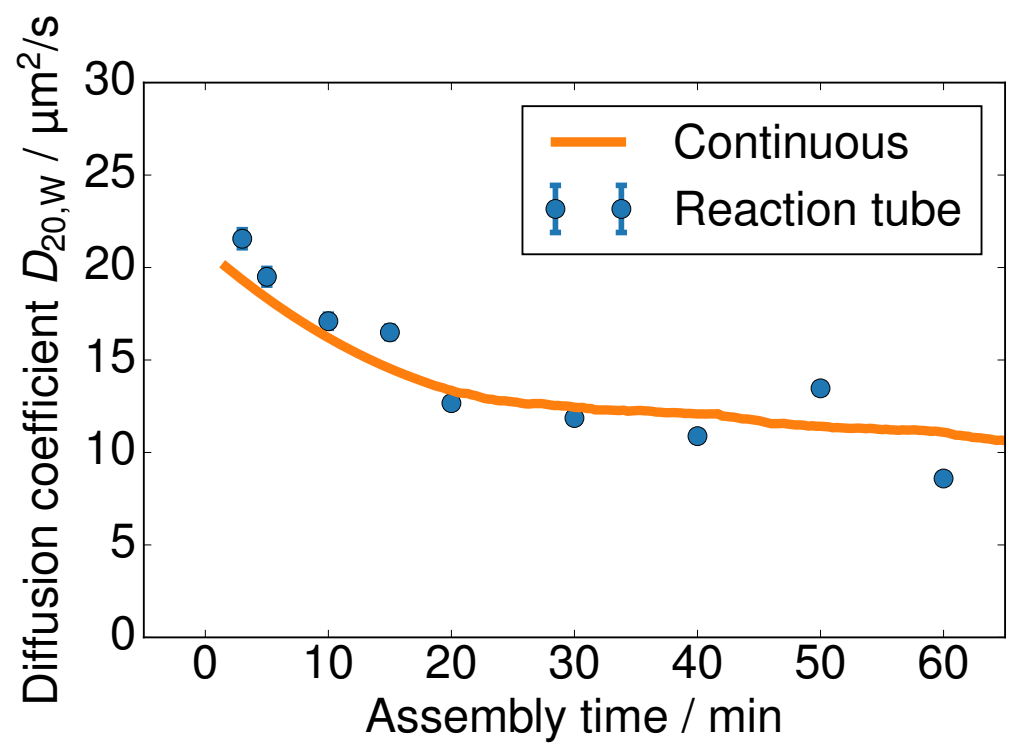

Figure 4.12: Vimentin assembly in the continuous mode and in the reaction tube mode. For the reaction tube experiment, samples from the assembly solution were taken at several time points and diluted to slow down assembly. In the continuous experiment, the assembly solution was measured continuously. The experiments were performed with a vimentin concentration of $200 \mathrm{mg} / \mathrm{l}$ and a $\mathrm{KCl}$ concentration of $100 \mathrm{mM}$ at a temperature of $22{ }^{\circ} \mathrm{C}$. The decrease of the diffusion coefficient shows a similar behavior for both curves. 


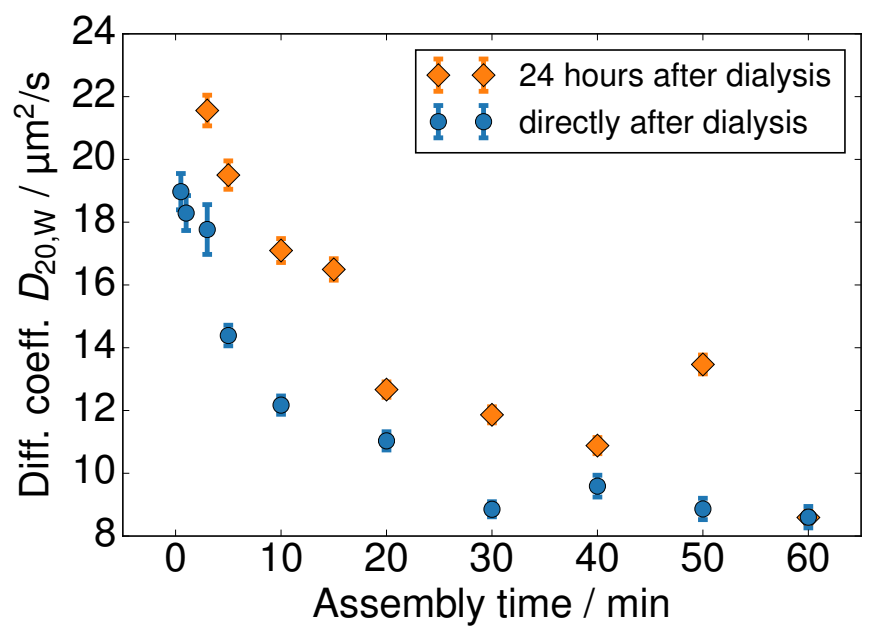

Figure 4.13: Dependence between storage time after dialysis and the ensuing vimentin assembly. Vimentin assembly experiments were carried out at different time spans after the dialysis of the protein at $c_{\text {Vim }}=200 \mathrm{mg} / \mathrm{l}$, $c_{\mathrm{KCl}}=100 \mathrm{mM}$, and temperature $T=22^{\circ} \mathrm{C}$. The assembly tends to slow down for protein that was stored for a longer time after dialysis.

The vimentin is dialyzed from storage buffer to phosphate buffer. After the last dialysis step overnight the protein is stored at $10^{\circ} \mathrm{C}$. We took measurements of the assembling vimentin protein directly after dialysis and after storing it for 24 hours. Each curve is the measurement of one experiment. This might explain the large variations in the single curves. We noticed that the change in diffusion coefficient depends on the time the vimentin solution is stored before the experiment is carried out (see Figure 4.13). The protein that was stored for a longer time before the experiment assembles more slowly than right after dialysis. This behavior might be caused by protein degradation. The number of functional tetramers is lower and assembly is slowed down. From this we conclude that the time between the end of the dialysis and the experiment alters the results.

The assembly of vimentin depends on the concentration of $\mathrm{KCl}$ ions in the assembly solution (see Figure 4.14). The first value at time point $t=0$ shows the unassembled vimentin. The error bar for this value is higher than for the other points. After the start of the assembly the values for the diffusion coefficient decrease. After 20 min only a slight change is visible. The value at $60 \mathrm{~min}$ is lower for higher salt concentration.

We measured the assembly of vimentin at $22^{\circ} \mathrm{C}$ and $37^{\circ} \mathrm{C}$ with the 'reaction tube' 


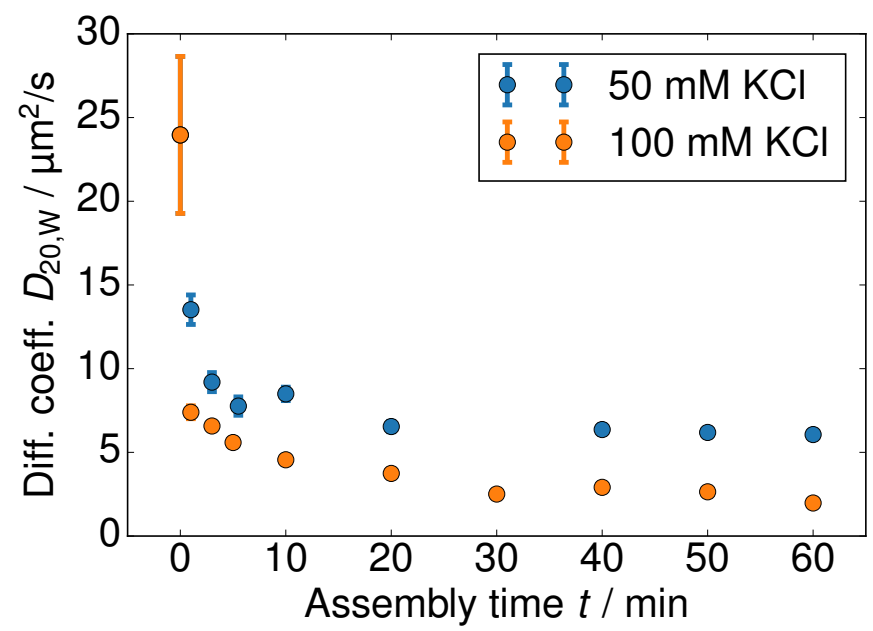

Figure 4.14: Salt dependence of vimentin assembly. Vimentin assembly experiments were carried out at different salt concentrations at a protein concentration of $50 \mathrm{mg} / 1$ and a temperature of $37^{\circ} \mathrm{C}$.

method (see Figure 4.15). For $22^{\circ} \mathrm{C}$ the diffusion coefficient decreases to $19 \mathrm{\mu m}^{2} / \mathrm{s}$ after one minute and further down to $10 \mu^{2} / \mathrm{s}$ after 20 minutes and stays around this value until the end of the measurement after 60 minutes. At $37^{\circ} \mathrm{C}$ the diffusion coefficient decreases to $6 \mathrm{\mu m}^{2} / \mathrm{s}$ already after one minute. The data points stem from single measurements. The two curves show a similiar behaviour but decline to different values after $60 \mathrm{~min}$. The early stages of the assembly show a similiar decrease. The temperature during the assembly plays a role for the diffusion of the particles. For a higher temperature the diffusion is faster and the assembly can take place at a higher rate, because particles 'meet' each other at a higher rate. At later times the assembly comes nearly to a hold. 
4 Results

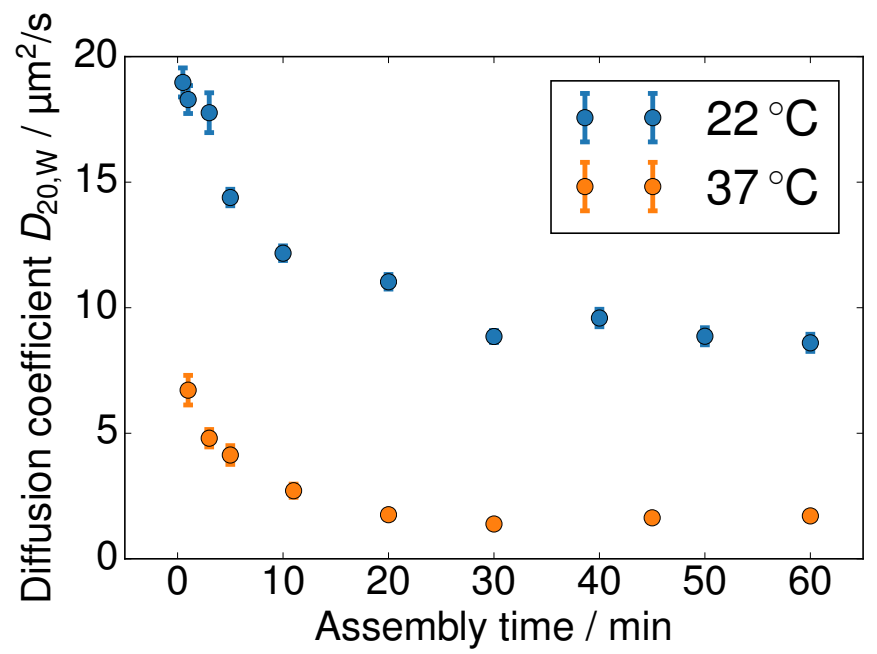

Figure 4.15: Temperature dependence of vimentin assembly. Vimentin assembly experiments were carried out at temperatures of $T=22^{\circ} \mathrm{C}$ and $T=37^{\circ} \mathrm{C}$ at $c_{\mathrm{Vim}}=200 \mathrm{mg} / \mathrm{l}$ and $c_{\mathrm{KCl}}=100 \mathrm{mM}$. The diffusion coefficient decreases faster at higher temperatures. 


\subsection{Microfluidic device for protein assembly}

For the experiments on intermediate filament assembly we employ a microfluidic mixing device that gives access to the relevant time scales of the processes. In the device, unassembled protein is brought into contact with ions that kickstart the assembly process. It is measured how the protein changes during the assembly. We employ a continuous flow device where a constant supply of protein and ions is maintained.

\subsubsection{Criteria for microfluidic mixing}

The purpose of the mixing device is to supply the ions into the center protein stream. The flow is laminar and therefore the mixing happens due to diffusion. We employ the concept of hydrodynamic focusing [31], where lateral streams are constraining a central stream. The central stream is narrowed down to a fraction of its original width, depending on the flow rates of the individual streams. Due to the smaller width, the diffusive spreading of the ions from the outer streams into the center takes places on a shorter timescale. The ions diffuse faster than the protein. Therefore the ions will diffuse into the protein jet while the protein is only diffusing out slowly.

In the first experiments, we use a mixing device with three inlets and a narrow channel after the mixing region [33, 34]. After the narrow part, the channel widens up and the

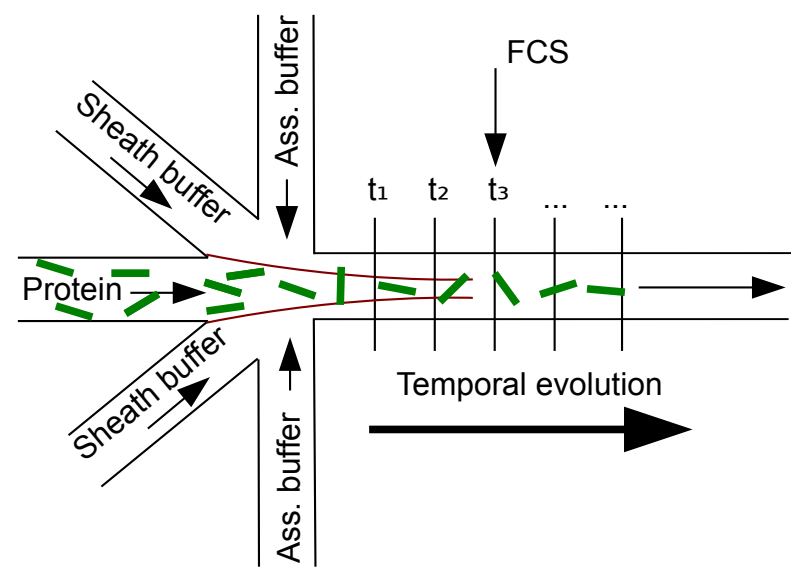

Figure 4.16: Five-inlet mixing device. The protein jet is focused by the lateral inlets. The diagonal inlets provide a layer of non-assembling buffer to pre-focus the protein stream before it comes into contact with the assembly buffer. FCS measurements are performed along the channel at several distances which correspond to specific time points in the studied process. 
flow rate is reduced. The narrow channel diameter promotes the spread of diffusive molecules over the whole channel width. For measuring with FCS in the wide outlet channel, the flow rate needs to be adjusted to this outlet channel.

Avoiding protein adsorption Proteins have the tendency to adsorb to surfaces [76]. This is important especially in microfluidic devices because of the high surface to volume ratio. Intermediate filament proteins adsorb to surfaces when the assembly is started. That makes it difficult to obtain results in microfluidic experiments. Therefore we try to prevent the adsorption of protein with the surface [77]. The blocking of the surface with PLL-g-PEG, BSA, Poloxamer 407, or lipids was tested for the assembly experiments, (see Chapter 3.3 .4 for the experimental details).

Mixing channel layout In order to avoid contact of vimentin with top and bottom surface we introduce a constriction in the central inlet channel. The protein stream is encapsulated by the assembly buffer stream. This prevents the protein from touching the walls in the direction of the plane of the inlets. For the perpendicular direction we introduce a step structure (see Section 2.3.3). Here, the protein inlet has a smaller height than the buffer inlets. The buffer solution flows around the protein stream also on the top and bottom side. The contact of protein to the device wall is thus minimized.

Since the central jet comes into contact with the assembly buffer already at the start of the hydrodynamic mixing, the variation in interaction times is large (see Figure 4.16). By introducing additional diagonal inlets, a barrier between the two reactants is created during the focusing process [35]. The barrier leads to a more instantaneous start of the interaction.

Channel cross-section Since we want to access the center of the channel with FCS, the cone of the focused laser beam should fit completely into the cross-section of the channel. Otherwise parts of the light cone are clipped off [33]. This would disturb the signal. For a given depth $d$, the diameter $D$ of a channel that holds the whole light cone is given by:

$$
D=\frac{2 A d}{\sqrt{n_{s}^{2}-A^{2}}}
$$


where $A$ is the numerical aperture of the objective and $n_{s}$ is the refractive index of the solution in the channel. For $A=1.2$ and $n_{s}=1.33$ :

$$
D=4.18 d
$$

For placing the detection volume in the center a channel height of $100 \mu \mathrm{m}$ is chosen at a channel diameter of $200 \mu \mathrm{m}$ (see Figure 4.17). Another aspect that needs to be considered for the layout of the channel cross-section is the working distance of the objective. The working distance of the used objective Olympus UPLSAPO 60XW is $280 \mu \mathrm{m}$. The glass slides (No. 1) that are used for the microfluidic devices have a thickness of $150 \mu \mathrm{m}$. The remaining distance is $130 \mu \mathrm{m}$. To be able to scan the whole channel the height should not exceed this value. Taking into account these aspects, we set the height of the channel to $100 \mu \mathrm{m}$ at a width of $200 \mu \mathrm{m}$.

Flow velocity Both diffusion and active flow govern the shape of the ACF curve in an FCS experiment (see Section 2.4.1). When the flow velocity is too high the influence of the diffusion on the measured ACF curve becomes small and the shape of the curve is mainly governed by the active flow. This makes it hard to determine the characteristics of the diffusion. The flow speed of the protein solution in the device should be limited in a way that the FCS measurement still yields reliable values for the diffusion coefficient.

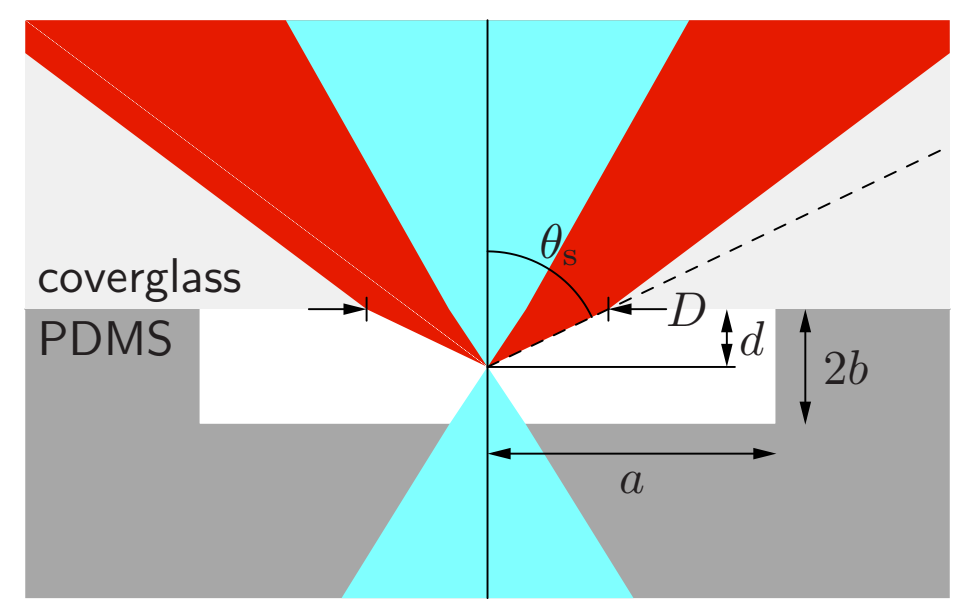

Figure 4.17: Focus cone in microfluidic channel. $D$ - Diameter of the light cone, $d$ - Depth of the focus, $2 b$ - height of the channel, $2 a$ - width of the channel. Figure taken with permission from 33 . 


\section{Results}

The influence of the diffusion and the active flow on the curve can be compared by determining the diffusion time $\tau_{\text {diff }}$ and the flow time $\tau_{\text {flow }}$. The diffusion time is the characteristic time a molecule spends in the detection volume when it is only moved by diffusion. The flow time is the time a molecule spends in the detection volume when it is only translated by active flow. The diffusion time $\tau_{\text {diff }}$ depends on the diffusion coefficient $D$ and the radius of the detection volume $w_{\mathrm{xy}}$ (see Section 2.4.1).

$$
\tau_{\text {diff }}=\frac{w_{\mathrm{xy}}^{2}}{4 D}
$$

The flow time depends on the flow velocity $v$ and the radius of the detection volume $w_{\mathrm{xy}}$ 60]:

$$
\tau_{\text {flow }}=\frac{2 w_{\mathrm{xy}}}{v}
$$

In order to be able to detect diffusion in a flowing stream, the diffusion time should be at most of the same magnitude as the flow time:

$$
\frac{\tau_{\text {diff }}}{\tau_{\text {flow }}}=\frac{w_{\mathrm{xy}} v}{8 D}=1
$$

For vimentin tetramers with $D \approx 25 \mu \mathrm{m}^{2} / \mathrm{s}$ and a detection volume of $w_{\mathrm{xy}}=260 \mathrm{~nm}$, we calculate a flow velocity of $v=570 \mu \mathrm{m} / \mathrm{s}$. For smaller diffusion coefficients that occur during assembly of vimentin the optimal flow velocity is even lower (see Table 4.1). The ratio of $\tau_{\text {diff }}$ to $\tau_{\text {flow }}$ depends on radius of the detection volume.

Figure 4.18 a shows calculated autocorrelation curves for one diffusion coefficient at varying flow velocities. The flow changes the shape of the curves and shifts the curve to smaller lag times. The graph in Figure 4.18b shows calculated curves for diffusion coefficients of $D=23 \mu^{2} / \mathrm{s}$ and $D=0 \mathrm{\mu m}^{2} / \mathrm{s}$ at a flow speed of $v=0.5 \mathrm{~mm} / \mathrm{s}$. These two curves are the boundaries for the measurement of vimentin assembly. All diffusion coefficients that occur during the measurement are expected to lie in this range. At this flow velocity, the impact of the diffusion coefficient on the shape of the ACF is small, but still detectable. This shows the need for low flow speeds when measuring diffusion properties.

The Péclet number for the vimentin tetramers with a diffusion coefficient of $D=$ $25 \mathrm{\mu m}^{2} / \mathrm{s}$ in a channel of $200 \mu \mathrm{m}$ width at a flow velocity of $v=1 \mathrm{~mm} / \mathrm{s}$ is of the order 


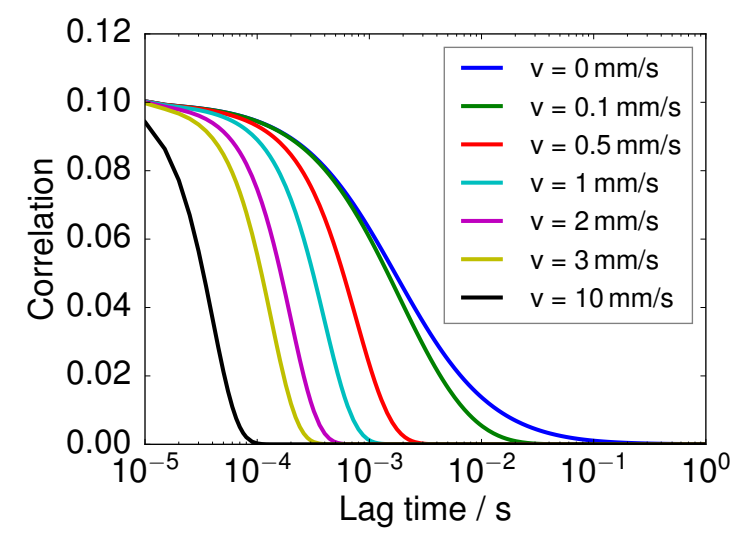

(a)

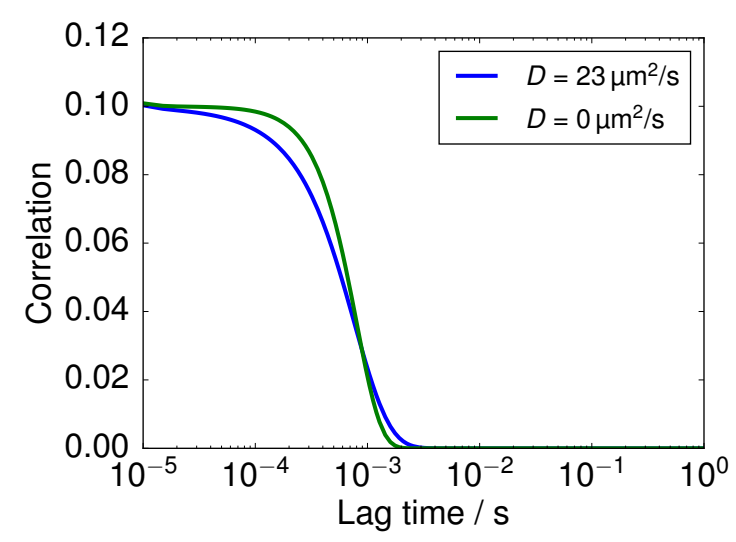

(b)

Figure 4.18: Influence of flow on the auto-correlation curves (a) Variation of the flow speed at a constant diffusion coefficient with $D=23 \mu \mathrm{m}^{2} / \mathrm{s}$. (b) Influence of a changing diffusion coefficient at a constant flow of $v=0.5 \mathrm{~mm} / \mathrm{s}$.

Table 4.1: Estimation of the flow speed for FCS measurements. For a given detection volume radius $w_{\mathrm{xy}}$ and a diffusion coefficient $D$ we estimate the flow speed so that $\tau_{\text {diff }} \approx \tau_{\text {flow }}$.

\begin{tabular}{c|c|c}
\hline \hline$w_{\mathrm{xy}} / \mathrm{nm}$ & $D / \mu \mathrm{m}^{2} / \mathrm{s}$ & $v / \mu \mathrm{m} / \mathrm{s}$ \\
\hline 260 & 25 & 770 \\
260 & 10 & 307 \\
260 & 5 & 150 \\
260 & 1 & 30 \\
\hline \hline
\end{tabular}




\section{Results}

of $10^{4}$. For the salt ions $\left(D=1800 \mathrm{\mu m}^{2} / \mathrm{s}\right)$ that trigger the assembly the Péclet number is of the order of 100 .

\subsubsection{Simulations of microfluidic flow}

With numerical simulations we can test parameters for the mixing device before producing the actual device. The goal of the simulations is to find the optimal parameters for the mixing device. We employ a five-inlet device as introduced in Section 4.4.1. The numerical simulations are performed in COMSOL. The x-axis is defined in parallel to the center of the protein inlet, while the y-axis is defined parallel to the side buffer inlet. The $z$-direction is defined perpendicular to that. The protein enters via the central inlet and is enclosed by two streams containing only buffer solution. The two outer streams provide the ions that trigger the assembly. The two inner streams form a layer between the assembly buffer and the protein. The ions in the assembly buffer diffuse into the protein jet. By introducing the buffer layer in between the protein jet and the assembly buffer, the distance that the ions need to diffuse is minimized.

The aim of introducing the step structure is to avoid the contact between the assembling protein and the surface of the device [20]. The outer buffer streams encapsulate the inner protein jet in order to shield it from the surface. The simulations show that the protein is spreading towards the top of the device after leaving the outlet channel, but the concentration is not reaching the maximum (see Figure 4.20). The vimentin concentration is decreasing fastly towards the later parts of the channel. This behavior is also visible in Figure 4.20. The vimentin concentration is decreasing during the first hundred $\mu \mathrm{m}$ after the mixing region to a quarter of the initial value. The protein jet has an expected width of $20 \mu \mathrm{m}$ taking into account the ratios of the flow rates. Starting with this width, the estimated diffusion time for the protein jet to double in width is less than one second $\left(\tau \sim w^{2} / D\right)$. This is also visible in the simulation were the protein concentration drops fast after the mixing region. In the simulation, the concentration drops from $200 \mathrm{mg} / 1$ to $50 \mathrm{mg} / \mathrm{l}$ after a distance of around $200 \mu \mathrm{m}$. At a flow velocity of around $10 \mathrm{~mm} / \mathrm{s}$, the protein travels for 0.02 seconds for these $200 \mu \mathrm{m}$. This jet is spreading fast due to diffusion of the vimentin molecules towards the side streams. The decrease in concentration seen in the simulation is faster than we would expect it from the estimation above.

In Figure 4.21 the protein and salt concentrations along the central line of the channel 
are depicted. The protein concentration shows a decrease from the intersection of the mixing, while the salt concentration is increasing rapidly in the center of the stream.

The expected time for the salt to diffuse into the center of the protein jet is on the order of $10 \mathrm{~ms}$ due to the high diffusivity of the salt ions compared to vimentin. In the simulation, the salt concentration in the center of the channel increases also at a high rate (Figure 4.21). Here, the salt concentration increases to the concentration of $70 \mathrm{mM}$ during the first few hundred $\mu \mathrm{m}$ after the first contact of the salt and the central stream.

For investigating the effect of the constraining step on the flow profile, we compare simulations with and without the step structure. We used an inflow of fluorescein into the central channel. Figure 4.19 shows that the central jet is constrained by the lateral streams. The flow velocity is the same in both simulations, but due to the different inlet heights, the flow rates are different. For the inlet with the full height, the flow rate is higher. This causes the the fluorescein stream to extend further into the mixing region. The flow speed that is used for this simulation is not high enough to prevent the contact of the protein to the wall. At higher flow speeds the effect becomes more prominent. 


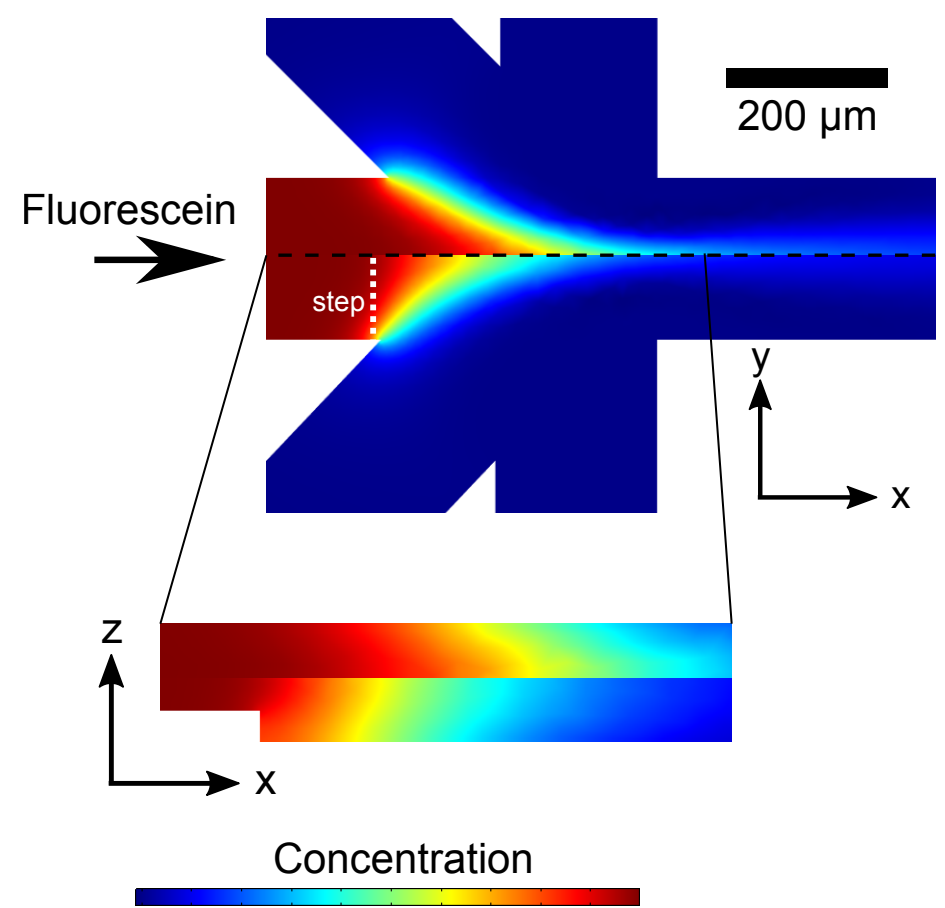

Figure 4.19: Comparison of the simulations for devices with and without the constraining step. The top parts of the figures show the device without step and the lower parts shows the device with step. The step is indicated in the lower part. The flow rates of the simulation are $100 \mu \mathrm{l} / \mathrm{hr}$ in the side inlet, and $5 \mu \mathrm{l} / \mathrm{hr}$ in the diagonal and the central fluorescein inlet. 
a )
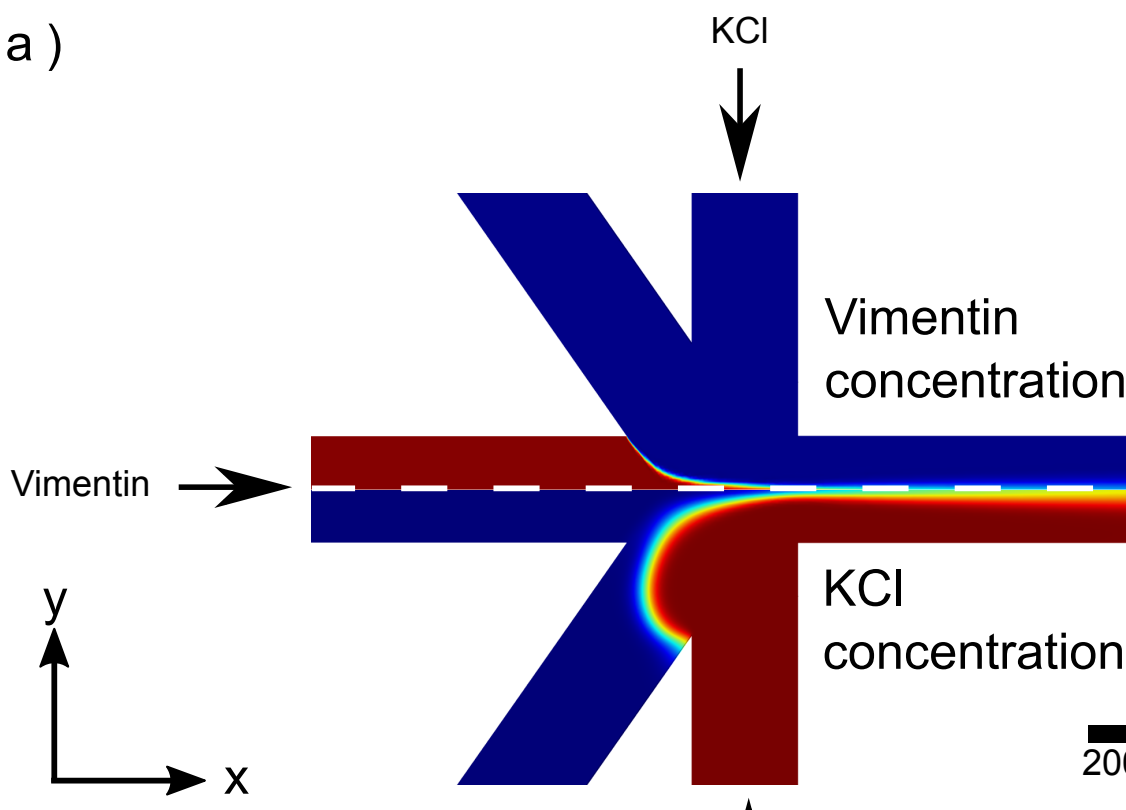

b)

Vimentin concentration
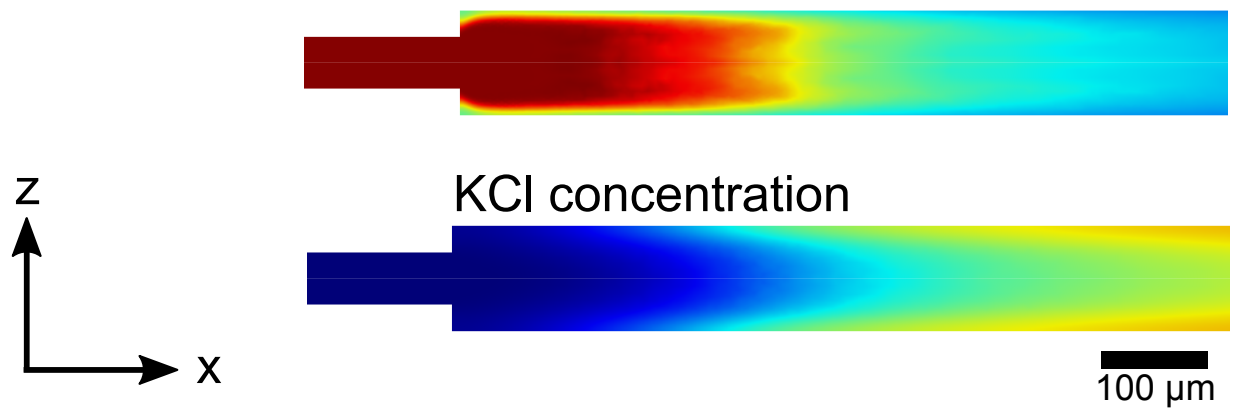

Figure 4.20: Results of the vimentin flow simulation. The figure shows composites of the vimentin concentration (upper part) and the $\mathrm{KCl}$ concentration (lower part) of the flow simulation in a five-inlet device with a constraining step. The average flow speed in the protein inlet channel is $490 \mu \mathrm{m} / \mathrm{s}$ $(35 \mu \mathrm{l} / \mathrm{h})$, the average flow speed of the diagonal inlet is $210 \mu \mathrm{m} / \mathrm{s}(15 \mu \mathrm{l} / \mathrm{h})$, and the average flow speed of the side inlet channel is $2100 \mu \mathrm{m} / \mathrm{s}(150 \mathrm{\mu l} / \mathrm{h})$. (a) Top view of the device. (b) Perpendicular view of the cut-plane along the dashed line in Figure a). The influence of the constriction in the vimentin inlet is shown here. 

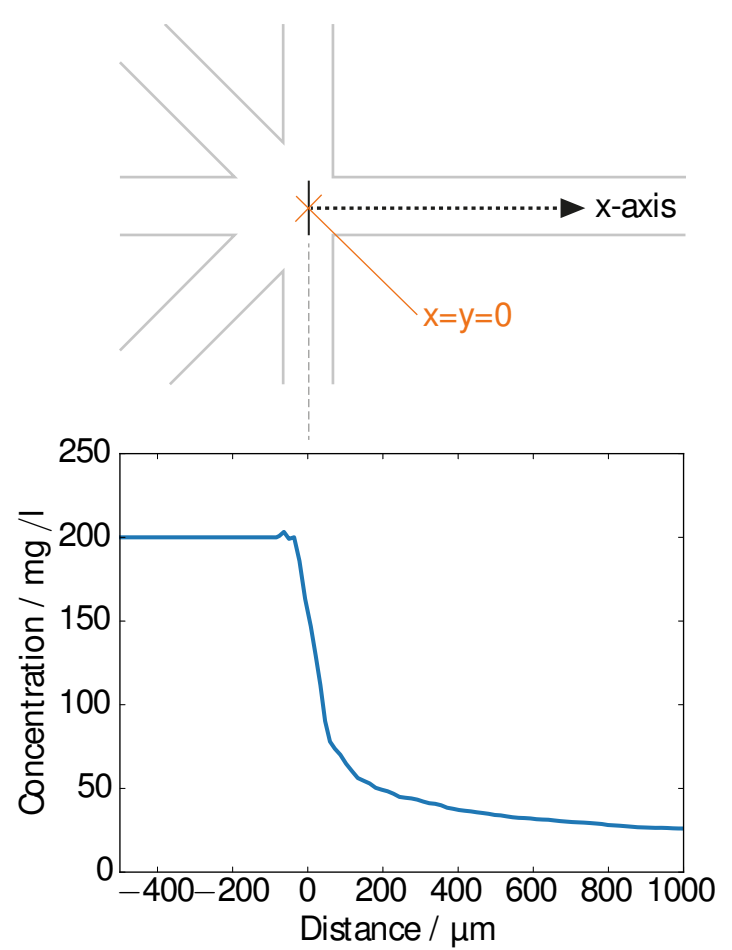

(a)

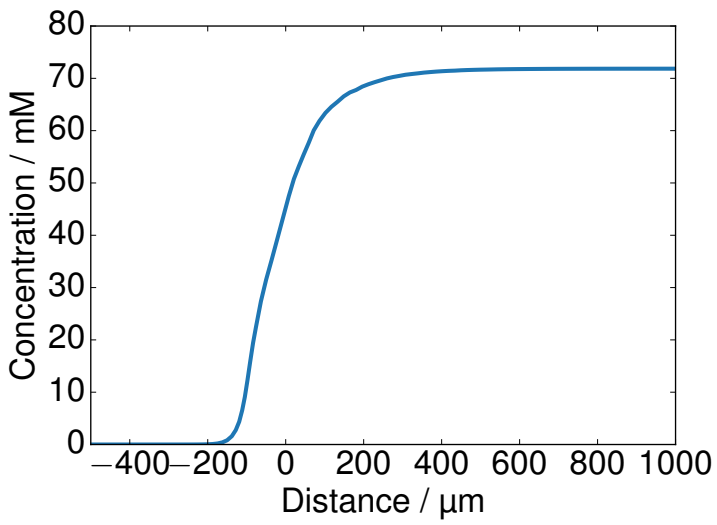

(b)

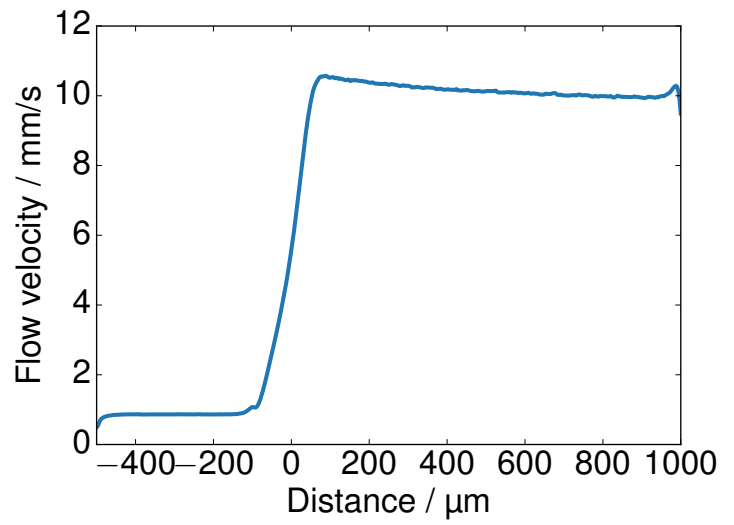

(c)

Figure 4.21: Simulation of protein and salt concentration along the channel. The graph shows the (a) vimentin concentration and the (b) salt concentration along the center of the device. The intersection of all inlet channels is situated at $0 \mu \mathrm{m}$. The flow conditions are the same as in Figure 4.20. (c) The flow velocity along the channel. The peak at the end of the simulated channel and the decrease in flow velocity at a constant channel width (from $600 \mu \mathrm{m})$ are numerical artifacts. 


\subsubsection{Fabrication of the final mixing device}

For the production of the mixing device a method called micro-molding in capillaries (MIMIC) is employed (see Section 3.3.2). A brightfield microscopy image is shown in Figure 4.22. The constraining structure at the top and at the bottom side of the channel are visible in the two images. A comparison between the simulation and a experimental flow device is shown in Figure 4.23. The device is tested with fluorescein. The simulation shows a decrease in the fluorescein concentration at the top of the device. In the experiment, the fluorescein spreads equally in the channel. The step structure does not lead to a lower fluorescein concentration at the bottom of the device. The contact between the lower surface of the device and the fluorescein is not prevented. To prevent the contact of the fluorescein and the surface, the flow speeds from the side inlets need to be higher. With higher flow rates, the fluorescein has less time to diffuse towards the channel walls and stays more confined in the center of the jet. Otherwise the fluorescein diffuses out of the central channel too fast and spreads into the whole volume. The step structure in the protein inlet has a width of $200 \mu \mathrm{m}$ at a height of $50 \mu \mathrm{m}$. The effect of the encapsulation in the z-direction is reduced by the extended rectangular shape of the constriction in $\mathrm{x}$-direction. The lateral fluid jets are not reaching into the area above and below the middle of the central inlet.

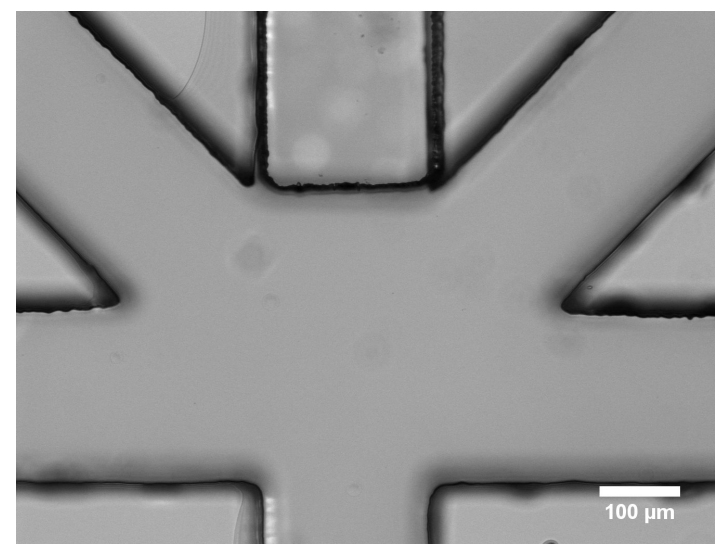

(a)

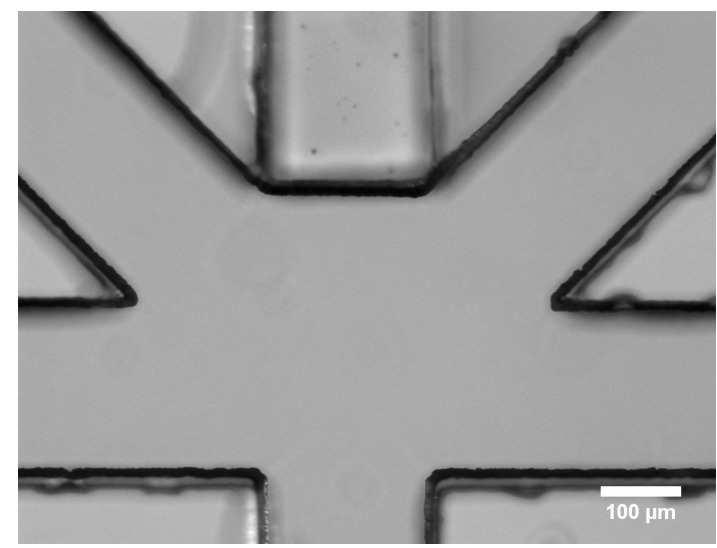

(b)

Figure 4.22: Five-inlet mixing device with constraining step. Bright-field image of the five-inlet mixing device with constraining step. (a) Focused on the constraining NOA step structure on the bottom of the channel. (b) Focused on the constraining step structure at the top of the channel in the PDMS layer. 
4 Results

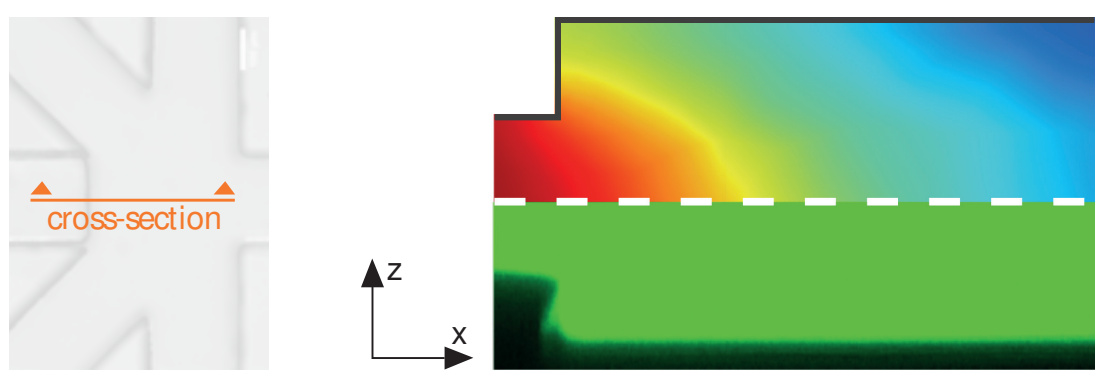

Figure 4.23: Comparison of simulation and experiment for step structure. The image shows the simulated (upper part) and measured fluorescein concentration (lower) in the mixing area. 


\subsection{Vimentin assembly in microfluidic flow}

In this section we will present the results of the vimentin assembly experiments in microfluidic flow. The assembly of vimentin is a process over a broad range of timescales. The formation of ULFs happens in seconds, while the formation of longer filaments takes minutes to hours. To access the longer time scales, we employ the bulk methods described in Section 4.3. The bulk approach is limited in the time resolution. For the shorter time scales, we use a microfluidic mixing device. Here, the mixing does not require manual interaction. The fluids are mixed in a continuous manner in the device. Thus, the earliest accessible time points are only limited by the geometry of the device.

At the beginning of the experiment we take an overview image by scanning the sample with a short acquisition time (see Figure 4.24). The image shows the count rate at every point. From this image we determine the exact sample position for the following scans. For the FCS measurements, we acquire data at every sample point for 3 times 20 seconds. The acquisition time per scan point is a trade-off between the total duration of the scan and the amount of data per point.

\subsubsection{Unassembled vimentin in flow}

We first study the flow of vimentin in the microfluidic mixing device without ions. We let vimentin flow through the five-inlet device with a constriction in the center channel. A typical measurement is shown in Figure 4.25. The vimentin concentration is $c_{\text {Vim }}=200 \mathrm{mg} / \mathrm{l}$. The mean flow speed and the flow rate in the protein inlet are $49 \mu \mathrm{m} / \mathrm{s}$ and $3.5 \mu \mathrm{l} / \mathrm{h}$, in the diagonal inlets $20 \mu \mathrm{m} / \mathrm{s}$ and $1.5 \mu \mathrm{l} / \mathrm{h}$ and in the side inlets $208 \mu \mathrm{m} / \mathrm{s}$ and $15 \mu \mathrm{l} / \mathrm{h}$. The total mean flow velocity is $505 \mu \mathrm{m} / \mathrm{s}$ and the total flow rate is $36.5 \mathrm{ll} / \mathrm{h}$. A line scan across the channel shows the dependency of the measurements on the distance to the center of the protein stream (see Figure 4.25). The intensity shows the position of the vimentin stream in the channel (Fig $4.25 \mathrm{a}$ ). The stream was not fully aligned to the scan range and appears shifted to the left. In the plots the center of the protein stream is at $y=12 \mu \mathrm{m}$. The decrease of the flow velocity towards the outer parts is expected since the flow follows a parabolic flow profile (Fig. 4.25). For the outer parts of the channel the fits yield smaller values for the flow velocity while the diffusion coefficient is increasing. The diffusion coefficient increases towards the edges of the channel. The flow velocity and the diffusion coefficient are free parameters in the 


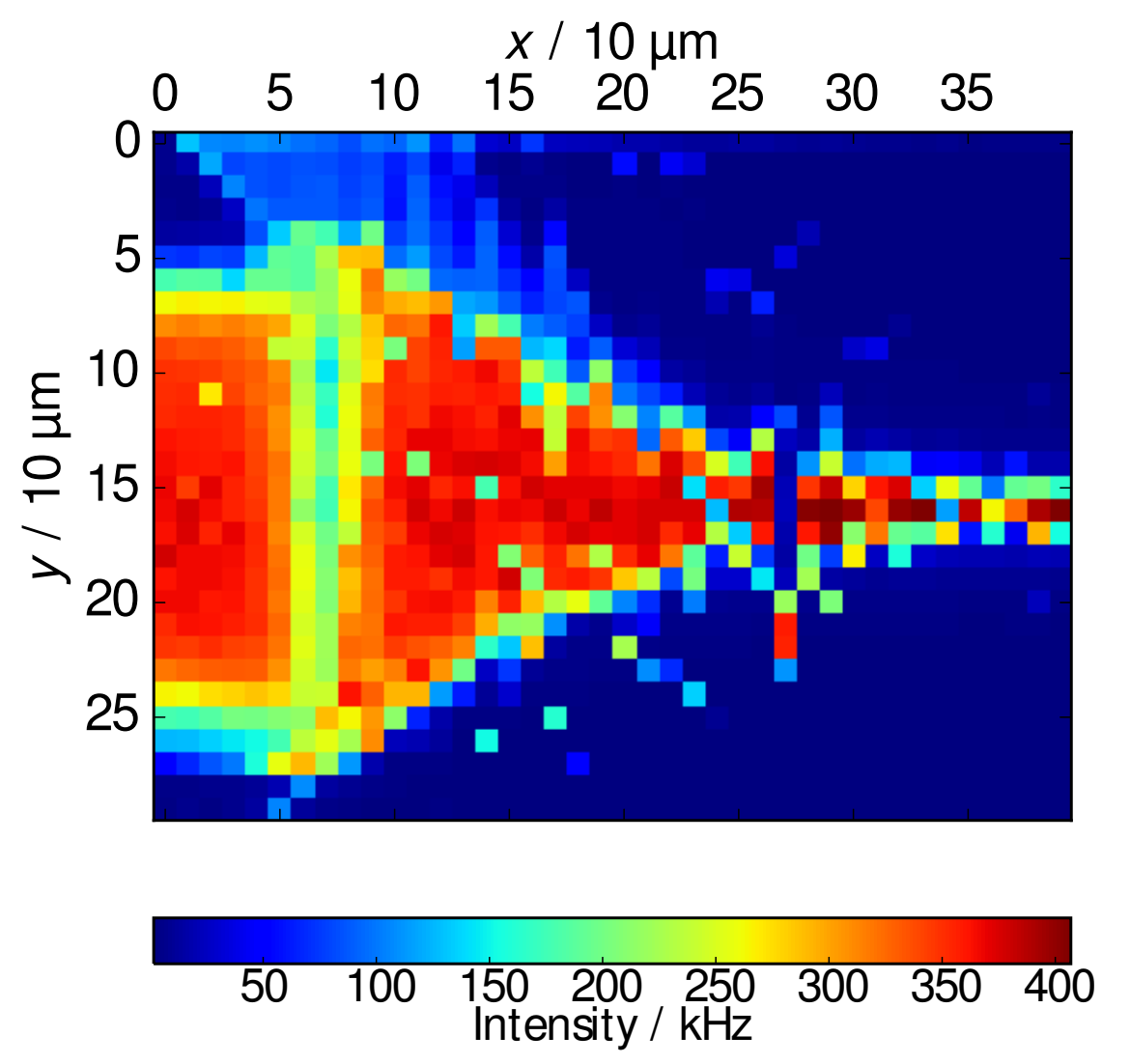

Figure 4.24: Scan of the mixing area. The image shows a count rate map of the mixing area. The acquisition time per pixel is $1 \mathrm{~s}$. The step size is $10 \mu \mathrm{m}$. The fluorescently labeled protein comes in from the left side and is hydrodynamically focused by the lateral streams. The region at the exit of the central inlet channel to the mixing area appears less intense due to the constriction in the central inlet. The line at $x=25 \mu \mathrm{m}$ is shifted due to a temporal shift of the flow conditions. The flow conditions are the same as in Figure 4.25.

fit. The fit can determine the diffusion coefficient at the edges of the stream only to a small extent. This leads to unrealistically high values towards the outer part of the stream. Smaller molecules in the stream that are diffusing faster to the outer parts of the stream could cause an increase of the diffusion coefficient.

For more reliable values for the diffusion coefficient it might be advantageous to use a fixed flow velocity in the fit. An estimate for the flow profile in the experiment can be obtained from simulations with the same parameters. The values from the simulation can then be used in the fitting process. The increasing diffusion coefficient is inversely 
following the decreasing intensity (Fig. $4.25 \mathrm{~b}$ ). The values for the count rate per molecule are also decreasing towards the outer area of the stream (Fig. 4.25d). 


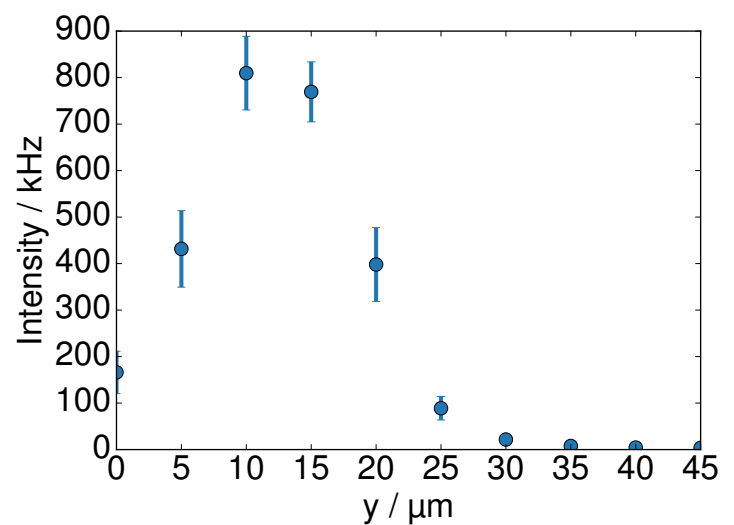

(a)

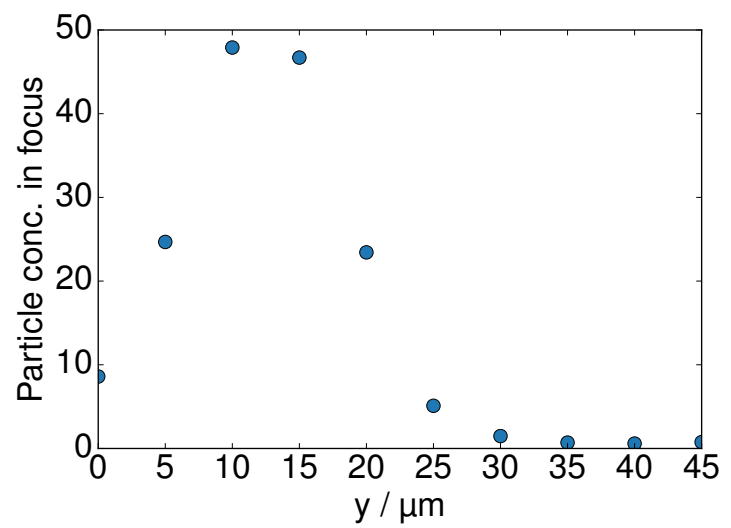

(c)

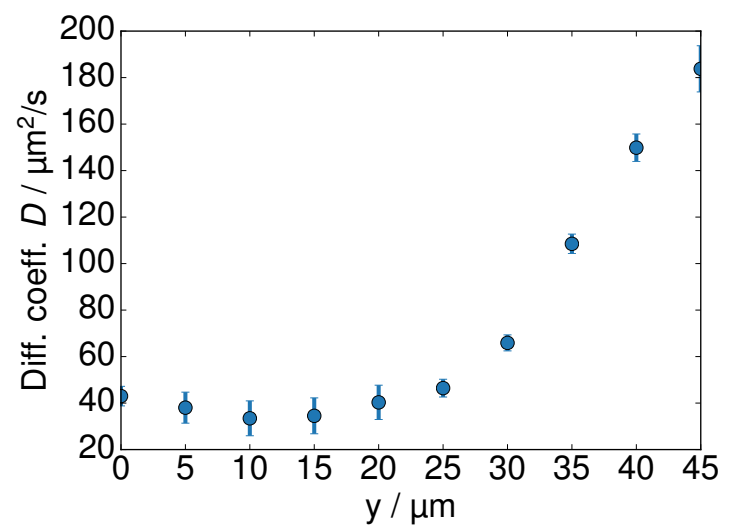

(b)

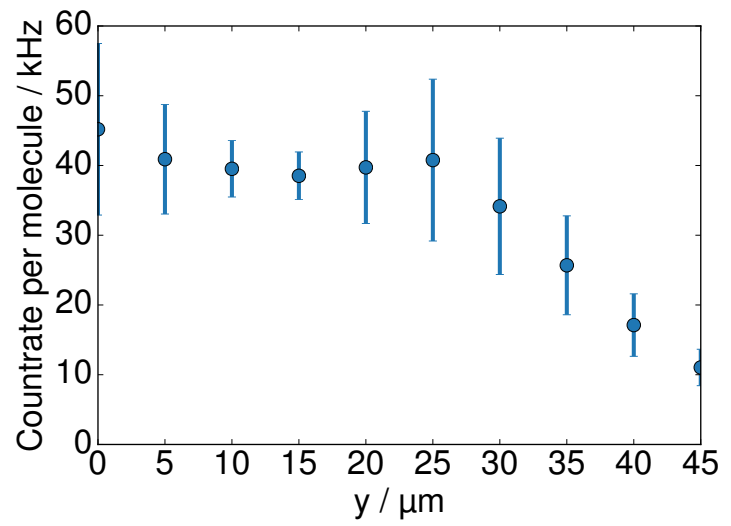

(d)

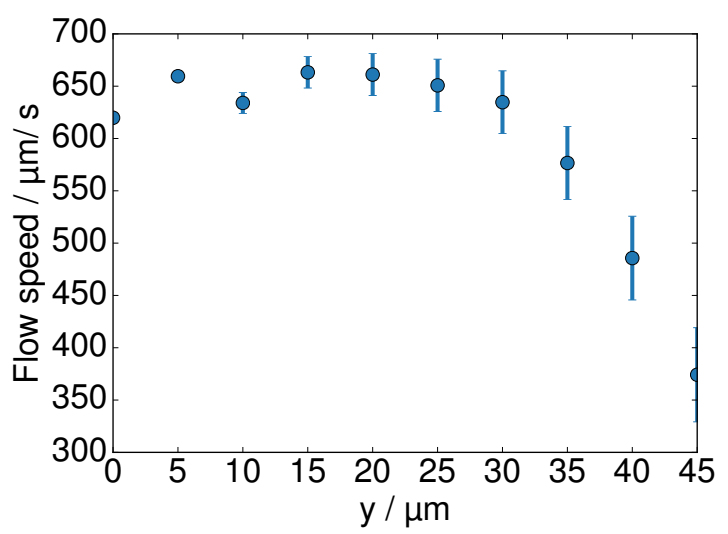

(e)

Figure 4.25: FCS scan of vimentin stream without assembly trigger at $\mathbf{2} \mathbf{~ m m}$ downstream. The protein concentration is $200 \mathrm{mg} / \mathrm{l}$. The mean flow speed in the protein inlet is $49 \mu \mathrm{m} / \mathrm{s}(3.5 \mu \mathrm{l} / \mathrm{h})$, in the diagonal inlets $20 \mu \mathrm{m} / \mathrm{s}$ $(1.5 \mu \mathrm{l} / \mathrm{h})$ and in the side inlets $208 \mu \mathrm{m} / \mathrm{s}(15 \mu \mathrm{l} / \mathrm{h})$. Measurement across the channel at a position $2 \mathrm{~mm}$ downstream. (a) Intensity. (b) Diffusion coefficient. (c) Particle concentration in focus. (d) Count rate per molecule. (e) Flow velocity. 


\subsubsection{Vimentin assembly in flow}

For studying the early assembly stages of vimentin, we mix protein with buffer containing ions to trigger the assembly in the device.

The mean flow speed in the protein inlet is $49 \mu \mathrm{m} / \mathrm{s}(3.5 \mu \mathrm{l} / \mathrm{h})$, in the diagonal inlets $20 \mu \mathrm{m} / \mathrm{s}(1.5 \mu \mathrm{l} / \mathrm{h})$ and in the side inlets $208 \mu \mathrm{m} / \mathrm{s}(15 \mu \mathrm{l} / \mathrm{h})$. At a total mean flow velocity of $505 \mathrm{\mu m} / \mathrm{s}$ the protein travels along the channel for 10 seconds until it reaches the probed area at $x=5 \mathrm{~mm}$. The vimentin concentration is $c_{\text {Vim }}=400 \mathrm{mg} / \mathrm{l}$ and the potassium chloride concentration is $c_{\mathrm{KCl}}=200 \mathrm{mM}$.

Figure 4.26 shows linescans across the channel at $5 \mathrm{~mm}$ and $10 \mathrm{~mm}$ downstream from the intersection of the inlet channels. The intensity distribution shows a symmetric profile (Fig. 4.26a). The intensity at position $x=10 \mathrm{~mm}$ has a lower maximum than for the plot at $x=5 \mathrm{~mm}$. The values at the edges of the intensity profile are higher for $x=10 \mathrm{~mm}$ and the complete intensity profile is shallower. This can be explained by the diffusion towards the sides of the channel. Also adsorption of protein to the channel walls might decrease the intensity of the protein stream. The measurement of the diffusion coefficient shows a similar profile for both positions (Fig. 4.26 b). The plots of the particle concentration show a similar behavior as the intensity curves with a lower maximum for the curve at $x=10 \mathrm{~mm}$ (Fig. 4.26 ). Since the profile for the count rate per molecule stays the same for both positions, the difference in the intensity can be explained by the change in particle concentration. The count rate per molecule has a similar maximum value, but decreases less to the sides for $x=10 \mathrm{~mm}$ (Fig. 4.26 d). The flow speed profiles are similar for both positions (Fig. 4.26e). An decrease of the diffusion coefficient along the channel is not detected. The methods used might not be sensitive enough or the assembly reaction was not started in the stream. 


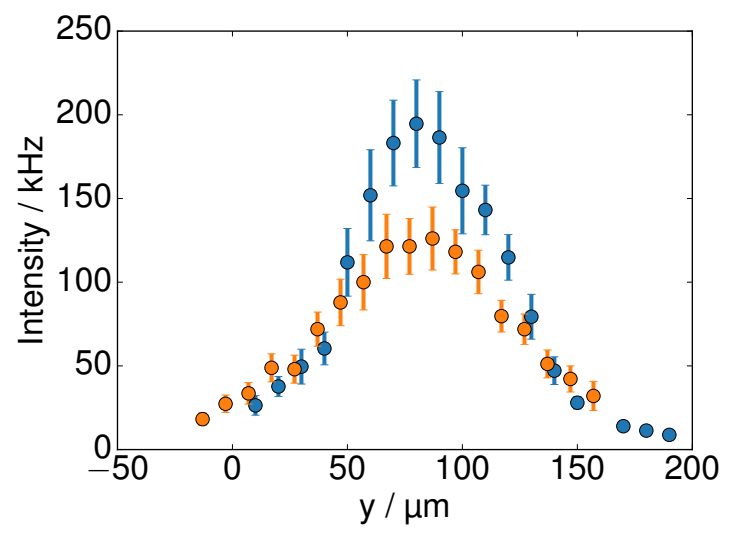

(a)

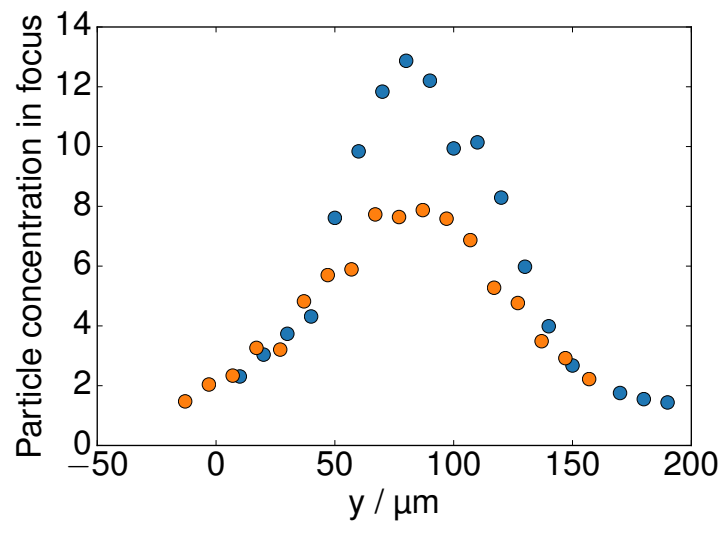

(c)

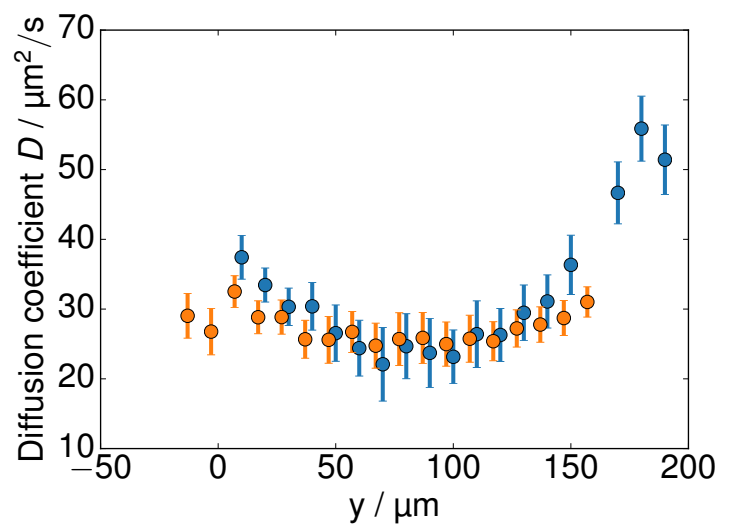

(b)

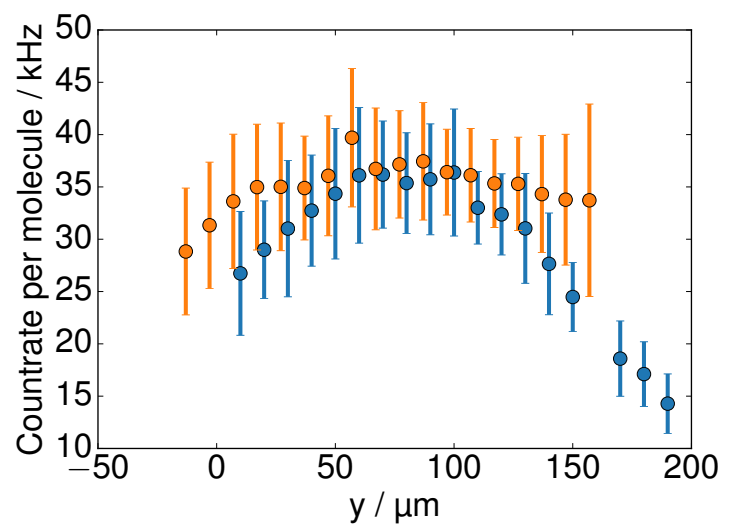

(d)

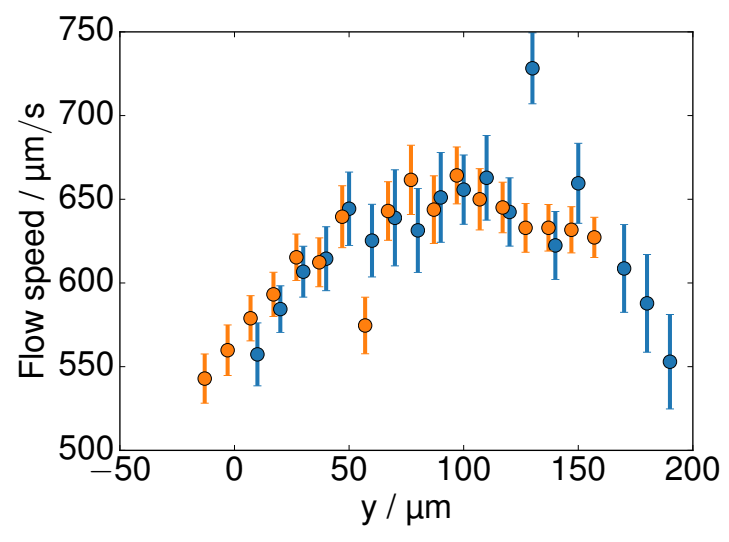

(e)

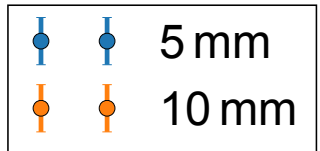

(f)

Figure 4.26: Comparison of vimentin at $\mathbf{5} \mathbf{~ m m}$ and $\mathbf{1 0} \mathbf{~ m m ~ d o w n s t r e a m . ~ T h e ~}$ mean flow speed in the protein inlet is $49 \mu \mathrm{m} / \mathrm{s}(3.5 \mu \mathrm{l} / \mathrm{h})$, in the diagonal inlets $20 \mu \mathrm{m} / \mathrm{s}(1.5 \mu \mathrm{l} / \mathrm{h})$ and in the side inlets $208 \mu \mathrm{m} / \mathrm{s}(15 \mu \mathrm{l} / \mathrm{h})$. (a) Intensity. (b) Diffusion coefficient. (c) Particle concentration in focus. (d) Count rate per molecule. (e) Flow velocity. (f) Legend. 


\section{Discussion}

After presenting the results of this thesis in the previous chapter we will now discuss the results.

\subsection{FCS setup}

The FCS setup that was build during this thesis project is presented in Chapter 4.1. Here, we discuss the performance of the instrument. The setup is used for the study of vimentin assembly in bulk and in microfluidic mixing. The calibrated size of the detection volume is $260 \mathrm{~nm}$.

We can measure the mobility of molecules in static solutions and in flow. In flow experiments the analysis is more complicated since the active flow is shaping the ACF together with the diffusion. The flow velocity needs to be fitted at the same time as the diffusion coefficient. Our FCS setup employs one laser focus. Besides one-focus FCS also dual-focus FCCS can be used to measure molecules in flow [55, 60. In dual-focus FCCS the flow velocity can also be derived from the CCF between the two foci. Having the flow velocity determined separately, the fit for the diffusive motion is more reliable. However, the hardware setup for dual-focus FCCS is more complex than for one-focus FCS.

\subsection{Vimentin labeling}

For studying the assembly of vimentin with FCS, the fluorescent labeling is a prerequisite. The results of the vimentin labeling are shown in Section 4.2. We investigate how we can remove the free dye component in the vimentin solution. We employ several methods to accomplish a low content of free dye molecules in the vimentin solution. Long dialysis times could not remove the free dye component completely. The dialysis 


\section{Discussion}

takes place at a higher protein concentration than the actual experiments. At higher concentrations the free dye might be bound unspecifically to the vimentin tetramers. When the unbound dye has a concentration-dependent binding affinity, it might be released upon dilution. Longer size exclusion chromatography helps in removing the free dye. Although free dye molecules can not be removed completely, the vimentin solution can be used in the assembly experiments. For analyzing FCS curves, we use a two-component fit.

Also other studies have shown that removing free fluorescent dye after labeling is not always possible and a second component is necessary for a fitting. Nath et al. labeled $\alpha$-synuclein with Alexa 488 C5 maleimide and found a free dye component of $20 \%$ [78]. In the study of Krouglova et al. tubulin was labeled with TAMRA and a free dye component was found after purification as well [79].

\subsection{Vimentin assembly in bulk}

The results of the vimentin assembly experiments are described in Section 4.3. We investigated the influence of external parameters on the assembly comparing the results of the measurements with the reaction tube method and the continuous method. The experiments with the same parameters show similar results. We saw a dependence on the salt concentration, the protein concentration and the temperature.

Standard FCS analysis can only determine single diffusion coefficients, thus averaging over a distribution of particles with varying diffusion properties. An alternative is the maximum-entropy data evaluation method (MaxEnt), which allows for estimating the distribution of diffusion coefficients from FCS data 80, 81]. This approach can be advantageous for the analysis of FCS data of intermediate filament assembly, since the assembly process shows a wide distribution of concurrent particle sizes. Since the fitting process is very sensitive due to the large numbers of fit parameters, we did not employ this method. Apart from FCS in combination with MaxEnt, distribution of intermediate filament assembly states can be studied with electron microscopy [23]. EM provides only static data, while FCS yields data in solution.

Other methods for studying the assembly are atomic force microscopy (AFM) [21], electron microscopy (EM) [82, fluorescence microscopy [17] and dynamic light scattering [22]. Another study used monte-carlo simulations for investigating the assembly [83]. 


\subsection{Microfluidic device for protein assembly}

The microfluidic mixer that we use for studying the assembly of vimentin is described in Section 4.4. The aim was to build a mixing device that can be used to study vimentin assembly with FCS. We employ a five-inlet device with a constraining step for the central inlet. The two additional diagonal channel pre-focus the central stream, so that the interaction with the outer streams takes place at a defined time.

We employed simulations to study the spread of vimentin in the inlet channel. One criterion for the microfluidic mixing device was the compatibility with the FCS measurements. For this reason, the flow velocity needs to be adjusted so that the impact of the diffusion on the ACF is still detectable.

We introduced a constriction of the central inlet to avoid the protein from adsorbing to the walls. That works well for higher flow rates. The protein concentration at the upper and lower surfaces of the channel is lower. However, at the low flow rates necessary to perform the FCS measurements the protein concentration is reduced, but only by a small amount.

\subsection{Vimentin assembly in microfluidic flow}

The results the vimentin assembly in micro-flow at $5 \mathrm{~mm}$ and $10 \mathrm{~mm}$ in Section 4.5 do not show a change in the diffusion coefficient. Here, we discuss possible reasons. On the one hand, the salt concentration at the center of the stream might not be high enough to start the assembly. In that case only unassembled molecules are detected. The simulations in Section 4.4 .2 show that the salt is diffused into the center of the channel already after $300 \mathrm{\mu m}$. Another explanation would be, that the assembly is started, but the assembling vimentin molecules adsorb to the wall while only the unassembled molecules stay in the stream. In this case, we would also detect only unassembled molecules. Since assembling molecules become larger and thus less diffusive, they diffuse out of the channel center at a lower rate. This makes it unlikely that they are not detected by the FCS.

The assembly of intermediate filaments has also been studied in micro-flow with SAXS [20]. 
5 Discussion 


\section{Conclusions and Outlook}

The goal of this thesis was to build a setup suitable for fluorescence correlation spectroscopy and to investigate the assembly of intermediate filaments in bulk and in microfluidic channels. The FCS setup was constructed and then calibrated by measuring the diffusion of molecules with a well-known diffusion coefficient.

We performed measurements of the vimentin assembly in bulk. We confirmed the dependence of the assembly on the protein concentration, salt concentration and temperature.

For accessing the early time scales of the assembly we built a microfluidic mixing device. Another reason to use this device was to overcome the low temporal resolution of FCS caused by the measuring time of several 10s of seconds. The microfluidic flow device maps the temporal evolution to a spatial axis. We assessed the properties of the device with numeric simulations. We tested the device and used it for the experiments on intermediate filament assembly. We encountered problems with the adsorption of proteins to the channel walls. The use of the following methods did not prevent the adsorption: The surface treatment with PLL-g-PEG, BSA or Poloxamer 407. The use of lipid coatings seems to be promising for the blocking of the surface. We employed a microfluidic device with five inlets containing a constriction in the central inlet channel. This led to a better prevention of the adsorption. The combination of the constriction device and the lipid coatings might be a promising approach to fully solve the problem of the protein adsorption.

Outlook The confocal volume of the FCS setup can be calibrated in several ways. Another method to calibrate the confocal volume is the measurement of subresolution fluorescent beads [84. The high translational resolution which is necessary for the bead scanning is achieved with a piezo positioning system.

With FCS we detect the diffusion properties of molecules and can derive diffusion coefficients and concentrations. With two-color FCCS it is possible to measure the 
6 Conclusions and Outlook

binding of molecules which are labeled differently. For example, the co-assembly of two differently labeled intermediate filament types like desmin and vimentin could be investigated [85].

Besides the in vitro studies, the dynamics of intermediate filament proteins in living cells could be studied with FCS. 


\title{
Abbreviations and symbols
}

\author{
Abbreviations
}

$\begin{array}{ll}\text { ALS } & \text { amyotrophic lateral sclerosis } \\ \text { ACF } & \text { auto-correlation function } \\ \text { BSA } & \text { bovine serum albumin } \\ \text { CCF } & \text { cross-correlation function } \\ \text { DLS } & \text { dynamic light scattering } \\ \text { FCS } & \text { fluorescence correlation spectroscopy } \\ \text { FCCS } & \text { fluorescence cross-correlation spectroscopy } \\ \text { FEM } & \text { finite element method } \\ \text { IF } & \text { intermediate filament } \\ \text { NDF } & \text { neutral density filter } \\ \text { SPAD } & \text { single-photon avalanche diode } \\ \text { PDMS } & \text { polydimethylsiloxane } \\ \text { ULF } & \text { unit length filament } \\ \text { SW-FCCS } & \text { single wavelength fluorescence cross-correlation spec- } \\ & \text { troscopy }\end{array}$

Symbols

$\begin{array}{ll}c(\vec{r}, t) & \text { concentration } \\ d & \text { diameter of a cylinder } \\ D & \text { diffusion coefficient } \\ D_{20}{ }^{\circ} \mathrm{C}, W & \text { diffusion coefficient at } 20^{\circ} \mathrm{C} \text { in water } \\ f & \text { focal length } \\ F(t) & \text { fluorescent signal }\end{array}$


6 Conclusions and Outlook

$\begin{array}{ll}G(\tau) & \text { correlation function } \\ L & \text { length of a cylinder } \\ n & \text { refractive index } \\ \text { N.A. } & \text { numerical aperture } \\ \mathrm{Pe} & \text { Péclet number } \\ r_{0} & \text { radius of a focused beam } \\ R & \text { radius of the incident beam } \\ \text { Re } & \text { Reynolds number } \\ q & \text { factor describing photophysical properties } \\ T & \text { temperature } \\ T(\tau) & \text { triplet component in correlation function } \\ v & \text { flow velocity } \\ W(\vec{r}) & \text { instrumental detection efficiency } \\ w_{\mathrm{x}, \mathrm{y}} & \text { radius of the detection volume } \\ w_{\mathrm{z}} & \text { elongation of the detection volume } \\ \gamma & \text { structure factor of the detection volume } \\ \eta_{0} & \text { viscosity } \\ \lambda_{\text {exc }} & \text { wavelength of the excitation light } \\ \theta_{\mathrm{T}} & \text { equilibrium population of the triplet state } \\ \rho & \text { fraction of first component } \\ \tau & \text { lag time } \\ \tau_{\mathrm{diff}} & \text { characteristic diffusion time } \\ \tau_{\mathrm{flow}} & \text { characteristic flow time } \\ \tau_{\mathrm{T}} & \text { lifetime of the triplet state } \\ & \end{array}$




\section{A Data acquisition script}

We use the following script for moving the stage and take a FCS measurement at every point. The script was adapted from a sample script for the ALV correlator software. The script automates the data acquisition in ALV correlator software and gives the commands to move the sample stage. The setup acquires FCS curves at every position.

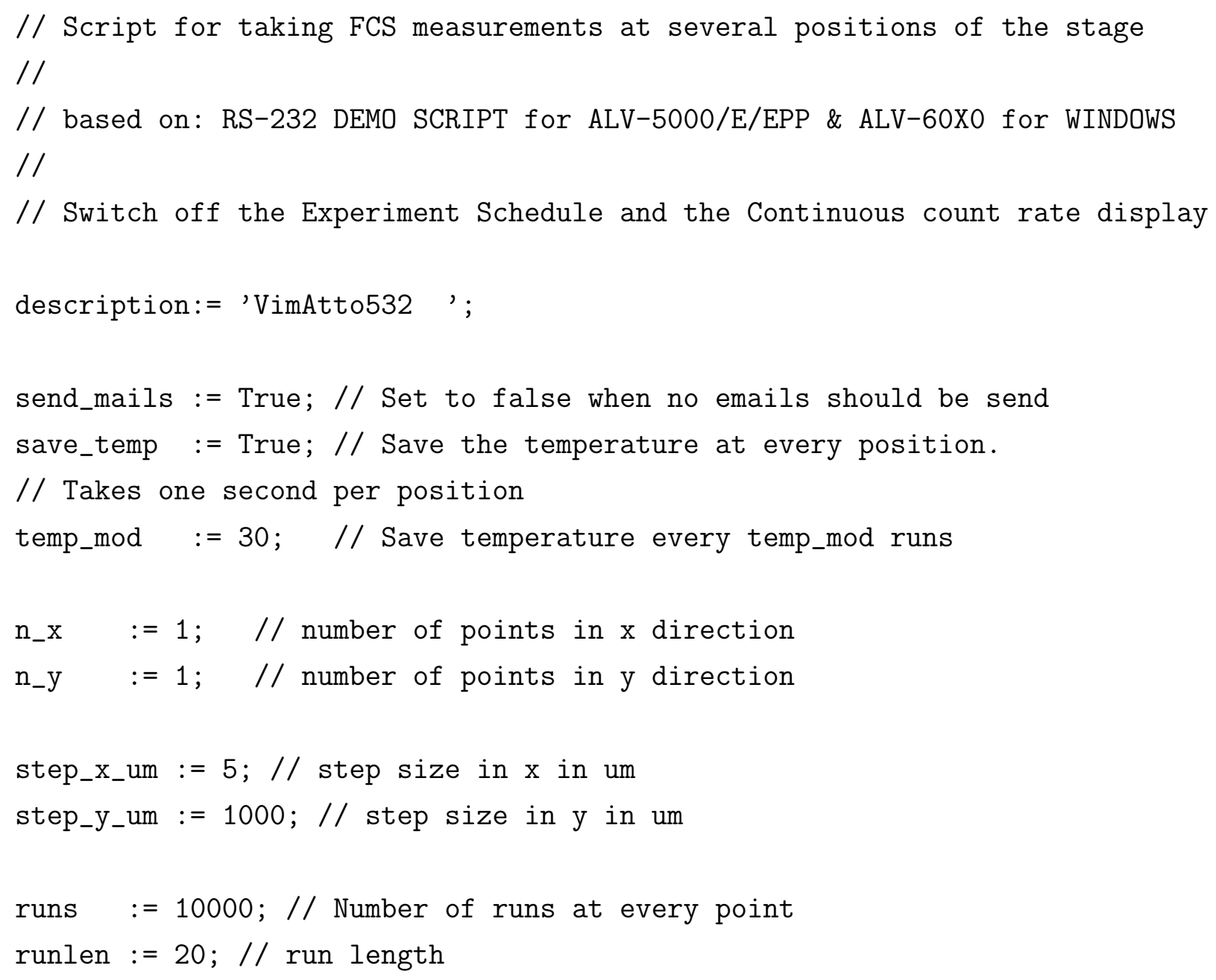


A Data acquisition script

lp $\quad:=20 ; / /$ Laser power in $\mathrm{mW}$

OD $\quad:=2 ; / /$ Optical density of filter

wait_move:=1000; // Time to wait before next stage move in ms wait_disp:=500; // Refresh interval for script display

CorrMode := 2; // CorrMode '2' and 'FCS' in settings

laser_off:= 2000; // Count rate in $\mathrm{kHz}$ at which the laser is switched off

//

// End of config section

//

step_x := step_x_um $* 50 ; / /$ times 50 for ProScan

step_y := step_y_um $* 50 ; / /$ in $\mathrm{nm}$

TextClear;

SetCorrMode (CorrMode);

SetAutoScale (3);

Shell ('taskkill', '/IM PriorTest.exe /F');

wait (100);

baud $:=9600$;

port $:=1$;

Prior := OpenCom(port, baud, 13);

folder:=GetInput ('Set data directory');

folder_mkdir $:=$,"' + folder + '"',

shell_string := '"import os; os.mkdir(") + folder_mkdir + '")"';

Shell ('python -c', shell_string);

mkdir_data $:=$ '"' + folder + '/data"';

shell_string := '"import os; os.mkdir("' + mkdir_data + '")"',

Shell ('python -c', shell_string);

mkdir_qf $:=$ '"' + folder + '/qf"' ; 
shell_string := ' "import os; os.mkdir("' + mkdir_qf + '")"';

Shell ('python -c', shell_string);

mkdir_results $:=$ '" ' + folder + '/results"';

shell_string := '"import os; os.mkdir(" + mkdir_results + '")"';

//Shell('python -c', shell_string);

file := folder+'/data/data'; // numbering and .acs will be added

logfile $:=$ foldert'/log.txt';

runs_file:= folder+'/runs.txt';

$\mathrm{n} \quad:=\left(\mathrm{n}_{-} \mathrm{x}\right) *\left(\mathrm{n}_{-} \mathrm{y}\right) ; / /$ number of positions

if Prior $>0$ then

begin

TextOut('Port to Prior XY Stage opened.');

end

else

begin

TextOut('Could not open port to Prior XY Stage.');

end;

step_str := 'X,' + IntToStr(step_x) + ',' + IntToStr (step_y);

sendCom(Prior, step_str); // Sets the step size $\mathrm{x}, \mathrm{y}$

receiveCom(Prior, 0);

sendCom(Prior, 'X'); // Reports the step size $\mathrm{x}, \mathrm{y}$

TextOut('Step sizes ', receiveCom(Prior, 0));

$\mathrm{nr}$ _steps_str $:={ }^{\prime} \mathrm{N}, '+\operatorname{IntToStr}\left(\mathrm{n}_{-} \mathrm{x}-1\right)+{ }^{\prime},{ }^{\prime}+\operatorname{IntToStr}\left(\mathrm{n}_{-} \mathrm{y}-1\right)$;

sendCom(Prior, nr_steps_str); // Sets the number of $\mathrm{x}$ and $\mathrm{y}$ steps

receiveCom(Prior, 0 );

sendCom(Prior, 'N'); // Reports the number of $\mathrm{x}$ and $\mathrm{y}$ steps 


\section{A Data acquisition script}

textout('Number of steps: ', receiveCom(Prior, 0));

sendCom(Prior, 'E'); // Sets the origin to the current position receiveCom(Prior, 0);

some_variable:=GetInput ('Press Enter');

$\operatorname{logHandle}:=$ OpenTextFile(logfile);

WriteTextFile(logHandle, format ( $\% \mathrm{~s}, \% \mathrm{~s} \% \mathrm{~s}$ ' , 'Folder', folder, \#13\#10)) ;

WriteTextFile (logHandle, format ( $\% \mathrm{~s}, \% 0.13 \mathrm{f} \% \mathrm{~s}$ ', ' Time', now ,\#13\#10));

WriteTextFile (logHandle, format ( $\% \mathrm{~s}, \% \mathrm{~d} \% \mathrm{~s}$ ' , 'lp', lp, \#13\#10));

WriteTextFile (logHandle, format ( $\% \mathrm{~s}, \% \mathrm{~d} \% \mathrm{~s}$ ', 'OD', OD, \#13\#10));

WriteTextFile (logHandle, format ( $\% \mathrm{~s}, \% \mathrm{~d} \% \mathrm{~s}^{\prime}$, ' $\mathrm{n}_{-} \mathrm{x}$ ', $\left.\mathrm{n}_{-} \mathrm{x}, \# 13 \# 10\right)$ );

WriteTextFile (logHandle, format ( $\% \mathrm{~s}, \% \mathrm{~d} \% \mathrm{~s}$ ', ' $\mathrm{n}_{-} \mathrm{y}$ ', $\left.\mathrm{n}_{-} \mathrm{y}, \# 13 \# 10\right)$ );

WriteTextFile (logHandle, format ( $\% \mathrm{~s}, \% \mathrm{~d} \% \mathrm{~s}^{\prime}$, ' 'n', $\left.\mathrm{n}, \# 13 \# 10\right)$ );

WriteTextFile (logHandle,format ( $\%$ s, \% d\%s', 'step_x_um', step_x_um, \#13\#10));

WriteTextFile (logHandle,format ( $\%$ s, \% d\%s' , 'step_y_um', step_y_um, \#13\#10));

WriteTextFile(logHandle, format ( $\% \mathrm{~s}, \% \mathrm{~d} \% \mathrm{~s}$ ', 'runs', runs, \#13\#10));

WriteTextFile (logHandle, format ( $\% \mathrm{~s}, \% \mathrm{~d} \% \mathrm{~s}$ ', 'runlen', runlen, \#13\#10));

WriteTextFile (logHandle, format ( $\% \mathrm{~s}, \% \mathrm{~d} \% \mathrm{~s}$ ', 'CorrMode', CorrMode, \#13\#10));

WriteTextFile (logHandle, format ( $\%$ s, $\%$ s $\% s^{\prime}$ ', 'description', description, \#13\#10));

CloseTextFile(logHandle);

runsHandle $:=$ OpenTextFile(runs_file);

log_str $:=$ format $\left({ }^{\circ} \% \mathrm{~s}, \% \mathrm{~s}, \% \mathrm{~s}, \% \mathrm{~s}, \% \mathrm{~s}, \% \mathrm{~s}, \% \mathrm{~s}, \% \mathrm{~s} \% \mathrm{~s}\right.$ ', 'Pos number', 'pos_x', 'pos_y', 'runs', 'runlength', 'MeanCR0', 'MeanCR1', 'Frac days', \#13\#10);

WriteTextFile(runsHandle, log_str);

for $i:=0$ to $n-1$ do

begin

sendCom(Prior, 'S,?'); // Step number of scan 


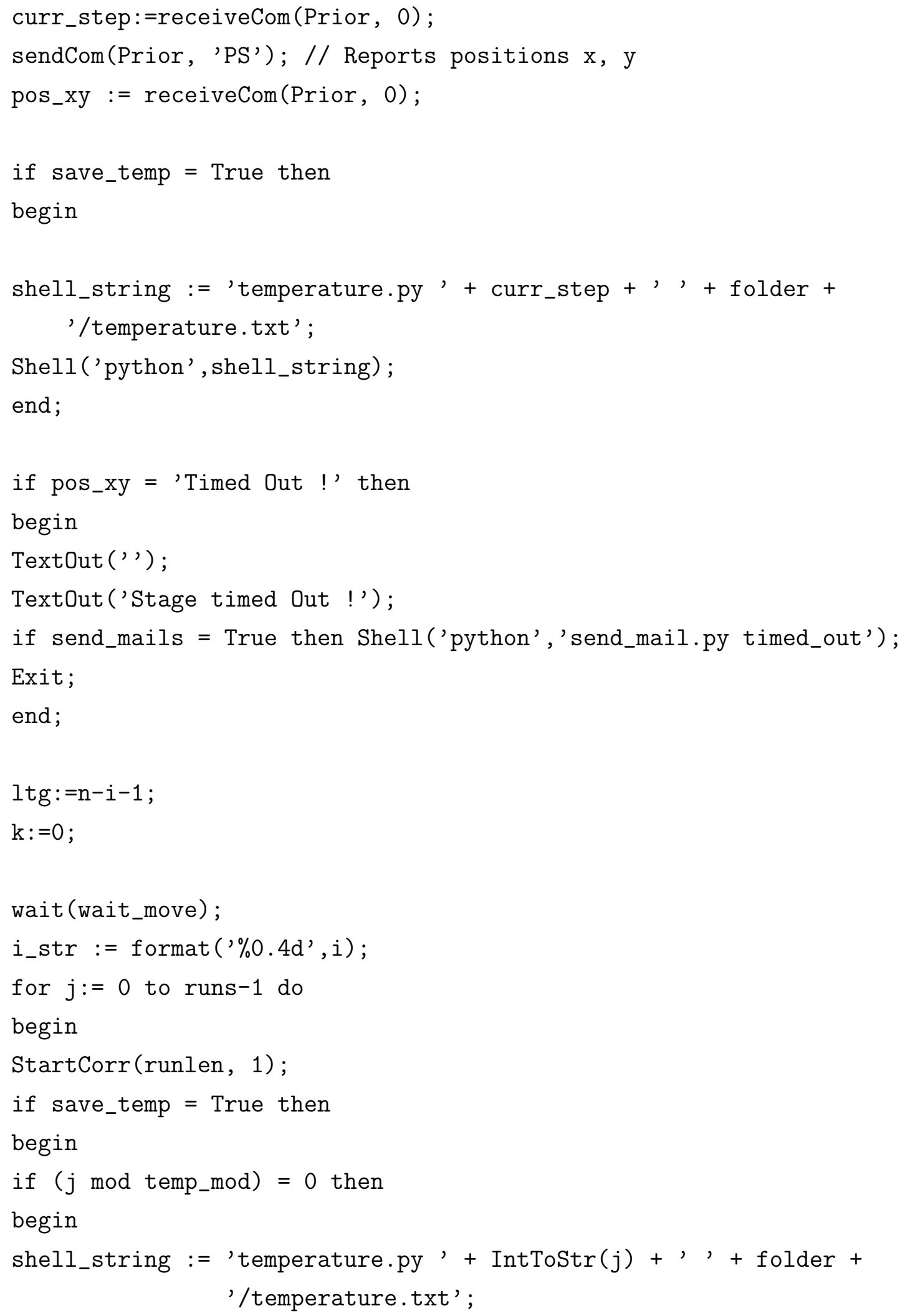


A Data acquisition script

Shell ('python', shell_string);

end ;

end;

while (isRunning $=$ true) do

begin

TextClear;

TextOut ('Position number', i);

TextOut ('Left to go ', Itg);

TextOut ('Step number: ', curr_step);

TextOut('Position: ', pos_xy);

TextOut('Runs left at this pos: ', runs-j);

TextOut (format ('Correlator runs since \%d s', k)) ;

TextOut (format ('Time left in minutes: \%f', ltg*runlen*runs/60)) ;

TextOut (format ('CR0 \%8.3f $\mathrm{kHz}$ ', actualCR0));

TextOut (format ('CR1 \%8.3f kHz ', actualCR1));

if (actualCRO > laser_off) then

begin

Shell ('python', 'C: \send-usb-command.py off1');

TextOut('Too much light! Laser switched off !!!');

Shell ('taskkill', '/IM coboltMonitor.exe /F');

wait (100);

baud $:=115400$;

port := 7 ;

Cobolt := OpenCom (port, baud, 13);

sendCom(Cobolt, '10'); //Shutoff

sn: =receiveCom (Cobolt, 0$)$;

CloseCom(Cobolt);

if send_mails = True then Shell('python', 'send_mail.py failed');

Exit;

end;

if (actualCR1 > laser_off) then

begin

Shell ('python', 'C: \send-usb-command.py off1');

TextOut('Too much light! Laser switched off !!!'); 
Shell ('taskkill', '/IM coboltMonitor.exe /F');

wait (100);

baud := 115400;

port $:=7$;

Cobolt := OpenCom(port, baud, 13);

sendCom(Cobolt, '10'); //Shutoff

sn: $=$ receiveCom (Cobolt, 0$)$;

CloseCom(Cobolt);

if send_mails = True then Shell('python', 'send_mail.py failed');

Exit;

end;

Wait (wait_disp);

$\operatorname{inc}(\mathrm{k})$;

end;

j_str $:=$ format $\left({ }^{\prime} \% 0.2 d^{\prime}, j\right)$;

filename := file + '_pos'+ i_str + '_run' + j_str + '.asc';

SaveData(filename, false, false);

// Standard-Deviation and fitting needs to be set to false

end;

$\log \_s t r:=$ format $\left(\% \% \mathrm{~d}, \% \mathrm{~s}, \% \mathrm{~d}, \% \mathrm{~d}, \% 0.13 \mathrm{f} \% \mathrm{~s}^{\prime}\right.$, i,pos_xy, runs, runlen,now, \#13\#10); WriteTextFile (runsHandle, log_str);

sendCom(Prior, 'S'); // Move to the next position in the Rectangular Raster receiveCom(Prior, 0);

end;

CloseTextFile (runsHandle);

TextOut (' ');

TextOut ('Measurement Done !');

CloseCom(Prior);

if send_mails = True then Shell('python', 'send_mail.py successful'); 
A Data acquisition script 


\section{Bibliography}

[1] M. Hesse, T. M. Magin, and K. Weber. "Genes for Intermediate Filament Proteins and the Draft Sequence of the Human Genome". In: Journal of Cell Science 114.14 (July 2001), pp. 2569-2575 (cit. on p. 1).

[2] M. B. Omary. " "IF-Pathies": A Broad Spectrum of Intermediate Filament-associated Diseases". In: Journal of Clinical Investigation 119.7 (July 2009), pp. 1756-1762. DOI: $10.1172 /$ JCI39894 (cit. on pp. 1, 4).

[3] F. Huber, A. Boire, M. P. López, and G. H. Koenderink. "Cytoskeletal Crosstalk: When Three Different Personalities Team Up". In: Current Opinion in Cell Biology. Cell architecture 32 (Feb. 2015), pp. 39-47. DOI: 10.1016/j . ceb.2014.10. 005 (cit. on p. 3).

[4] H. Herrmann, H. Bär, L. Kreplak, S. V. Strelkov, and U. Aebi. "Intermediate Filaments: From Cell Architecture to Nanomechanics". In: Nature Reviews Molecular Cell Biology 8.7 (Jan. 2007), pp. 562-573. DOr: 10.1038/nrm2197 (cit. on p. 3).

[5] T. Lichtenstern, N. Mücke, U. Aebi, M. Mauermann, and H. Herrmann. "Complex Formation and Kinetics of Filament Assembly Exhibited by the Simple Epithelial Keratins K8 and K18". In: Journal of Structural Biology 177.1 (Jan. 2012), pp. 5462. DOI: $10.1016 / \mathrm{j} \cdot \mathrm{jsb} .2011 .11 .003$ (cit. on p. 3).

[6] H. Herrmann, M. Häner, M. Brettel, N.-O. Ku, and U. Aebi. "Characterization of Distinct Early Assembly Units of Different Intermediate Filament Proteins". In: Journal of Molecular Biology 286.5 (Mar. 1999), pp. 1403-1420. DOI: 10.1006/ jmbi.1999.2528 (cit. on p. 3).

[7] M. Müller, S. S. Bhattacharya, T. Moore, Q. Prescott, T. Wedig, H. Herrmann, and T. M. Magin. "Dominant Cataract Formation in Association with a Vimentin Assembly Disrupting Mutation". In: Human Molecular Genetics 18.6 (Mar. 2009), pp. 1052-1057. DOI: $10.1093 / \mathrm{hmg} / \mathrm{ddn} 440$ (cit. on p. 4). 
Bibliography

[8] E Fuchs and K Weber. "Intermediate Filaments: Structure, Dynamics, Function and Disease". In: Annual Review of Biochemistry 63.1 (1994), pp. 345-382. DOI: 10.1146/annurev.bi.63.070194.002021 (cit. on p. 4).

[9] H. Herrmann, M. Häner, M. Brettel, S. A. Müller, K. N. Goldie, B. Fedtke, A. Lustig, W. W. Franke, and U. Aebi. "Structure and Assembly Properties of the Intermediate Filament Protein Vimentin: The Role of Its Head, Rod and Tail Domains". In: Journal of Molecular Biology 264.5 (Dec. 1996), pp. 933-953. DOI: 10.1006/jmbi.1996.0688 (cit. on pp. 5, 6).

[10] G. Çolakoğlu and A. Brown. "Intermediate Filaments Exchange Subunits along Their Length and Elongate by End-to-End Annealing". In: The Journal of Cell Biology 185.5 (Jan. 2009), pp. 769-777. DOI: 10.1083/ jcb. 200809166 (cit. on p. 5).

[11] B. Nöding, H. Herrmann, and S. Köster. "Direct Observation of Subunit Exchange along Mature Vimentin Intermediate Filaments". In: Biophysical Journal 107.12 (Dec. 2014), pp. 2923-2931. DOI: 10.1016/j.bpj.2014.09.050 (cit. on pp. 5, 6).

[12] P. M. Steinert, L. N. Marekov, and D. A. Parry. "Diversity of Intermediate Filament Structure. Evidence That the Alignment of Coiled-Coil Molecules in Vimentin Is Different from That in Keratin Intermediate Filaments." In: Journal of Biological Chemistry 268.33 (Nov. 1993), pp. 24916-24925 (cit. on p. 5).

[13] A. A. Chernyatina, D. Guzenko, and S. V. Strelkov. "Intermediate Filament Structure: The Bottom-up Approach". In: Current Opinion in Cell Biology. Cell architecture 32 (Feb. 2015), pp. 65-72. DOI: 10.1016/ j. ceb.2014.12.007 (cit. on p. 5 ).

[14] I Hofmann, H Herrmann, and W Franke. "Assembly and Structure of CalciumInduced Thick Vimentin Filaments." In: European journal of cell biology 56.2 (1991/12, 1991), pp. 328-341 (cit. on p. 5).

[15] M. Kooijman, M. Bloemendal, P. Traub, R. van Grondelle, and H. van Amerongen. "Transient Electric Birefringence Study of Intermediate Filament Formation from Vimentin and Glial Fibrillary Acidic Protein". In: Journal of Biological Chemistry 272.36 (May 1997), pp. 22548-22555. DOI: 10.1074/jbc.272.36.22548 (cit. on p. 5). 
[16] H. Herrmann and U. Aebi. "Intermediate Filament Assembly: Temperature Sensitivity and Polymorphism". In: Cellular and Molecular Life Sciences CMLS 55.11 (Aug. 1999), pp. 1416-1431. DOI: 10.1007/s000180050382 (cit. on p. 5).

[17] S. Winheim, A. R. Hieb, M. Silbermann, E.-M. Surmann, T. Wedig, H. Herrmann, J. Langowski, and N. Mücke. "Deconstructing the Late Phase of Vimentin Assembly by Total Internal Reflection Fluorescence Microscopy (TIRFM)". In: PLoS ONE 6.4 (Apr. 2011), e19202. DOI: 10.1371/ journal . pone.0019202 (cit. on pp. 6, 19, 70,

[18] M. Meier, G. P. Padilla, H. Herrmann, T. Wedig, M. Hergt, T. R. Patel, J. Stetefeld, U. Aebi, and P. Burkhard. "Vimentin Coil 1A-A Molecular Switch Involved in the Initiation of Filament Elongation". In: Journal of Molecular Biology 390.2 (July 2009), pp. 245-261. DOI: 10.1016/j.jmb.2009.04.067 (cit. on p. 6).

[19] A. V. Sokolova, L. Kreplak, T. Wedig, N. Mücke, D. I. Svergun, H. Herrmann, U. Aebi, and S. V. Strelkov. "Monitoring Intermediate Filament Assembly by Small-Angle x-Ray Scattering Reveals the Molecular Architecture of Assembly Intermediates". In: Proceedings of the National Academy of Sciences 103.44 (Oct. 2006), pp. 16206-16211. DOI: 10.1073/pnas.0603629103 (cit. on p. 6).

[20] M. E. Brennich, J.-F. Nolting, C. Dammann, B. Nöding, S. Bauch, H. Herrmann, T. Pfohl, and S. Köster. "Dynamics of Intermediate Filament Assembly Followed in Micro-Flow by Small Angle X-Ray Scattering". In: Lab on a Chip 11.4 (Jan. 2011), pp. 708-716. DOI: 10.1039/COLC00319K (cit. on pp. 6, 9, 56, 71).

[21] N. Mücke, R. Kirmse, T. Wedig, J. Leterrier, and L. Kreplak. "Investigation of the Morphology of Intermediate Filaments Adsorbed to Different Solid Supports". In: Journal of Structural Biology 150.3 (June 2005), pp. 268-276. DOI: 10.1016/j . jsb.2005.02.012 (cit. on pp. 6, 70).

[22] C. G. Lopez, O. Saldanha, K. Huber, and S. Köster. "Lateral Association and Elongation of Vimentin Intermediate Filament Proteins: A Time-Resolved LightScattering Study". In: Proceedings of the National Academy of Sciences 113.40 (Apr. 2016), pp. 11152-11157. DOI: 10.1073/pnas. 1606372113 (cit. on pp. 6. 70 . 
Bibliography

[23] S. Portet, N. Mücke, R. Kirmse, J. Langowski, M. Beil, and H. Herrmann. "Vimentin Intermediate Filament Formation: In Vitro Measurement and Mathematical Modeling of the Filament Length Distribution during Assembly†". In: Langmuir 25.15 (Aug. 2009), pp. 8817-8823. DOI: 10.1021/la900509r (cit. on pp. 6. 70.).

[24] N. Mücke, L. Kreplak, R. Kirmse, T. Wedig, H. Herrmann, U. Aebi, and J. Langowski. "Assessing the Flexibility of Intermediate Filaments by Atomic Force Microscopy". In: Journal of Molecular Biology 335.5 (Jan. 2004), pp. 1241-1250. DOI: $10.1016 / j \cdot j \mathrm{mb} .2003 .11 .038$ (cit. on p. 6).

[25] B. Nöding and S. Köster. "Intermediate Filaments in Small Configuration Spaces". In: Physical Review Letters 108.8 (Feb. 2012), p. 088101. DOI: 10.1103/PhysRevLett . 108.088101 (cit. on p. 6).

[26] J. Block, H. Witt, A. Candelli, E. J. G. Peterman, G. J. L. Wuite, A. Janshoff, and S. Köster. "Nonlinear Loading-Rate-Dependent Force Response of Individual Vimentin Intermediate Filaments to Applied Strain". In: Physical Review Letters 118.4 (Jan. 2017), p. 048101. DOI: 10.1103/PhysRevLett.118.048101 (cit. on p. 6).

[27] D. D. Carlo. "Inertial Microfluidics". In: Lab on a Chip 9.21 (Nov. 2009), pp. 30383046. DOI: $10.1039 /$ B912547G (cit. on p. 7).

[28] T. M. Squires and S. R. Quake. "Microfluidics: Fluid Physics at the Nanoliter Scale". In: Reviews of Modern Physics 77.3 (Oct. 2005), pp. 977-1026. DOI: 10 . 1103/RevModPhys.77.977 (cit. on p. 8).

[29] A. S. Kane, A. Hoffmann, P. Baumgärtel, R. Seckler, G. Reichardt, D. A. Horsley, B. Schuler, and O. Bakajin. "Microfluidic Mixers for the Investigation of Rapid Protein Folding Kinetics Using Synchrotron Radiation Circular Dichroism Spectroscopy". In: Analytical Chemistry 80.24 (Dec. 2008), pp. 9534-9541. DOI: 10 . 1021/ac801764r (cit. on p. 9).

[30] H. Y. Park, S. A. Kim, J. Korlach, E. Rhoades, L. W. Kwok, W. R. Zipfel, M. N. Waxham, W. W. Webb, and L. Pollack. "Conformational Changes of Calmodulin upon Ca2+ Binding Studied with a Microfluidic Mixer". In: Proceedings of the National Academy of Sciences of the United States of America 105.2 (Jan. 2008), pp. 542-547. DOI: 10.1073/pnas.0710810105 (cit. on p. 9). 
[31] J. B. Knight, A. Vishwanath, J. P. Brody, and R. H. Austin. "Hydrodynamic Focusing on a Silicon Chip: Mixing Nanoliters in Microseconds". In: Physical Review Letters 80.17 (Apr. 1998), pp. 3863-3866. DOI: 10.1103/PhysRevLett.80.3863 (cit. on pp. 9, 51).

[32] B. Ivorra, D. E. Hertzog, B. Mohammadi, and J. G. Santiago. "Semi-Deterministic and Genetic Algorithms for Global Optimization of Microfluidic Protein-Folding Devices". In: International Journal for Numerical Methods in Engineering 66.2 (2006), pp. 319-333. DOI: 10.1002/nme.1562 (cit. on p. 9).

[33] S. H. Pfeil, C. E. Wickersham, A. Hoffmann, and E. A. Lipman. "A Microfluidic Mixing System for Single-Molecule Measurements". In: Review of Scientific Instruments 80.5 (May 2009), pp. 055105-055105-9. DOI: 10.1063/1.3125643 (cit. on pp. 9, 51,53).

[34] B. Wunderlich, D. Nettels, S. Benke, J. Clark, S. Weidner, H. Hofmann, S. H. Pfeil, and B. Schuler. "Microfluidic Mixer Designed for Performing Single-Molecule Kinetics with Confocal Detection on Timescales from Milliseconds to Minutes". In: Nature Protocols 8.8 (Aug. 2013), pp. 1459-1474. DOI: 10.1038/nprot.2013.082 (cit. on pp. 9, 51).

[35] H. Y. Park, X. Qiu, E. Rhoades, J. Korlach, L. W. Kwok, W. R. Zipfel, W. W. Webb, and L. Pollack. "Achieving Uniform Mixing in a Microfluidic Device: Hydrodynamic Focusing Prior to Mixing". In: Analytical Chemistry 78.13 (July 2006), pp. 4465-4473. DOI: 10.1021/ac060572n (cit. on pp. 9, 52).

[36] M. E. Kinahan, E. Filippidi, S. Köster, X. Hu, H. M. Evans, T. Pfohl, D. L. Kaplan, and J. Wong. "Tunable Silk: Using Microfluidics to Fabricate Silk Fibers with Controllable Properties". In: Biomacromolecules 12.5 (May 2011), pp. 15041511. DOI: $10.1021 / \mathrm{bm} 1014624$ (cit. on p. 9).

[37] R. Russell, I. S. Millett, M. W. Tate, L. W. Kwok, B. Nakatani, et al. "Rapid Compaction during RNA Folding". In: Proceedings of the National Academy of Sciences 99.7 (Feb. 2002), pp. 4266-4271. DOI: 10.1073/pnas.072589599 (cit. on p. 9).

[38] O. Saldanha, M. E. Brennich, M. Burghammer, H. Herrmann, and S. Köster. "The Filament Forming Reactions of Vimentin Tetramers Studied in a Serial-Inlet 
Bibliography

Microflow Device by Small Angle x-Ray Scattering". In: Biomicrofluidics 10.2 (Mar. 2016), p. 024108. DOI: 10.1063/1.4943916 (cit. on p. 9).

[39] A. D. Stroock, S. K. W. Dertinger, A. Ajdari, I. Mezić, H. A. Stone, and G. M. Whitesides. "Chaotic Mixer for Microchannels". In: Science 295.5555 (Jan. 2002), pp. 647-651. DOI: $10.1126 /$ science. 1066238 (cit. on p. 10).

[40] A. D. Stroock and G. J. McGraw. "Investigation of the Staggered Herringbone Mixer with a Simple Analytical Model". In: Philosophical Transactions of the Royal Society of London. Series A: Mathematical, Physical and Engineering Sciences 362.1818 (May 2004), pp. 971-986. DOI: 10.1098/rsta.2003.1357 (cit. on p. 10 ).

[41] M. S. Williams, K. J. Longmuir, and P. Yager. "A Practical Guide to the Staggered Herringbone Mixer". In: Lab on a Chip 8.7 (June 2008), pp. 1121-1129. DOI: 10.1039/B802562B (cit. on p. 10).

[42] M. M. Tirado and J. G. de la Torre. "Translational Friction Coefficients of Rigid, Symmetric Top Macromolecules. Application to Circular Cylinders". In: The Journal of Chemical Physics 71.6 (Sept. 1979), pp. 2581-2587. DOI: 10.1063/1.438613 (cit. on p. 10).

[43] M. M. Tirado, C. L. Martínez, and J. G. de la Torre. "Comparison of Theories for the Translational and Rotational Diffusion Coefficients of Rod-like Macromolecules. Application to Short DNA Fragments". In: The Journal of Chemical Physics 81.4 (Aug. 1984), pp. 2047-2052. DOI: doi:10.1063/1.447827 (cit. on p. 10 .

[44] J. G. De La Torre, M. C. L. Martinez, and M. M. Tirado. "Dimensions of Short, Rodlike Macromolecules from Translational and Rotational Diffusion Coefficients. Study of the Gramicidin Dimer". In: Biopolymers 23.4 (Apr. 1984), pp. 611-615. DOI: 10.1002/bip.360230402 (cit. on p. 10).

[45] D. Magde, E. Elson, and W. W. Webb. "Thermodynamic Fluctuations in a Reacting System-Measurement by Fluorescence Correlation Spectroscopy". In: Physical Review Letters 29.11 (Sept. 1972), pp. 705-708. DOI: 10.1103/PhysRevLett. 29.705 (cit. on p. 11).

[46] E. L. Elson and D. Magde. "Fluorescence Correlation Spectroscopy. I. Conceptual Basis and Theory". In: Biopolymers 13.1 (Jan. 1974), pp. 1-27. DOI: 10.1002/ bip.1974.360130102 (cit. on p. 11). 
[47] D. Magde, E. L. Elson, and W. W. Webb. "Fluorescence Correlation Spectroscopy. II. An Experimental Realization". In: Biopolymers 13.1 (1974), pp. 29-61. DOI: 10.1002/bip.1974.360130103 (cit. on p. 11).

[48] D. Magde, W. W. Webb, and E. L. Elson. "Fluorescence Correlation Spectroscopy. III. Uniform Translation and Laminar Flow". In: Biopolymers 17.2 (1978), pp. 361376. DOI: 10.1002/bip.1978.360170208 (cit. on pp. 11, 15).

[49] T. Weidemann, J. Mücksch, and P. Schwille. "Fluorescence Fluctuation Microscopy: A Diversified Arsenal of Methods to Investigate Molecular Dynamics inside Cells". In: Current Opinion in Structural Biology 28 (Oct. 2014), pp. 69-76. DOI: 10.1016/ j.sbi.2014.07.008 (cit. on p. 12).

[50] A. Gennerich. "Fluoreszenzkorrelationsspektroskopie und Rasterkorrelationsmikroskopie molekularer Prozesse in Nervenzellen". PhD thesis. Nov. 2003. (Accessed on 01/17/2014) (cit. on p. 12).

[51] P. Müller, P. Schwille, and T. Weidemann. "PyCorrFit-generic Data Evaluation for Fluorescence Correlation Spectroscopy". In: Bioinformatics 30.17 (Jan. 2014), pp. 2532-2533. DOI: 10.1093/bioinformatics/btu328 (cit. on p. 13).

[52] C. B. Müller, A. Loman, V. Pacheco, F. Koberling, D. Willbold, W. Richtering, and J. Enderlein. "Precise Measurement of Diffusion by Multi-Color Dual-Focus Fluorescence Correlation Spectroscopy". In: EPL (Europhysics Letters) 83.4 (Aug. 2008), p. 46001. DOI: 10.1209/0295-5075/83/46001 (cit. on pp. 15, 36).

[53] Z. Petrášek and P. Schwille. "Precise Measurement of Diffusion Coefficients Using Scanning Fluorescence Correlation Spectroscopy". In: Biophysical Journal 94.4 (Feb. 2008), pp. 1437-1448. DOI: 10.1529/biophysj.107.108811 (cit. on pp. 15 , 17).

[54] M. Gösch, H. Blom, J. Holm, T. Heino, and R. Rigler. "Hydrodynamic Flow Profiling in Microchannel Structures by Single Molecule Fluorescence Correlation Spectroscopy". In: Analytical Chemistry 72.14 (July 2000), pp. 3260-3265. DOI: 10.1021/ac991448p (cit. on p. 15).

[55] T. J. Arbour and J. Enderlein. "Application of Dual-Focus Fluorescence Correlation Spectroscopy to Microfluidic Flow-Velocity Measurement". In: Lab on a Chip 10.10 (May 2010), pp. 1286-1292. DOI: 10.1039/B924594D (cit. on pp. 15, 69). 
Bibliography

[56] J. Widengren, R. Rigler, and Ü. Mets. "Triplet-State Monitoring by Fluorescence Correlation Spectroscopy". In: Journal of Fluorescence 4.3 (Sept. 1994), pp. 255258. DOI: $10.1007 / \mathrm{BF} 01878460$ (cit. on p. 16).

[57] M. Zhao, L. Jin, B. Chen, Y. Ding, H. Ma, and D. Chen. "Afterpulsing and Its Correction in Fluorescence Correlation Spectroscopy Experiments". In: Applied Optics 42.19 (July 2003), pp. 4031-4036. DOI: 10.1364/A0.42.004031 (cit. on p. 16.

[58] P. Schwille, F. Meyer-Almes, and R. Rigler. "Dual-Color Fluorescence CrossCorrelation Spectroscopy for Multicomponent Diffusional Analysis in Solution". In: Biophysical Journal 72.4 (Apr. 1997), pp. 1878-1886. DOI: 10.1016/S00063495(97) 78833-7 (cit. on p. 16).

[59] L. C. Hwang and T. Wohland. "Dual-Color Fluorescence Cross-Correlation Spectroscopy Using Single Laser Wavelength Excitation". In: ChemPhysChem 5.4 (2004), pp. 549-551. DOI: 10.1002/cphc. 200301057 (cit. on pp. 16, 33).

[60] M. Brinkmeier, K. Dörre, J. Stephan, and M. Eigen. "Two-Beam Cross-Correlation: A Method To Characterize Transport Phenomena in Micrometer-Sized Structures". In: Analytical Chemistry 71.3 (Feb. 1999), pp. 609-616. DOI: 10.1021/ ac980820i (cit. on pp. 16, 54, 69.).

[61] T. Dertinger, V. Pacheco, I. von der Hocht, R. Hartmann, I. Gregor, and J. Enderlein. "Two-Focus Fluorescence Correlation Spectroscopy: A New Tool for Accurate and Absolute Diffusion Measurements". In: ChemPhysChem 8.3 (2007), pp. 433443. DOI: $10.1002 / \mathrm{cphc} .200600638$ (cit. on p. 16).

[62] K. M. Berland, P. T. So, Y. Chen, W. W. Mantulin, and E. Gratton. "Scanning Two-Photon Fluctuation Correlation Spectroscopy: Particle Counting Measurements for Detection of Molecular Aggregation". In: Biophysical Journal 71.1 (July 1996), pp. 410-420. DOI: 10.1016/S0006-3495(96)79242-1 (cit. on p. 17).

[63] H. Herrmann, L. Kreplak, and U. Aebi. "Isolation, Characterization, and In Vitro Assembly of Intermediate Filaments". In: Methods in Cell Biology. Ed. by M. B. Omary and P. A. Coulombe. Vol. Volume 78. Intermediate Filament Cytoskeleton. Academic Press, 2004, pp. 3-24. ISBN: 0091-679X. (Accessed on 09/09/2014) (cit. on p. 19). 
[64] H. S. Harned and R. L. Nuttall. "The Diffusion Coefficient of Potassium Chloride in Dilute Aqueous Solution". In: Journal of the American Chemical Society 69.4 (Apr. 1947), pp. 736-740. DOI: 10.1021/ja01196a002 (cit. on p. 21).

[65] D. C. Duffy, J. C. McDonald, O. J. A. Schueller, and G. M. Whitesides. "Rapid Prototyping of Microfluidic Systems in Poly(Dimethylsiloxane)". In: Analytical Chemistry 70.23 (Dec. 1998), pp. 4974-4984. DOI: 10.1021/ac980656z (cit. on p. 23).

[66] E. Kim, Y. Xia, and G. M. Whitesides. "Micromolding in Capillaries: Applications in Materials Science". In: Journal of the American Chemical Society 118.24 (June 1996). DOI: $10.1021 /$ ja960151v (cit. on p. 23).

[67] M. Krogh. My Little Guide to Soft Lithography. http://www . ifm.liu.se/ applphys/biorgel/education/mikrosystem-nanobiologi-t/material-samttipstricks/Soft_Lithography_for_Dummies.pdf. 2003. (Accessed on 10/25/2016) (cit. on p. 24).

[68] A. Edelstein, N. Amodaj, K. Hoover, R. Vale, and N. Stuurman. "Computer Control of Microscopes Using Micro-Manager". In: Current Protocols in Molecular Biology (2010). DOI: 10.1002/0471142727.mb1420s92 (cit. on p. 28 ).

[69] ThermoFisher Scientific. AlexaFluor 532 Spectra. https://www . thermofisher. $\mathrm{com} / \mathrm{de} / \mathrm{de} / \mathrm{home} / \mathrm{life}$ - science / cell - analysis / labeling - chemistry / fluorescence-spectraviewer.html. (Accessed on 10/24/2016) (cit. on p. 28).

[70] J. Schindelin, I. Arganda-Carreras, E. Frise, V. Kaynig, M. Longair, et al. "Fiji: An Open-Source Platform for Biological-Image Analysis". In: Nature Methods 9.7 (July 2012), pp. 676-682. DOI: 10.1038/nmeth.2019 (cit. on p. 30).

[71] ATTO-TEC GmbH. ATTO 532 Spectra.https://www.atto-tec.com/attotecshop/ product_info.php?info=p102_atto-532.html. (Accessed on 01/26/2017) (cit. on p. 33).

[72] AHF analysentechnik AG. Laser Beamsplitter Zt 532 RDC. https://www . ahf . de / art-Laser-Strahlenteiler_zt_532_RDC; F48-533 . html. (Accessed on 10/24/2016) (cit. on p. 33).

[73] AHF analysentechnik AG. RazorEdge LP Edge Filter 532 RU. https: //www . ahf.de/art-RazorEdge_LP_Edge_Filter_532_RU;F76-532.html. (Accessed on 01/26/2017) (cit. on p. 33). 
Bibliography

[74] Pierre Cladé. PyDAQmx : A Python Interface to the National Instruments DAQmx Driver. http://pythonhosted.org/PyDAQmx/. (Accessed on 11/13/2016) (cit. on p. 34).

[75] J. W. Krieger and J. Langowski. QuickFit 3.0 (Compiled: 2015-10-29, SVN: 4465): A Data Evaluation Application for Biophysics. 2010-2016 (cit. on p. 34).

[76] K. Nakanishi, T. Sakiyama, and K. Imamura. "On the Adsorption of Proteins on Solid Surfaces, a Common but Very Complicated Phenomenon". In: Journal of Bioscience and Bioengineering 91.3 (2001), pp. 233-244. DOI: 10.1016/S13891723(01)80127-4 (cit. on p. 52).

[77] H. Makamba, J. H. Kim, K. Lim, N. Park, and J. H. Hahn. "Surface Modification of Poly(Dimethylsiloxane) Microchannels". In: ELECTROPHORESIS 24.21 (Nov. 2003), pp. 3607-3619. DOI: 10.1002/elps.200305627 (cit. on p. 52 ).

[78] S. Nath, J. Meuvis, J. Hendrix, S. A. Carl, and Y. Engelborghs. "Early Aggregation Steps in $\alpha$-Synuclein as Measured by FCS and FRET: Evidence for a Contagious Conformational Change". In: Biophysical Journal 98.7 (Apr. 2010), pp. 1302-1311. DOI: $10.1016 / \mathrm{j} . \mathrm{bpj} .2009 .12 .4290$ (cit. on p. 70).

[79] T. Krouglova, J. Vercammen, and Y. Engelborghs. "Correct Diffusion Coefficients of Proteins in Fluorescence Correlation Spectroscopy. Application to Tubulin Oligomers Induced by Mg2+ and Paclitaxel". In: Biophysical Journal 87.4 (Oct. 2004), pp. 2635-2646. DOI: 10.1529/biophysj.104.040717 (cit. on p. 70).

[80] P. Sengupta, K. Garai, J. Balaji, N. Periasamy, and S. Maiti. "Measuring Size Distribution in Highly Heterogeneous Systems with Fluorescence Correlation Spectroscopy". In: Biophysical Journal 84.3 (Mar. 2003), pp. 1977-1984. DOI: 10.1016/ S0006-3495(03)75006-1 (cit. on p. 70).

[81] K. Módos, R. Galántai, I. Bárdos-Nagy, M. Wachsmuth, K. Tóth, J. Fidy, and J. Langowski. "Maximum-Entropy Decomposition of Fluorescence Correlation Spectroscopy Data: Application to Liposome-human Serum Albumin Association". In: European Biophysics Journal 33.1 (Feb. 2004), pp. 59-67. DOI: 10.1007/s00249003-0343-6 (cit. on p. 70). 
[82] R. Kirmse, S. Portet, N. Mücke, U. Aebi, H. Herrmann, and J. Langowski. "A Quantitative Kinetic Model for the in Vitro Assembly of Intermediate Filaments from Tetrameric Vimentin". In: Journal of Biological Chemistry 282.25 (June 2007), pp. 18563-18572. DOI: 10.1074/jbc.M701063200 (cit. on p. 70.

[83] N. Mücke, S. Winheim, H. Merlitz, J. Buchholz, J. Langowski, and H. Herrmann. "In Vitro Assembly Kinetics of Cytoplasmic Intermediate Filaments: A Correlative Monte Carlo Simulation Study". In: PLOS ONE 11.6 (June 2016), e0157451. DOI: 10.1371/journal.pone.0157451 (cit. on p. 70).

[84] S. Rüttinger, V. Buschmann, B. Krämer, R. Erdmann, R. Macdonald, and F. Koberling. "Comparison and Accuracy of Methods to Determine the Confocal Volume for Quantitative Fluorescence Correlation Spectroscopy". In: Journal of Microscopy 232.2 (2008), pp. 343-352. DOI: 10.1111/j.1365-2818.2008.02105.x (cit. on p. 73).

[85] U. Wickert, N. Mücke, T. Wedig, S. A. Müller, U. Aebi, and H. Herrmann. "Characterization of the in Vitro Co-Assembly Process of the Intermediate Filament Proteins Vimentin and Desmin: Mixed Polymers at All Stages of Assembly". In: European Journal of Cell Biology 84.2-3 (Mar. 2005), pp. 379-391. DOI: 10.1016/ j.ejcb.2005.01.004 (cit. on p. 74). 
Bibliography 


\section{List of publications}

Johanna Block, Viktor Schroeder, Paul Pawelzyk, Norbert Willenbacher, and Sarah Köster. "Physical Properties of Cytoplasmic Intermediate Filaments." Biochimica et Biophysica Acta (BBA) - Molecular Cell Research, Mechanobiology, 1853, no. 11, Part B (November 2015): 3053-64. doi:10.1016/j.bbamcr.2015.05.009. 
Bibliography 


\section{Danksagung}

Ich möchte an dieser Stelle bei allen bedanken, die mich bei der Anfertigung dieser Arbeit unterstützt haben.

Sarah Köster möchte ich dafür danken, dass ich diese Arbeit in ihrer Arbeitsgruppe anfertigen konnte. Ihre umfassende Betreuung war sehr wichtig für das Gelingen dieser Arbeit.

Florian Rehfeldt möchte ich für sein Engagement in meinem Betreuungsausschuss und die Übernahme des Koreferats dieser Dissertation danken. Desweiteren möchte ich mich für die Einführung in die AFM-Messungen bedanken.

Detlev Schild danke ich für sein Engagement in meinem Betreuungsausschuss.

Helmut Grubmüller und Jörg Enderlein möchte ich dafür danken, dass sie an meinem Prüfungskommittee teilgenommen haben.

Harald Herrmann und Norbert Mücke danke ich für die vielen Diskussionen über Intermediärfilamente und das Interesse an meiner Arbeit.

Stefan Winheim und Tanja Tourgaidis möchte ich für die Einführung zum Fluoreszenzmarkieren von Intermediärfilamenten in Heidelberg danken.

Bernd Nöding danke ich für die Vorarbeit und das Zusammenarbeiten beim Aufbau des FCS-Setups.

Anja Niedermeyer, Alexander Brinkmann, Mihai Alevra und Jan Krieger möchte ich für die Unterstützung beim FCS-Aufbau danken.

I want to thank Narain Karedla for the discussions on FCS.

Bei Jochen Herbst möchte ich mich für die Hilfe bei vielen technischen Fragen und die Unterstützung im Reinraum bedanken.

Jan Goemann gilt mein Dank für die Hilfe bei IT-Fragen.

Meinen Kollegen im Büro und im Labor danke ich für die vielen Gespräche und die gute Arbeitsatmosphäre. 


\section{Bibliography}

Meinen Eltern, meiner Schwester und der Familie Kostka danke ich für die Unterstützung während der gesamten Zeit. Meiner Freundin Ines danke ich von ganzem Herzen für die liebevolle Unterstützung. 


\section{Lebenslauf}

Viktor Rudolf Schroeder

geboren am 06. November 1988 in Rinteln

\section{Schulischer und wissenschaftlicher Werdegang}

seit $01 / 2013$

$07 / 2012-12 / 2012$

$10 / 2006-07 / 2012$

$07 / 2006$
Promotionsstudium und wissenschaftlicher Mitarbeiter an der Georg-August-Universität Gschöttingen.

Wissenschaftlicher Mitarbeiter am B CUBE Dresden.

Diplomstudiengang Physik an der Technischen Universität Dresden.

Erlangung der allgemeinen Hochschulreife am Gymnasium Carolinum, Neustrelitz. 